\title{
High-K materials and Metal Gates for CMOS applications
}

\author{
John Robertson \\ Engineering Department, Cambridge University, Cambridge CB2 1PZ, UK
}

Robert M. Wallace

Materials Science and Engineering, University of Texas at Dallas, Richardson, TX 75080, USA

\begin{abstract}
The scaling of complementary metal oxide semiconductor (CMOS) transistors has led to the silicon dioxide layer used as a gate dielectric becoming so thin that the gate leakage current becomes too large. This led to the replacement of $\mathrm{SiO}_{2}$ by a physically thicker layer of a higher dielectric constant or 'high$\mathrm{K}$ ' oxide such as hafnium oxide. Intensive research was carried out to develop these oxides into high quality electronic materials. In addition, the incorporation of Ge in the CMOS transistor structure has been employed to enable higher carrier mobility and performance. This review covers both scientific and technological issues related to the high-K gate stack - the choice of oxides, their deposition, their structural and metallurgical behaviour, atomic diffusion, interface structure, their electronic structure, band offsets, electronic defects, charge trapping and conduction mechanisms, reliability, mobility degradation and oxygen scavenging to achieve the thinnest oxide thicknesses. The high $\mathrm{K}$ oxides were implemented in conjunction with a replacement of polcryslliane $\mathrm{Si}$ gate electrodes with metal gates. The strong metallurgical interactions between the gate electrodes and the $\mathrm{HfO}_{2}$ which resulted an unstable gate threshold voltage resulted in the use of the lower temperature 'gate last' process flow, in addition to the standard 'gate first' approach. Work function control by metal gate electrodes and by oxide dipole layers is discussed. The problems associated with high $\mathrm{K}$ oxides on Ge channels are also discussed.
\end{abstract}

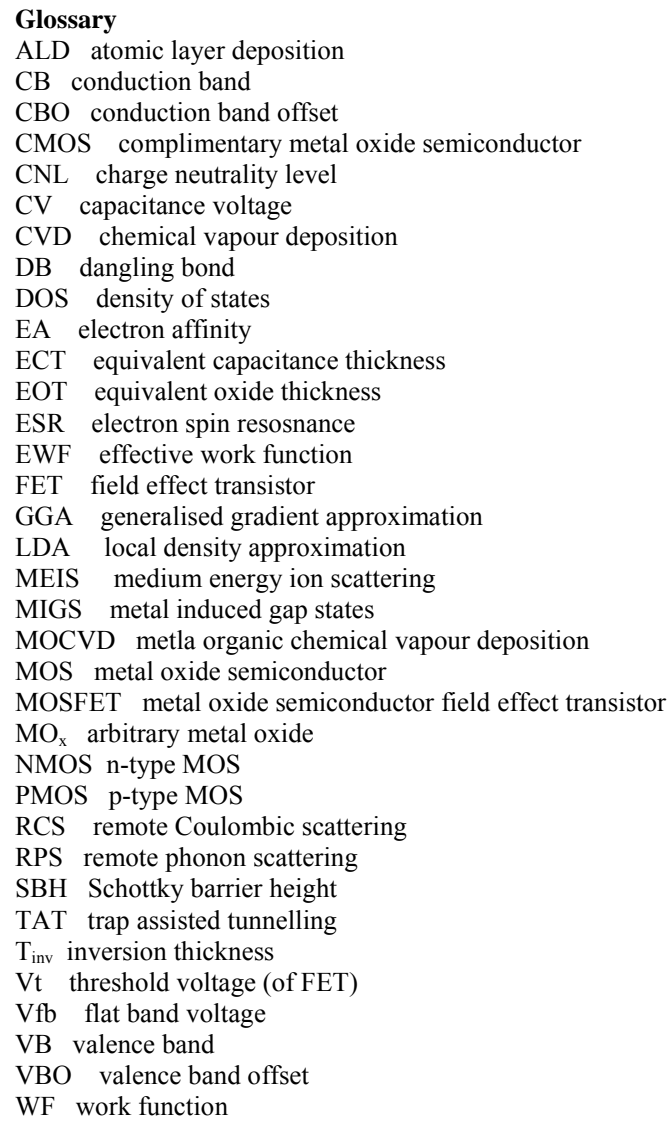


The incorporation of high-K dielectrics with metal gates into a manufacturable, high volume transistor process is the result of tremendous ingenuity and effort by many scientists and engineers [1]. We review that progress in this article, with an emphasis on the key developments in the high-K/metal gate stack process. We also summarize recent results on incorporation of $\mathrm{Ge}$ in the transistor, which appears to provide a viable route to higher performance technology.

It is more than a decade since high-K gate dielectric research for Si-based transistor technology was first reviewed [2,3]. We begin this review with a brief summary of the scaling issues that drove the incorporation of high-K dielectrics and metal gate technologies. We then discuss the materials chemistry of the high-K dielectrics employed in transistor fabrication. This is followed by a review of the current understanding of the high-K/semiconductor interfacial bonding. Defects, which play an important role in high-K dielectrics, are then discussed. Of course, the electrical performance of the transistor is paramount, and we review the recent developments, including work function control for the transistor gate stack.

\subsection{Scaling and gate capacitance}

The complementary metal oxide semiconductor (CMOS) field effect transistor (FET) made from silicon is the most important electronic device. This has arisen because of its low power consumption and its continuing performance improvement over forty years following Moore's Law of scaling. This "law" notes that the number of devices on an integrated circuit increases exponentially, doubling every 2-3 years. The minimum feature size in a transistor decreases exponentially each year (Fig. 1).

Until recently the materials and elements used in CMOS technology were very few - Si, O, P, Al, $\mathrm{B}, \mathrm{H}$ and $\mathrm{N}$. Now the situation has reversed. New materials are being introduced in many areas, from $\mathrm{Cu}$ instead of Al for interconnects, low dielectric constant materials such as $\mathrm{SiOCH}$ for inter-metal dielectrics, silicides as contact metals, and diffusion barrier materials such as TiN.

The $\mathrm{SiO}_{2}$ layer used as the gate dielectric is now so thin $(\sim 1.2 \mathrm{~nm})$ that the gate leakage current due to direct tunnelling of electrons through the $\mathrm{SiO}_{2}$ becomes too high, exceeding $1 \mathrm{~A} / \mathrm{cm}^{2}$ at $1 \mathrm{~V}$ (Fig. 2). This means that the static power dissipation would be unacceptable [2-13]. In addition it becomes increasingly difficult to make such thin films, and they become increasingly unreliable. Thus, $\mathrm{SiO}_{2}$ had to be replaced.

An FET is a capacitance-operated device, where the FET source-drain current depends on the gate capacitance, which is most simply expressed as

$$
\mathrm{C}=\frac{\varepsilon_{0} K A}{t}
$$

where $\varepsilon_{0}$ is the permittivity of free space, $\mathrm{K}$ is the relative dielectric constant, $\mathrm{A}$ is the area and $t$ is the oxide thickness. Tunnelling currents decrease exponentially with increasing distance. Hence, the solution to the tunnelling problem is to replace $\mathrm{SiO}_{2}$ with a physically thicker layer of new material of higher $\mathrm{K}$, as shown in transmission electron microscope images Fig 3. This will keep the same capacitance, but decrease the tunnelling current. These new gate oxides are called 'high K oxides'.

For a device designer, as the precise material does not matter, it is convenient to define an electrical thickness of a new oxide in terms of its equivalent silicon dioxide thickness or 'equivalent oxide thickness' (EOT)

$$
\mathrm{t}_{\mathrm{ox}}=\mathrm{EOT}=(3.9 / \mathrm{K}) \mathrm{t}_{\mathrm{HiK}}
$$


Here, 3.9 is the static dielectric constant of $\mathrm{SiO}_{2}$. The objective is to develop high $\mathrm{K}$ oxides to allow scaling to continue to very low EOT values.

The gate leakage problem has been apparent since the late 1990's [14], but the criteria for choosing the oxide were unclear. In about 2001, the choice of oxide had narrowed to $\mathrm{HfO}_{2}$, but the problems of making $\mathrm{HfO}_{2}$ into a successful electronic material were great. Nevertheless, the increasing importance the low-power electronics in cell-phones, lap-tops, and portable electronics mean that the problem had to be solved. Low standby power CMOS requires a leakage current of below $1.5 \times 10^{-2} \mathrm{~A} / \mathrm{cm}^{2}$ rather than just 1 $\mathrm{A} / \mathrm{cm}^{2}$. There were many difficulties in manufacturing high $\mathrm{K}$ oxides /metal-gate stacks but these were gradually overcome. Intel now manufactures the chips with second generation of high $\mathrm{K} /$ metal $\mathrm{K}$ stacks $[1,15]$ and have now implemented high $\mathrm{K}$ for FINFET structures as well.

The second critical issue was the realisation that metallurgical problems would limit the introduction of the high $\mathrm{K} /$ metal gate stack in an exact replica of the $\mathrm{SiO}_{2}$ gate process (gate first) [16], and that instead a 'replacement gate', 'dummy gate', or 'gate last' process [15] would need to be used, to limit the exposure of high $\mathrm{K} /$ metal gate interface to high temperatures.

In a key early paper, Gusev et al [17] identified four key problems for successful introduction of high K oxides -

(1) be able to continue scaling to lower EOTs,

(2) stop the gate threshold instabilities caused by the high defect densities,

(3) limit the loss of carrier mobility in the Si channel when using high K oxides,

(4) control the gate threshold voltage, which resulted in the need for metal gates.

\subsection{Metal gates and Effective Capacitance Thickness (ECT)}

Viewing the $\mathrm{Si}$ - gate stack band diagram of a MOSFET (Fig. 4), the gate capacitance is the series combination of three terms, the oxide capacitance, the depletion capacitance of the gate electrode, and the capacitance of the Si channel carriers. These three capacitances add as (Fig 5)

$$
1 / \mathrm{C}=1 / \mathrm{C}_{\mathrm{ox}}+1 / \mathrm{C}_{\mathrm{D}}+1 / \mathrm{C}_{\mathrm{Si}}
$$

As $\mathrm{C}$ varies as $1 / \mathrm{t}$, capacitors in series combine as a sum of effective distances. Thus we can define an 'effective capacitance thickness' ECT of the whole gate stack as

$$
\mathrm{ECT}=\mathrm{t}_{\text {inv }}=\mathrm{EOT}+\mathrm{t}_{\text {gate }}+\mathrm{t}_{\mathrm{Si}}
$$

ECT is also known as the inversion thickness $t_{\text {inv }}$.

The channel capacitance $\mathrm{C}_{\mathrm{Si}}$ arises because the 2-dimensional electron gas of carriers in the channel cannot lie infinitely close to its surface, but delocalises a few Angstroms into the Si. This capacitance contribution is intrinsic and cannot easily be changed. High-k dielectric materials are often first evaluated using a MOS capacitor structure, and sunsequently utilizing a transistor structure measuring the substrate (depletion) and source/drain (inversion) capacitance ("Split CV") independently [18,19].

Previously, the gate electrode was made out of degenerately doped polycrystalline silicon (poly-Si). This is stable at high temperatures and compatible with $\mathrm{SiO}_{2}$. Poly-Si is a reasonable metal, but it is not a good enough metal as its relatively low carrier density gives a depletion depth of a few $\AA$. In contrast, a good metal has a much higher carrier density and a depletion depth of only $0.5 \AA$. This depletion effect is removed by replacing poly-Si with a normal metal. The effect on ECT of the replacement of $\mathrm{SiO}_{2}$ by a high $\mathrm{K}$ oxide and poly-Si gate electrode by a metal is shown schematically in Fig. 5. As noted below, the work function of the metal is a critical property to enable proper NMOS or PMOS FET operation.

The ECT is the only thickness that can be measured directly, by capacitance-voltage (CV) methods. On the other hand, EOT can only be estimated by, for example, electron microscopy measurements of oxide thickness, or by back calculating from ECT. EOT is useful, but is not a direct electrical parameter. 
A metal gate material must also be carefully chosen. It is chosen primarily for its work function and its thermal robustness, as discussed in detail in section 6 .

\section{Choice of High K oxide}

Silicon dioxide is the main reason that microelectronics uses $\mathrm{Si}$ technology and not another semiconductor. As a semiconductor, $\mathrm{Si}$ has average performance, but in most respects $\mathrm{SiO}_{2}$ is an excellent insulator. $\mathrm{SiO}_{2}$ has the key advantage that it can be made from $\mathrm{Si}$ simply by thermal oxidation, whereas every other semiconductor ( $\mathrm{Ge}, \mathrm{GaAs}, \mathrm{GaN}, \mathrm{SiC} \ldots$ ) has a poor native oxide or poor interface with its oxide. $\mathrm{SiO}_{2}$ is amorphous, has very few electronic defects and forms an excellent, abrupt interface with $\mathrm{Si}$. (The abruptness of the interface is seen by the absence of intermediate valence states in the $\mathrm{Si}$ photoemission core spectra.) $\mathrm{SiO}_{2}$ can be etched and patterned to a nanometer scale. Its only problem is that it is possible to tunnel across it when very thin. Hence, we must loose these advantages of $\mathrm{SiO}_{2}$ and start to use a new high K oxide. We can in principle choose it from a large part of the Periodic Table.

The requirements of a new oxide are six-fold $[2,5,9]$;

1. Its $\mathrm{K}$ value must be high enough to use economically for a reasonable number of scaling nodes.

2. The oxide is in very close contact to the Si channel, so it must be thermodynamically stable with it.

3. It must act as an insulator, by having band offsets with $\mathrm{Si}$ of over $1 \mathrm{eV}$ to minimise carrier injection into its bands [20]

4. It must be kinetically stable, and able to be processed at $1000^{\circ} \mathrm{C}$ for 5 seconds (in gate first flow).

5. It must form a good electrical interface with Si.

6. It must have few electrically active defects.

Interestingly, once $\mathrm{SiO}_{2}$ is replaced as the dielectric, a key performance advantage of $\mathrm{Si}$ is lost, so other semiconductors such as Ge or III-Vs can now be considered for use as the channel. Silicon continues to provide very useful mechanical properties however, and such properties must be considered in the course of fabrication with automatic-loading tools. Other semiconductors are less abundant and have poor (brittle) mechanical properties. Moreover, as the industry is tooled for large area Si wafers with diameters expected to be at least $450 \mathrm{~mm}$ in the next decade, it will remain a dominant material for integrated circuit manufacturing in all aspects until the end of the technology roadmap.

\section{$2.1 \quad$ K value}

The first key requirement is that the oxide's $\mathrm{K}$ value should be over 12, preferably $25-35$. There is a trade-off between $\mathrm{K}$ value and the band-offset condition, which requires a reasonably large band gap. Table 1 and Fig. 6 shows that an oxide's $\mathrm{K}$ value tends to vary inversely with its band gap, so we must accept a relatively low $\mathrm{K}$ value [20]. There are numerous oxides with very large $\mathrm{K}$, such as $\mathrm{Ta}_{2} \mathrm{O}_{5}$ or $\mathrm{SrTiO}_{3}$, which were candidates for DRAM capacitor dielectrics, but their band gaps are too small.

The static dielectric constant is the sum of the electronic and lattice contributions, $\kappa=\kappa_{\mathrm{e}}+$ $\kappa_{\text {lattice. }}$. The electronic component $\kappa_{\mathrm{e}}$ is also the optical dielectric constant $\varepsilon_{\infty}$ and it equals the square of the refractive index, $n-\kappa_{e}=\varepsilon_{\infty}=n^{2} . \varepsilon_{\infty}$ values are typically in the range 4-5 for the wide gap oxides of interest. Thus they are not the main source of the high $\mathrm{K}$. The large static dielectric constant arises from the lattice contribution,

$$
\kappa-n^{2}=\sum \frac{N e^{2} Z_{T}^{*}}{m \omega_{T O}{ }^{2}}
$$

Here, $\mathrm{N}$ is the number of ions per unit volume, e is the electronic charge, $\mathrm{Z}_{\mathrm{T}} *$ is the ion's transverse effective charge, $\mathrm{m}$ is the reduced ion mass, $\omega_{\mathrm{TO}}$ is the frequency of the transverse optical phonon 
and the sum is over the ions. Large values of $\kappa_{1}$ occur when $Z^{*}$ is large and/or the frequency of a polar optical mode $\omega_{\text {TO }}$ is small. It often arises in materials that are incipient ferroelectrics.

\section{$2.2 \quad$ Thermodynamic Stability}

The second requirement arises from the condition that the oxide must not react with $\mathrm{Si}$ to form either $\mathrm{SiO}_{2}$ or a silicide by the reactions,

$$
\begin{aligned}
& \mathrm{MO}_{2}+\mathrm{Si}=\mathrm{M}+\mathrm{SiO}_{2} \\
& \mathrm{MO}_{2}+2 \mathrm{Si}=\mathrm{MSi}+\mathrm{SiO}_{2}
\end{aligned}
$$

This is because any $\mathrm{SiO}_{2}$ layer increases the EOT and negates the effect of the new oxide, while silicides formed by (7) are metallic and would short circuit the channel.

This condition requires that the oxide have a higher heat of formation (per $\mathrm{O}$ atom) than $\mathrm{SiO}_{2}$ itself as noted by Hubbard and Schlom [21] and Schlom and Haeni [22] found that this restricts choices to very few oxides, from columns II, III and IV of the Periodic Table. These oxides are $\mathrm{SrO}, \mathrm{CaO}, \mathrm{BaO}, \mathrm{Al}_{2} \mathrm{O}_{3}$, $\mathrm{ZrO}_{2}, \mathrm{HfO}_{2}, \mathrm{Y}_{2} \mathrm{O}_{3}, \mathrm{La}_{2} \mathrm{O}_{3}$ and the lanthanides. It excludes some otherwise useful oxides such as $\mathrm{Ta}_{2} \mathrm{O}_{5}$, $\mathrm{TiO}_{2}$ and the titanates $\mathrm{SrTiO}_{3}$ and $\mathrm{BaTiO}_{3}$. The group II oxides $\mathrm{SrO}$ etc are less useful as they have rather low $\mathrm{K}$ values. Hence this leaves us with $\mathrm{Al}_{2} \mathrm{O}_{3}, \mathrm{ZrO}_{2}, \mathrm{HfO}_{2}, \mathrm{Y}_{2} \mathrm{O}_{3}, \mathrm{La}_{2} \mathrm{O}_{3}, \mathrm{Sc}_{2} \mathrm{O}_{3}$ and some lanthanides such as $\mathrm{Pr}_{2} \mathrm{O}_{3}, \mathrm{Gd}_{2} \mathrm{O}_{3}$ and $\mathrm{Lu}_{2} \mathrm{O}_{3}$.

$\mathrm{Zr}$ and $\mathrm{Hf}$ are both from column IV and are generally believed to be two very similar elements. However, it was subsequently found that $\mathrm{ZrO}_{2}$ is slightly more reactive with $\mathrm{Si}[23,24]$ and can form the silicide, $\mathrm{ZrSi}_{2}$. An example is shown in Fig. 7. For this reason, $\mathrm{HfO}_{2}$ was preferred over $\mathrm{ZrO}_{2}$, particulary for the higher thermal budget gate-last case.

Of the other binaries, $\mathrm{La}_{2} \mathrm{O}_{3}$ has a slightly higher $\mathrm{K}$ than $\mathrm{HfO}_{2}$, but is hygroscopic. $\mathrm{Al}_{2} \mathrm{O}_{3}$ has a rather low $\mathrm{K}$ value and high defect density. $\mathrm{Y}_{2} \mathrm{O}_{3}$ and $\mathrm{Lu}_{2} \mathrm{O}_{3}$ have a lower $\mathrm{K}$ than $\mathrm{La}_{2} \mathrm{O}_{3}$. The other lanthanides $\mathrm{Pr}_{2} \mathrm{O}_{3}, \mathrm{Gd}_{2} \mathrm{O}_{3}$ etc are comparable to $\mathrm{La}$ [25-28] but have no particular advantage.

The interface between $\mathrm{HfO}_{2}$ and the $\mathrm{Si}$ substrate has undergone considerable scrutiny in the last decade. Thermodynamically, one anticipates $\mathrm{HfO}_{2}$ to be stable on direct contact with $\mathrm{Si}$, but kinetic effects can alter the interface, potentially resulting in a compositionally graded interface somewhat representative of a silicate [2,29]. The composition of such an interface has spawned some controversy over the years, with some workers indicating that the interface after $\mathrm{HfO}_{2}$ deposition is purely $\mathrm{SiO}_{2}$, [3034] while others indicating the presence of significant amounts of Hf near at the interface [35-38], depending upon the deposition and thermal treatment details.

\subsection{Band offsets}

The high $\mathrm{K}$ oxide must act as an insulator. This requires that the potential barrier at each band must be over $1 \mathrm{eV}$ in order to inhibit conduction by the Schottky emission of electrons or holes into the oxide bands [20,39], as shown in Fig $8 . \mathrm{SiO}_{2}$ has a wide gap of $9 \mathrm{eV}$, so it has large barriers for both electrons and holes. However, for oxides with a narrower band gap like $\mathrm{SrTiO}_{3}(3.3 \mathrm{eV})$, their bands must be aligned almost symmetrically with respect to those of $\mathrm{Si}$ for both barriers to be over $1 \mathrm{eV}$. In practice, the conduction band offset is smaller than the valence band offset. This limits the choice of oxide to those with band gaps over $5 \mathrm{eV}$. The oxides that satisfy this criterion are $\mathrm{Al}_{2} \mathrm{O}_{3}, \mathrm{ZrO}_{2}, \mathrm{HfO}_{2}, \mathrm{Y}_{2} \mathrm{O}_{3} \mathrm{La}_{2} \mathrm{O}_{3}$ and various lanthanides, and their silicates and aluminates [20]. It is interesting that these are the same oxides that pass the thermal stability criterion. This is because a high heat of formation correlates with a wide band gap. On the other hand, the conduction band offset of $\mathrm{SrTiO}_{3}$ is essentially zero $[20,40]$, so this oxide is unsuitable. 
The early leakage current data for various high $\mathrm{K}$ oxides are plotted as a function of EOT in Fig. 8 for typical high $\mathrm{K}$ oxides, $\mathrm{HfO}_{2}$ from [17], for $\mathrm{ZrO}_{2}$ from Gusev et al [41], for $\mathrm{Al}_{2} \mathrm{O}_{3}$ of Gusev [17] and Guha [42,43], for $\mathrm{La}_{2} \mathrm{O}_{3}$ from Iwai [26] and data for $\mathrm{HfO}_{2}$ films with poly-Si electrodes and $\mathrm{HfO}_{2}$ with TiN electrodes, from Tsai [44].

Device scaling will require the use of ever-smaller EOTs. Yeo [45] defined a Figure of Merit, $k$, for direct tunnelling, which combines the barrier height $\phi$, tunnelling mass $\mathrm{m}^{*}$ and dielectric constant $\mathrm{K}$,

$$
\begin{aligned}
& J=J_{0} \exp (-2 k . t) \\
& k=\left(2 m^{*} \phi\right)^{1 / 2}(K / 3.9)
\end{aligned}
$$

where $t$ is the EOT. Lanthanides have the lowest leakage in Fig 9 and the highest figure of merit because they have the largest conduction band (CB) offset. Hf alloys are presently preferred because lanthanides are hygroscopic and because they give low gate threshold voltages for both FET polarities, nFET and pFET.

\section{$2.4 \quad$ Kinetic Stability}

The fourth condition is kinetic stability, that in a gate-first process the oxide must withstand the dopant activation anneal for $5 \operatorname{secs}$ at $1000^{\circ} \mathrm{C}$. We must choose to use a crystalline or amorphous oxide. If an amorphous oxide is desired, this is a strenuous condition as most high $\mathrm{K}$ oxides are not good glass formers, unlike $\mathrm{SiO}_{2}$. In particular, $\mathrm{HfO}_{2}$ and $\mathrm{ZrO}_{2}$ crystallise at lower temperatures (400C) and, would be nano-crystalline in use.

The crystallisation problem can be circumvented by alloying the oxide with a glass former $-\mathrm{SiO}_{2}$ or $\mathrm{Al}_{2} \mathrm{O}_{3}$ - giving either a silicate or an aluminate $[29,46]$. This retains a stability against crystallisation up to nearly $1000^{\circ} \mathrm{C}$. However, silicates have significantly smaller $\mathrm{K}$ values. The addition of nitrogen is beneficial as it further lowers diffusion rates and raises crystallisation temperatures, so that Hf silicates can then pass this criterion [47].

The arguments against poly-crystalline oxides are that its grain boundaries might cause higher leakage currents, act as dopant diffusion paths, and lead to fluctuating $\mathrm{K}$ values from grain to grain. On the other hand, Lee [48] and Kim [49] found that leakage currents of amorphous and nanocrystalline $\mathrm{HfO}_{2}$ are similar, so there was no specific conduction along grain boundaries.

There have been extensive measurements of the atomic diffusion rates of $\mathrm{Hf}, \mathrm{O}, \mathrm{B}$ and $\mathrm{P}$ in $\mathrm{HfO}_{2}$ and Hf silicate by secondary ion mass spectroscopy (SIMS), Medium Energy Ion Scattering (MEIS) and nuclear reaction profiling [50-55]. The addiotn of nitrgoen to $\mathrm{HfO}_{2}$ or $\mathrm{Hf}$ silicate is also very effective in reducing diffusion rates and also increasing crystallisation temperatures. Nitrogen has other benefits on the electrical properties that are covered in section 5.3.

\subsection{Interface Quality and Defects}

The high electrical quality of the $\mathrm{Si}: \mathrm{SiO}_{2}$ interface was the key advantage of $\mathrm{Si}$ as a semiconductor. The interface is essentially atomically abrupt with few sites with intermediate valence; the Si atoms are either in $\mathrm{Si}$ or in $\mathrm{SiO}_{2}$ as seen by XPS core level spectra [56,57]. The interface roughness consists of atomic steps spaced apart by $20 \mathrm{~nm}$ in the highest quality oxide.

The electrical quality of the $\mathrm{Si}$ : high $\mathrm{K}$ interface must be of the highest quality in terms of roughness and absence of defects in order to avoid scattering cariers. Electrically active defects are defined as atomic configurations which give rise to electronic states in the oxide band gap which can trap carriers. Typically, these are sites of excess or deficit of oxygen or impurities. They can be at the interface or in the oxide layer.

Defects are undesirable for four reasons. Firstly, charges trapped in defects shift the gate threshold voltage of the transistor, $\mathrm{V}_{\mathrm{T}}$, the voltage at which it turns on. Secondly, the trapped charge changes with 
time so $\mathrm{V}_{\mathrm{T}}$ shifts with time, leading to instability of operating characteristics. Thirdly, trapped charge scatters carriers in the channel and lowers the carrier mobility. Fourthly, defects cause unreliability; they are the starting point for electrical failure and oxide breakdown.

$\mathrm{SiO}_{2}$ is an almost ideal insulating oxide, having a low concentration of defects which give rise to states in the gap. This is fundamentally because of its low coordination number, so that its bonding can relax and re-bond any broken bonds at possible defect sites. Most of the remaining defects are readily passivated by hydrogen, as seen in electron spin resonance [58-61].

The high $\mathrm{K}$ oxides differ from $\mathrm{SiO}_{2}$ in that they are not intrinsically low defect density materials. Whereas $\mathrm{SiO}_{2}$ has a random network which can relax to heal defect sites, $\mathrm{HfO}_{2}$ and $\mathrm{ZrO}_{2}$ come from a family of fast ion conductors, with potentially large $\mathrm{O}$ vacancy concentrations. Hence, much of presentday engineering consists of pragmatic strategies to reduce oxide defect densities by process control and annealing.

On the other hand, the interface between $\mathrm{Si}$ and the high $\mathrm{K}$ oxide always has an $\mathrm{SiO}_{2}$-rich interfacial layer, it is not an abrupt interface. This allows lower interfacial defect densities to exist in practice. However, after 6 years of further scaling, EOT is reaching values below $0.7 \mathrm{~nm}$, and near-abrupt interfaces are close to being used.

\subsection{Metal gate vs poly-silicon gate}

While the effective capacitance thickness (eqn 4) indicated that scaling would eventually require the replacement of $\mathrm{SiO}_{2}$ by a high $\mathrm{K}$ oxide, and of the poly-Si gate by a metal gate, it was expected that the two technological changes could occur separately. However, it became clear that there was a reaction between $\mathrm{Si}$ and high $\mathrm{K}$ oxide. It was found that the reducing ambient during the CVD deposition poly-Si from silane causes a gross reduction of the $\mathrm{ZrO}_{2}$ or $\mathrm{HfO}_{2}$, leading to silicide formation $[23,62,63]$, as in Fig 7. It was realised that high $\mathrm{K}$ gate oxides and metal gates must be introduced simultaneously.

The second factor was 'Fermi level pinning' which will be described later, and was initially attributed to the formation of $\mathrm{Hf}-\mathrm{Si}$ bonds at the $\mathrm{HfO}_{2}$ /poly-Si gate interface [64-66]. Thus, it was decided to introduce high $\mathrm{K}$ oxies and metal gate simultaneously [1].

\subsection{Gate First/Gate Last Process}

The interface between the gate electrode and the gate oxide is at least as critical to the FET performance as that between channel and the oxide. This interface sets the gate threshold voltage $\mathrm{V}_{t}$, the turn-on voltage of the FET. Whereas the poly-Si gate electrode is refractory and compatible with $\mathrm{SiO}_{2}$, the atoms in $\mathrm{HfO}_{2}$ diffuse much more easily and reactions with metal gates occur at lower temperatures. The details are covered later, but the realisation that such reactions will make the $V_{t}$ very difficult to control led to the decision to develop a 'gate last' process.

The gate first process (Fig 10a) follows the same process flow as with a $\mathrm{SiO}_{2}$ gate oxide [62]. In gate first, we sequentially deposit a gate oxide layer, gate work function tuning layer, and gate metal. The source and drain doping implants are carried out, and the dopant activation anneal to $1000 \mathrm{C}$ is carried out. This annealling temperature is too high for many gate stacks.

In the gate last process, (Fig 10b), the gate oxide is deposited followed by a poly-Si dummy gate $[15,67]$. The source drain dopant implants are carried out, followed by the dopant activation anneal. An interlayer dielectric is deposited for mechanical stability. This is then planarised by chemical mechanical polishing (CMP) down to the level of the top of the dummy gate. The dummy gate is then etched out, and the metal gate layers are then deposited into this hole, followed by an $\mathrm{Al}$ fill. An anneal to $500 \mathrm{C}$ is applied. In this way, the gate metal is not exposed to the $1000 \mathrm{C}$ temperature anneal.

Variant 2 of the gate-last process etches off both the dummy gate and a 'dummy gate oxide', and replaces both with new gate oxide and gate metal. 
The great advantage of $\mathrm{SiO}_{2}$ was that it can be grown by high temnperature thermal oxidation of the $\mathrm{Si}$ substrate. In contrast, high $\mathrm{K}$ oxides must be deposited. Previously, deposited oxides were never as good as $\mathrm{SiO}_{2}$ thermal oxides, but they now have sufficient reliability to be used in transistor technology.

Several deposition methods have been employed in researching high-k dielectrics [68-70]. Sputtering is a physical vapour deposition (PVD) method. Its advantage is that it is broadly available and can produce pure oxides. A disadvantage is that oxides are insulators, so that sputtered oxides will have plasma-induced damage. Also, PVD is line of sight, so it does not give good conformal coverage. A first metal layer can be used to limit the interfacial layer formation and stop over-oxidation. Sputtered films are often used to initially examine dielectric properties prior to further work using CVD or ALD techniques as specialized precurosors are not required.

Another method to produce high purity thin oxides is to deposit metal by electron beam evaporation, which is controllable to small thickness, and then oxidise the metal by ozone or UV assisted oxidation. The advantage is that this produces less damage than oxide sputtering. Ideally, the oxygen partial pressure should be controlled to oxidise just the metal, but not the $\mathrm{Si}[71,72]$.

The preferred methods are chemical vapour deposition (CVD) and atomic layer deposition (ALD). CVD uses a volatile metal compound as a precursor, which is introduced into the chamber and oxidised during deposition onto the substrate. The advantages of CVD are that it is already widely used in the electronics industry for insulator deposition, it gives conformal coverage over complex shapes, and the growth rate is controllable. The conformality property is particularly valuable, as it allows deposition even on complex geometries such as the FinFET.

The most preferred deposition method is ALD. It is a method of cyclic deposition and oxidation [73-76]. It was first developed to produce conformal, pin-hole free insulating films for electroluminescent displays. Fig 11 shows the chemical reactions schematically for the precursor $\mathrm{ZrCl}_{4}$. The surface is exposed to the precursor $\mathrm{ZrCl}_{4}$, which is absorbed as a saturated monolayer. The excess precursor is then purged from the chamber by an Ar or nitrogen pulse. A pulse of oxidant such as $\mathrm{H}_{2} \mathrm{O}$, $\mathrm{H}_{2} \mathrm{O}_{2}$ or ozone is then introduced which fully oxidises the adsorbed precursor layer into $\mathrm{MO}_{2}$ and a volatile by-product such as $\mathrm{HCl}$. The excess oxidant is then purged by another Ar pulse, and the cycle is repeated.

The effective chemical reactions are

$$
\begin{aligned}
& \mathrm{ZrCl}_{4}+2 \mathrm{OH}_{\text {surface }}=\mathrm{ZrCl}_{2} \mathrm{O}_{2}+2 \mathrm{HCl} \\
& \mathrm{ZrCl}_{2} \mathrm{O}_{2}+2 \mathrm{H}_{2} \mathrm{O}=\mathrm{ZrO}_{2}(\mathrm{OH})_{2 \text { surface }}+2 \mathrm{HCl}
\end{aligned}
$$

The existing $\mathrm{ZrO}_{2}$ surface is assumed to be terminated by $\mathrm{OH}$ groups at about $300^{\circ} \mathrm{C}$. The $\mathrm{ZrCl}_{4}$ chemisorbs exothermically onto the $\mathrm{OH}$ sites by the exothermic elimination of $\mathrm{HCl}$ to form $\mathrm{ZrCl}_{2} \mathrm{O}_{2}$. In the second stage, water reacts with $\mathrm{ZrCl}_{2} \mathrm{O}_{2}$ so that $\mathrm{Cl}$ atoms are eliminated via formation of $\mathrm{HCl}$, leaving a layer of $\mathrm{ZrO}_{2}$ on the surface. It is noted that such precursors are $\mathrm{C}$-free, which may improve insulator properties and reliability.

Each cycle of ALD adds a layer of oxide, which is usually much less than an atomic layer thick, despite its name. The precursor absorption saturates at less than one monolayer because of steric hindrance. This is not a significant disadvantage, it just takes more cycles to grow a certain thickness.

The precursor is designed so that both steps of absorption and oxidation are exothermic. The precursor must undergo self-limiting adsorption, be volatile, high purity, non-toxic, have no gas phase reactions, no self-decomposition, and no etching of the existing oxide. The first precursors for $\mathrm{ZrO}_{2}$ and $\mathrm{HfO}_{2}$ were their chlorides. However, these have a low volatility and tended to clog. They are preferred by industry as they avoid carbon impurities, and industry can handle the corrosive exhaust gases. 
A wide range of organic ALD precursors has now been developed to allow high volatility and less corrosive exhausts [73-76]

The main advantage of ALD is that it can grow the thinnest films of any method, and the most conformal films for step coverage or into deep trenches (Fig 12), but the growth rate is very low. Furthermore, a disadvantage of ALD and MOCVD is that they can introduce impurities such as $\mathrm{C}, \mathrm{H}$ or $\mathrm{Cl}$ into the oxides, depending on the precursor. Post-deposition annealing is needed to densify ALD oxides and remove the impurities.

An early problem with ALD was nucleation. The most inert surface of Si is the H-terminated surface formed by HF-last cleaning. It was found that ALD using $\mathrm{HfCl}_{4}$ or many organic precursors did not nucleate properly on HF-last Si surfaces [77,78]. This meant that oxide films even 3 ML thick were not fully 'closed' but islanded. Nucleation occurred more readily on a slightly pre-oxidised Si surface. Thus, ALD was carried out on a 'chemical oxide' $\left(\mathrm{SiO}_{2}\right)$ surface formed by ozone or oxidative cleaning of $\mathrm{Si}$. The nucleation problems on $\mathrm{Si}-\mathrm{H}$ terminated surfaces were removed by using more sophisticated precursors $[79,80]$.

The various types of ALD precursor are listed in Table 2. The precursors are designed to have the desired volatility, reactivity and stability [73-76,81-84]. The molecules are formed by adding organic ligands. The ligands create the volatility. Volatility is aided by using monomer ligands, which do not pack so easily in the solid state. The metal valence is satisfied by polar bonds from $\mathrm{N}$ or $\mathrm{O}$ atoms. These bonds define the stability and reactivity.

The alkoxides and diketonates are commercially available oxygen containing precursors, of reasonable reactivity. The nitrogen-based ligands are becoming more favoured. It turns out that amido compounds have the desired reactivity to give ALD on oxide surfacers and also to nucleate on $\mathrm{Si}-\mathrm{H}$ terminated surfaces. Beta-diketonates are useful for lanthanide oxides. ALD can also produce metal nitrides for electrodes or diffusion barriers [83].

Ho et al [35] followed the progress of the ALD deposition of $\mathrm{HfO}_{2}$ films over a number of ALD cycles by FTIR, see Fig 13. The film was grown from tetrakis ethyl- methyl amino hafnium (TEMAH) or $\mathrm{Hf}\left(\mathrm{N}\left(\mathrm{CH}_{3}\right) \cdot\left(\mathrm{C}_{2} \mathrm{H}_{5}\right)\right)_{4}$ at $100 \mathrm{C}$ with water as oxidant on an $\mathrm{H}$ terminated $\mathrm{Si}$ surface. First, the infra-red spectrum showed that there is an abrupt interface during growth, with Hf-O-Si bonds gradually replacing $\mathrm{Si}-\mathrm{H}$ bonds. The $\mathrm{HfO}_{2}$ growth progress can be seen from the Hf-O modes. The continuing presence of Si$\mathrm{H}$ shows that there is still some island growth even after growing $3 \mathrm{ML}$ of oxide. A separate broadened and red-shifted $\mathrm{Si}-\mathrm{H}$ mode occurs as the $\mathrm{Si}-\mathrm{H}$ group becomes surrounded by $\mathrm{HfO}_{2}$. The reaction of the precursor, not the oxidant, is thus crucial.

The film was then subjected to thermal annealing. The film had O-D modes ( $D=$ deuterium) from the $\mathrm{D}_{2} \mathrm{O}$ oxidant. The post-deposition anneal is used to densify the film and remove contaminants such as organics, water, etc. It is found that at 400-500C, the O-H modes disappear and $\mathrm{Si}-\mathrm{O}$ modes appear. An interfacial $\mathrm{SiO}_{2}$ layer $0.9 \mathrm{~nm}$ thick has grown. It turns out that ALD leaves an oxygen-rich film. When the oxidant is water, the film contains $-\mathrm{OH}$ groups. Annealing should evolve these as $\mathrm{H}_{2} \mathrm{O}$ molecules. However, as the film densifies, $\mathrm{H}_{2} \mathrm{O}$ molecules are too large to escape. It is only possible for $\mathrm{H}$ to diffuse either as $\mathrm{H}^{+}$ions or $\mathrm{H}_{2}$ molecules, desorbing as $\mathrm{H}_{2}$. Further annealing to higher temperatures expels the excess oxygen, and this creates the interfacial $\mathrm{SiO}_{2}$ layer by oxidising the underlying $\mathrm{Si}$. There is no interfacial layer during growth itself.

Recent advances in ALD have been the ability to deposit $\mathrm{LaAlO}_{3}$ using tris(N,N0diisopropylformamidinate)-lanthanum and trimethyl aluminium and the epitaxial growth of oxides on Ge and III-Vs [84].

\subsection{Alloy Crystallisation}

Silicate and aluminate alloys of $\mathrm{Zr}$, Hf and La oxides are often used instead of the pure metal oxides due to their higher resistance to crystallisation $[29,53,85]$. For $\mathrm{Zr}$ silicate, crystallisation directly to the crystalline $\mathrm{ZrSiO}_{4}$ is inhibited by kinetics. Instead, crystallisation occurs by a phase separation into $\mathrm{ZrO}_{2}$ 
and $\mathrm{SiO}_{2}$ phases, followed by a crystallisation of the $\mathrm{ZrO}_{2}$ component [86]. This can be seen for $\mathrm{HfO}_{2}-$ $\mathrm{SiO}_{2}$ alloys in the high-resolution transmission electron microscope images in Fig 14 for two different compositions from Stemmer [87].

The phase diagram of the $\mathrm{ZrO}_{2}-\mathrm{SiO}_{2}$ system is reasonably well known [88], Fig 15(a). That of $\mathrm{HfO}_{2}-\mathrm{SiO}_{2}$ is similar. The key factor is that $\mathrm{ZrO}_{2}$ and $\mathrm{SiO}_{2}$ liquids are immiscible over a range of composition due to the large ionic charge of Zr. This 'miscibility gap' can be extrapolated to lower temperatures to define a solid phase miscibility gap. It also defines a spinodal region in which the alloy will spontaneously phase separate to lower its free energy [88]. The glass transition temperature is also marked in Fig 15(a). Thus, Zr silicates crystallise by two mechanisms. For 20-60mol\% Zr, silicates crystallise by spinodal decomposition followed by crystallisation. This leads to small grain sizes. Silicates with over $60 \% \mathrm{Zr}$ crystallise by the kinetically limited nucleation and growth of crystalline $\mathrm{ZrO}_{2}$. This was confirmed by extensive TEM and x-ray scattering on Hf silicates by Stemmer [89].

The La silicate phase diagram in Fig 15b) $[86,90]$ is somewhat similar to that of $\mathrm{ZrSiO}_{4}$ except that the two-phase region is further towards $\mathrm{SiO}_{2}$. The main difference is that $\mathrm{La}_{2} \mathrm{O}_{3}$ forms a much more stable silicate, whereas $\mathrm{Hf}$ or $\mathrm{Zr}$ silicates prefer to phase separate into the parent oxides.

\subsection{The interfacial layer}

An interfacial layer of $\mathrm{SiO}_{2}$ or silicate usually exists between the $\mathrm{Si}$ channel and the high $\mathrm{K}$ oxide layer. Fig. 16 shows a cross-sectional of an example [91]. There are advantages and disadvantages to this interfacial layer, as long as its presence and thickness can be controlled. The overall EOT of a layer 1 of $\mathrm{SiO}_{2}$ and a layer 2 of high $\mathrm{K}$ oxide is given by the series capacitance formula,

$$
1 / \mathrm{C}=1 / \mathrm{C}_{1}+1 / \mathrm{C}_{2}
$$

which becomes

$$
\mathrm{EOT}=\mathrm{t}_{\mathrm{SiO} 2}+\mathrm{EOT}_{\mathrm{hiK}}
$$

Thus, an extra $\mathrm{SiO}_{2}$ layer is undesirable as it increases the total EOT. In fact, the $\mathrm{K}$ of $\mathrm{SiO}_{2}$ (3.9) is so small that an $\mathrm{SiO}_{2}$ layer will rapidly use up the EOT allocation. It is a severe limitation to scaling of EOT below $1.0 \mathrm{~nm}$.

The $\mathrm{SiO}_{2}$ layer does not arise from the direct reaction of $\mathrm{HfO}_{2}$ with $\mathrm{Si}$. It arises from the diffusion of $\mathrm{O}$ through the $\mathrm{HfO}_{2}$ layer to oxidise the $\mathrm{Si}$ underneath. The $\mathrm{SiO}_{2}$ layer usually grows during the postdeposition annealing stage, not during growth [92]. It can arise for ALD oxide, because the oxide is initially O-rich and it looses oxygen to oxidise the underlying $\mathrm{Si}$ [35]. It can also occur due to oxygen permeation through the top electrode, if the gate material is porous to oxygen.

There was great advantage in retaining the $\mathrm{SiO}_{2}$ intelayer in the early development of high $\mathrm{K}$ gate stacks, because then the $\mathrm{Si}$ interface is still between $\mathrm{Si}$ and $\mathrm{SiO}_{2}$, and it retains the low defect density and relatively high reliability of this interface [88].

The second reason a $\mathrm{SiO}_{2}$ layer exists is intentional, for beneficial reasons. For example, a 'chemical oxide' acts as a nucleation layer for ALD growth of $\mathrm{HfO}_{2}[79,80]$. The $\mathrm{SiO}_{2}$ layer is also introduced to improve the overall electrical quality of the Si-oxide interface, see section 7. The $\mathrm{Si}_{-} \mathrm{SiO}_{2}$ interface is well understood and can be processed to be high quality. In principle, it can be made with a very low defect concentration, by annealing. $\mathrm{A} \mathrm{SiO}_{2}$ layer spaces the $\mathrm{Si}$ channel from the high $\mathrm{K}$ oxide, which can lessen mobility degradation due remote scattering.

\subsection{Metal Inserted Poly-Silicon gates}

A number of metals used as gate metals such as $\mathrm{W}$ contain oxygen or allow $\mathrm{O}$ diffusion. To stop the metal being a source of oxygen, the gate-first process uses a 'metal inserted poly-Si' (MIPS) gate 
electrode, which is a thin layer of the metal underneath the poly-Si capping layer, in which the Si acts as a diffusion barrier to oxygen [10].

\subsection{Oxygen Scavenging}

The continued scaling of EOT below $1.0 \mathrm{~nm}$ and towards $0.5 \mathrm{~nm}$ requires us to either reduce the thickness of the interfacial layer, or increase the $\mathrm{K}$ values. In 2006-2010 there was considerable work towards developing 'higher $\mathrm{K}$ ' oxides than $\mathrm{HfO}_{2}$, as described in section 4.3. However, Ando et al [93] pointed out that the contribution of the $\mathrm{SiO}_{2}$ based interlayer to EOT is so large than higher $\mathrm{K}$ would have little effect. Ando et al [93-97] then tried the idea of increasing the $\mathrm{K}$ of the interlayer, for example by alloying it with lanthanides. However, there are disadvantages to adding La, as it affects the gate threshold voltage.

It was decided to reduce the interfacial $\mathrm{SiO}_{2}$ layer thickness. The $\mathrm{SiO}_{2}$ can be thinned down by an 'oxide scavenging' process, by placing an oxygen scavenging metal layer such as $\mathrm{Ti}$ or $\mathrm{Hf}$ above the $\mathrm{HfO}_{2}$ to 'suck' out the $\mathrm{O}$ through the $\mathrm{HfO}_{2}$ by annealing [98-102]. The scavenger metal should have an oxide heat of formation per $\mathrm{O}$ atom than is greater than that of $\mathrm{SiO}_{2}$ (Fig 11). Ando [95] and Frank et al [96] have carried out extensive work on this process. This is described in more detail in section 7.3.

\section{$4 \quad$ Bonding and electronic structure 4.1 Nature of Bonding in high $\mathrm{K}$ oxides}

The high $\mathrm{K}$ oxides of interest, except for $\mathrm{Al}_{2} \mathrm{O}_{3}$, are closed shell transition metal oxides [103,104]. $\mathrm{Al}_{2} \mathrm{O}_{3}$ is an s,p bonded oxide. Fig 17(a,b) show the density of states (DOS) anmd screened exchange band structure of corundum $\mathrm{Al}_{2} \mathrm{O}_{3}$ the most stable crystalline phase. The top of the valence band lies at $0 \mathrm{eV}$ and the band gap lies from 0 to $8.8 \mathrm{eV}$ [105]. The bonding in $\mathrm{Al}_{2} \mathrm{O}_{3}$ is more ionic than in $\mathrm{SiO}_{2}$, and its atoms have ionic coordinations of 6 for $\mathrm{Al}$ and 4 for O. However, its electronic DOS does resemble that of $\mathrm{SiO}_{2}$. Its valence band consists mainly of $\mathrm{O} p$ states and a conduction band of mainly $\mathrm{Al} s, \mathrm{p}$ states.

The actual phase of $\mathrm{Al}_{2} \mathrm{O}_{3}$ used in gate stacks is amorphous, with lower mass density and a lower coordination of $\sim 4.5$ for $\mathrm{Al}$ and 3 for $\mathrm{O}[106,107]$. This reduces the band gap of the $\mathrm{Al}_{2} \mathrm{O}_{3}$ to about $6.2-6.6$ $\mathrm{eV}$ [108]. It is possible to model the amorphous phase with one of the lower density, lower symmetry phases such as $\theta-\mathrm{Al}_{2} \mathrm{O}_{3}$ whose band structure is shown in Fig 17(c) [109].

The other high $\mathrm{K}$ oxides are closed shell (fully ionised) transition metal oxides. While many transition metal oxides are notable for their mixed valence, here we only deal with fully ionised metal ions. This means the oxides are wide band gap, and do not suffer from the correlation effects.

The most important high $\mathrm{K}$ oxide is $\mathrm{HfO}_{2}$. $\mathrm{HfO}_{2}$, like $\mathrm{ZrO}_{2}$, has three phases, cubic, tetragonal and monoclinic $[110,111]$. The different metal coordinations of these phases are shown in Fig. 18. For large grain sizes, monoclinic is the most stable phase, and cubic the least stable phase. Fig. 19 shows the band structure of cubic $\mathrm{HfO}_{2}$ and the partial density of states (PDOS) [103]. This phase has an indirect band gap with a maximum at $\mathrm{X}$ formed from $\mathrm{O} p$ states. The conduction band minimum is a $\Gamma_{12}$ state of $\mathrm{Hf} 5 \mathrm{~d}$ orbitals. The valence band is $6 \mathrm{eV}$ wide. The conduction band PDOS is split by the crystal field field into a lower band of e states and an upper band of $\mathrm{t}_{2}$ states $5 \mathrm{eV}$ higher.

The electronic structure of the $\mathrm{ZrO}_{2}$ phases is very similar to that of $\mathrm{HfO}_{2}$ except that the crystal splitting of the $\mathrm{Zr} 4 \mathrm{~d}$ states in the conduction band is smaller than in $\mathrm{ZrO}_{2}$ (Fig 20) [103].

$\mathrm{TiO}_{2}$ is another group IV oxide which has various structures including rutile. Its gap is much narrower than that of $\mathrm{HfO}_{2}$ or $\mathrm{ZrO}_{2}$ at about $3.3 \mathrm{eV}$ [112].

Crystalline $\mathrm{La}_{2} \mathrm{O}_{3}$, has a hexagonal structure in which $\mathrm{La}$ is 7 -fold coordinated, with 4 short bonds and 3 longer bonds. The DOS of $\mathrm{La}_{2} \mathrm{O}_{3}$ in Fig. 21 shows that the valence band is strongly localised on the $\mathrm{O} p$ states and the conduction band is on La d states with some La s,p states starting at $8 \mathrm{eV}$ [103]. The band gap is indirect and $6 \mathrm{eV}$. The valence band is now only $3.5 \mathrm{eV}$ wide, narrower than for $\mathrm{ZrO}_{2}$. The ionicity is higher than in $\mathrm{ZrO}_{2}$. 
Of the other group IIIA metal oxides, $\mathrm{Y}_{2} \mathrm{O}_{3}$ has the cubic bixbyite (defect spinel) structure. This has a large 56 atom unit cell in which there are two types of $\mathrm{Y}$ sites, both 7-fold coordinated. This structure occurs because $\mathrm{Y}$ has a smaller ionic radius than La. The band gap of $\mathrm{Y}_{2} \mathrm{O}_{3}$ is direct and is about $6 \mathrm{eV}$. The valence band is again only $3 \mathrm{eV}$ wide. The partial DOS shows the valence band is largely $\mathrm{O} p$ states. The conduction band minimum has mixed $\mathrm{Y}$ d,s character. The other lanthanides adopt either the $\mathrm{La}_{2} \mathrm{O}_{3}$ or bixbyite structure.

In all these transition metal oxides, the conduction band minimum at $\Gamma$ lies at the metal $\mathrm{d}$ energy, while the top of the valence band are non-bonding $\mathrm{O} 2 \mathrm{p}$ states lying at the $\mathrm{O} 2 \mathrm{p}$ energy. Thus the band gap is a pure ionic gap between $\mathrm{O} 2 \mathrm{p}$ and metal $\mathrm{d}$ states. The band gap is proportional to the metal atomic $\mathrm{d}$ orbital energy [1113].

$\mathrm{ZrSiO}_{4}$ is typical of the transition metal silicates. Crystalline $\mathrm{ZrSiO}_{4}$ (zircon) has the body-centred tetragonal structure. Each $\mathrm{Zr}$ atom has eight $\mathrm{O}$ neighbours. Each $\mathrm{Si}$ has four $\mathrm{O}$ neighbours in a tetrahedral arrangement, and each $\mathrm{O}$ has three neighbours, two $\mathrm{Hf}$ and one $\mathrm{Si}$. These coordinations may carry over to the amorphous phases and amorphous alloys. Its partial DOS is shown in Fig 22. The band gap is about $6.5 \mathrm{eV}$ [103]. The valence band is about $7 \mathrm{eV}$ wide [114-116]. The conduction bands form two blocks. The lower conduction band is due to $\mathrm{Zrd}$ states and lies between $6.5 \mathrm{eV}$ and $8 \mathrm{eV}$, and a second conduction band due to Si-O antibonding states lie above $9 \mathrm{eV}$.

The extended $\mathrm{x}$-ray fine structure spectrum [117] suggest that the coordinations in $\mathrm{Zr}$ silicate alloys do not follow the zircon structure, but are locally more like in pure $\mathrm{ZrO}_{2}$ or pure $\mathrm{SiO}_{2}$. This implies local phase separation as noted by Pignedoli [118].

It is an important general rule that the conduction band of $\mathrm{Zr}$ silicates forms two non-mixing $\mathrm{ZrO}_{2}$-like and $\mathrm{SiO}_{2}$-like bands. The states do not mix because the $\mathrm{Si}$ s,p states and metal d states have different local symmetry. Thus, the $\mathrm{CB}$ minimum of the silicates has a $\mathrm{Zrd}$ character as long as $\mathrm{Zr}$ is present, and the band gap increases only slowly, with very strong bowing below the virtual crystal model. Experiments by Kato [119] confirm this.

Another large class of possible gate oxides are the perovskites like $\mathrm{SrTiO}_{3}$. In the $\mathrm{ABO}_{3}$ structure, the smaller transition metal ion occupies the $\mathrm{B}$ site which is octahedrally coordinated by six oxygens. The oxygens are bound to two B ions, while the A ion is surrounded by twelve oxygen ions. The band gap of $\mathrm{SrTiO}_{3}$ is direct and $3.3 \mathrm{eV}$ wide. Due to this small gap, $\mathrm{SrTiO}_{3}$ itself is not useful as a gate oxide.

There has recently been considerable work on other perovskites with larger band gap. $\mathrm{LaAlO}_{3}$ was the first. It is important because it has a large dielectric constant, and a close lattice match to $\mathrm{Si}$. It is unusual in that the transition metal La occupies the A site and Al occupies the octahedral B site. The partial DOS of $\mathrm{LaAlO}_{3}$ is shown in Fig. 23 [120]. The band gap is $5.6 \mathrm{eV}$ from the spectroscopic ellipsometry of Lim [121]. However, the problem with $\mathrm{LaAlO}_{3}$ is that it looses some of its high $\mathrm{K}$ property when it becomes amorphous.

Following this, numerous combinations of rare earths at $\mathrm{A}$ and $\mathrm{B}$ sites were tried, such as $\mathrm{DyScO}_{3}$ and $\mathrm{LaLuO}_{3}$. They all have band gaps of about $5.6 \mathrm{eV}$, and have quite high dielectric constants, usually above 25 [122]. Fig. 24 shows the PDOS of $\mathrm{LaLuO}_{3}$ which includes the filled $\mathrm{Lu} 4 \mathrm{f}$ states in the valence band.

The other type of perovskite is $\mathrm{SrHfO}_{3}$. This retains the simple 2-4 valence of $\mathrm{SrTiO}_{3}$, but is more useful because it has a band gap of $6.0 \mathrm{eV}$ due to the Hf [123].

These various band structures above have been calculated using the local density approximation (LDA) in the generalised gradient approximation (GGA). The LDA under-estimates the band gap of semiconductors and insulators. Many calculations have since used more advanced methods such as hybrid functionals (HSE or screened exchange) [124-127], or by the GW approximation [128,129]. These give calculated band gaps close to the experimental values.

The high $\mathrm{K}$ oxides all have a very large heat of formation, they are very exothermic oxides. This is seen if we plot theoxide's heat of formation against the work function of the parent metal, as in Fig 25 . The oxide heat of formation is equivalent to the Ellingham diagram at $0^{\circ} \mathrm{K}$. The metal work function is related to the Pauling electronegativity of the metal. It is interesting that there is a rough linear correlation of the heat of formation with the work function, until the heat of formation tends to saturate at large work functions.

\subsection{Dielectric constants}


As noted in section 2.1, the high value of dielectric constant arises from the lattice contribution to $\mathrm{K}$, whereas the electronic contribution to $\mathrm{K}$ is relatively minor. Thus high $\mathrm{K}$ oxides are incipent ferroelectrics. Any desire to increase $\mathrm{K}$ must target the lattice contribution.

The dielectric constants have been calculated in the LDA for the various phases of $\mathrm{HfO}_{2}$ and $\mathrm{ZrO}_{2}$ $[130,131]$. This allows us to understand the differences and the anisotropies. Rignanese [131] found that the tetragonal phase has the largest and most anisotropic $\mathrm{K}$, but not by as much as found by Zhao and Vanderbilt [130]. These calculations were extended to lanthanides such as $\mathrm{Lu}_{2} \mathrm{O}_{3}[126]$.

\subsection{Higher $K$ and Phase control}

The continued scaling of MOSFETs contains high $\mathrm{k}$ oxides would appear to require the development of higher K oxides [133,122,145-153]. High K oxides are incipient ferroelectrics. Nevertheless, amorphousness is incompatible with ferroelectricity, as this requires ordered ionic displacements. Thus, huge $\mathrm{K}$ values are unlikely.

On the other hand, Shannon [134] observed that ionic polarisabilities were additive, not just the electronic part which might be expected from bonding arguments, but even the ionic part. Thus the K value varies with the ionic density of the oxides, and the component ions.

Amorphous $\mathrm{HfO}_{2}$ has a lower $\mathrm{K}$ value than cubic $\mathrm{HfO}_{2}$, as has monoclinic $\mathrm{HfO}_{2}$, as both have a lower density than cubic $\mathrm{HfO}_{2}$. On the other hand tetragonal $\mathrm{HfO}_{2}$ has a higher $\mathrm{K}$ value than cubic $\mathrm{HfO}_{2}$, because of its lower frequency polar lattice modes. There has recently been interest in stabilising these higher $\mathrm{K}$ phases.

The first method is to use the ability of $\mathrm{Zr}$ itself to stabilise its tetragonal phase, and when alloyed with $\mathrm{HfO}_{2}$, as seen by [135-137]. The second method is to dope the oxide with a smaller diameter group IV element such as $\mathrm{Si}$ or Ge $[138,139]$. The key point is that group IV dopants stabilise the tetragonal phase over the monoclinic or cubic phase, while the group III elements like Y stabilise the cubic phase, as used in ceramics and jewelry [140-144]. A doping content of 2-7\% is needed.

Many higher K oxides were synthesied by adding rare earths to the perovskites [145-154]. The important aspect of these oxides is that they combine a higher $\mathrm{K}$ value with a large conduction band offset, so their overall figure of merit (eqn 7) is high. $\mathrm{LaLuO}_{3}$ is unusual because it maintains its high $\mathrm{K}$ value even in its amorphous phase $[122,147,149]$, unlike $\mathrm{LaAlO}_{3}$. These metals are from opposite ends of the lanthanide series and have quite different ionic radii, La is large, Lu is smaller. Fig. 21 shows its density of states [147]. Because of the different ionic radii, $\mathrm{LaLuO}_{3}$ remains amorphous up to about $900 \mathrm{C}$. This makes it one of the most interesting higher K oxides [147,148]. However, as noted in section 3.6, ultimately the higher K route to scaling is unsuccessful, reducing the interlayer thickenss by scavenging is recognised to be the most promising method.

This brings us to the last aspect of phase control. In the same way that the tetragonal phase has the highest $\mathrm{K}$ of the $\mathrm{HfO}_{2}$ phases, the hexagonal phase of trivalent oxides like $\mathrm{La}_{2} \mathrm{O}_{3}$ has a much higher $\mathrm{K}$ than its bixbyite (cubic) phase $[155,156]$.

\subsection{Band Offsets}

The band offset between the oxide and Si defines the barrier for injection of electrons or holes into the oxide bands, Fig 26. The conduction band (CB) offset tends to be the smaller of the two. The CB offset is one of the key criteria in the selection of a gate oxide. It must be over $1 \mathrm{eV}$ to give adequately low leakage current [20]. The band offset is measured by methods such as photoemission.

This band alignment at an interface is controlled by any dipole formed by charge transfer across the bonds at the interface. For two non-interacting surfaces, there is no charge transfer, no dipole, and the conduction band line up is given by the difference between the two electron affinities (the energy of the 
conduction band edge below the vacuum level) (Fig 26) [20]. This is called the Schottky limit. If the surfaces interact, charge transfer occurs across the interface, and the resulting interface dipole modifies this offset. The charge transfer acts to align a reference energy level in each surface. In the limit of strong coupling, known as the Bardeen limit, these levels are fully aligned. The band offset is then given by the difference of this reference energy level below the two conduction bands, and is independent of the vacuum levels.

The band line up at an interface is controlled by a dipole formed by charge transfer across the interface bonds [157-159]. The dipole consists of two components, a component intrinsic to the bulk oxide and a component that depends on the interface chemical bonding. The intrinsic component is of interest because the specific bonding at the interface is usually not known.

We first consider the intrinsic band offset. First, the oxide-semiconductor interface is just a variant of the interface between two semiconductors, with the oxie treated as a wide bandgap semiconductor. Second, a semiconductor-semiconductor interfaces is treated as a special case of a semiconductor-metal interface (which is known as a Schottky barrier). A particular model of dipoles at a metal-semiconductor interface is the metal induced gap states (MIGS) model $[160-162,20]$. This model says that in the energy range of the semiconductor band gap, the travelling wave states of a metal decay into the semiconductor as evanescent states called MIGS, Fig 27(a). These states are filled up to some cutoff energy called the charge neutrality level (CNL). In general, the work function of the metal does not equal the CNL energy of the semiconductor, so that when the two systems come into contact, there is a charge transfer between the two which creates an interface dipole. This dipole now tries to align the semiconductor's CNL with the metal Fermi level. The Schottky barrier height, the energy of the semiconductor conduction band above the metal Fermi level, is then given by

$$
\phi_{\mathrm{n}}=\mathrm{S}\left(\Phi_{\mathrm{M}}-\Phi_{\mathrm{S}}\right)+\left(\Phi_{\mathrm{S}}-\chi_{\mathrm{s}}\right)
$$

where $\Phi_{\mathrm{M}}$ is the metal work function, $\Phi_{\mathrm{S}}$ is the charge neutrality level of the semiconductor measured from the vacuum level, and $\chi_{\mathrm{S}}$ is the electron affinity (EA) of the semiconductor. $\mathrm{S}$ is a dimensionless pinning factor given by $S=d \phi_{n} / d \Phi_{M}$ which defines how strongly the MIGS can force this alignment. $S$ is given in the linear approximation by [163]

$$
S=\frac{1}{1+\frac{e^{2} N \delta}{\varepsilon \varepsilon_{0}}}
$$

where $\mathrm{e}$ is the electronic charge, $\varepsilon_{0}$ is the permittivity of free space, $\mathrm{N}$ is the density of the interface states per unit area and $\delta$ is their extent into the semiconductor. Strong alignment or pinning corresponds to $S=0$, while no pinning corresponds to $\mathrm{S}=0$. Large values of $\mathrm{N}$ and $\delta$ give strong pinning.

Monch [160] found empirically that $S$ in (14) varied with the electronic dielectric constant $\varepsilon_{\infty}$ of the semiconductor as

$$
S=\frac{1}{1+0.1\left(\varepsilon_{\infty}-1\right)^{2}}
$$

This is an empirical rule, in addition to the basic MIGS model.

The MIGS model is then extended to describe the band offsets between two semiconductors A and $\mathrm{B}$, or an oxide and a semiconductor. Charge transfer tends to align the CNL of the bulk oxide and the CNL of $\mathrm{Si}$. The CB offset is then [20],

$$
\phi_{\mathrm{n}}=\left(\chi_{\mathrm{a}}-\Phi_{\mathrm{S}, \mathrm{a}}\right)-\left(\chi_{\mathrm{b}}-\Phi_{\mathrm{S}, \mathrm{b}}\right)+\mathrm{S}\left(\Phi_{\mathrm{S}, \mathrm{a}}-\Phi_{\mathrm{S}, \mathrm{b}}\right)
$$


Here, $\chi_{\mathrm{a}}$ is the electron affinity (EA) of the oxide, $\chi_{\mathrm{b}}$ is the electron affinity of the semiconductor, and $\Phi_{\mathrm{S}, \mathrm{a}}$ and $\Phi_{\mathrm{S}, \mathrm{b}}$ are the CNLs of the oxide and semiconductor respectively. All the energies in eqn (15) are measured from the vacuum level, except $\phi_{\mathrm{n}}$ which is measured from the conduction band edge. $\mathrm{S}$ is again the Schottky barrier pinning factor. Note that for two semiconductors, the MIGS only exist where there are band states on one side of the interface, but not where there is a gap on both sides [164], Fig 27(b).

This CNL model is a zeroth-order, but fully parameterised model of band offsets, in which the CNLs are determined by the bulk electronic structure of oxide and Si. The local bonding at the interface does not enter in this model. The CNL acts like the mean electronegativity of the semiconductor [19].

The CNL is an intrinsic property of the bulk semiconductor. It is not a function of the surface chemistry or reconstruction. It is only a function of the bulk band structure [162,20].

The predicted CB offsets in this model $[20,103,165]$ are given in Fig. 28 for the various oxides. Table 3 compares these to the experimental values measured by photoemission, internal photoemission or barrier tunneling [166-186]. Photoemission measures the VB offset, and this is converted into the CB offset by subtracting the oxide and Si band gaps. Internal photoemission measures the energy from the $\mathrm{Si}$ valence band to the oxide's conduction band, or the Si conduction band to the oxide's valence band, depending on the Si doping and of the polarity of the applied voltage.

It is seen that the predicted and experimental offsets generally agree well. Those for $\mathrm{HfO}_{2}$ and $\mathrm{ZrO}_{2}$ from photoemission agree well $[161,184] . \mathrm{SrTiO}_{3}$ indeed has a small CB offset [166]. Data by Hattori [178] for $\mathrm{La}_{2} \mathrm{O}_{3}$ that agrees well with the prediction of $2.3 \mathrm{eV} . \mathrm{La}_{2} \mathrm{O}_{3}$ and $\mathrm{LaAlO}_{3}$ have a particularly large $\mathrm{CB}$ offsets $[178,182]$. The experimental and calculated vales for $\mathrm{Lu}_{2} \mathrm{O}_{3}$ and $\mathrm{Gd}_{2} \mathrm{O}_{3}$ are also in reasonable agreement [180]. There is good agreement for $\mathrm{Al}_{2} \mathrm{O}_{3}$ made by oxidation [167,168]. For $\mathrm{ALD}^{\mathrm{Al}} \mathrm{O}_{3}$ it is important to use the correct phase $[108,170]$.

It is seen that only $\mathrm{Zr}, \mathrm{Hf}, \mathrm{Al}, \mathrm{Y}$, and $\mathrm{La}$ oxides have $\mathrm{CB}$ offsets over $1 \mathrm{eV}$, which is the minimum needed to limit electron injection. The CB offsets decrease in the order of group III, IV, to IV metal oxides. This is because the CNL of the oxide rises in the gap along the sequence group III to V.

The MIGS model predictions have been extended to oxides on Ge and on GaAs [185]. The predictions generally agree fairly well with the experimental values, found by either photoemission or internal photoemission.

\subsection{Bonding at $\mathrm{HfO}_{2}$-Si Interfaces}

The simple MIGS model of oxide interfaces is surprisingly successful. Nevertheless, it is useful to have a detailed bonding model for direct Si-oxide interfaces. Firstly, the band offset can depend on the interface bonding. Secondly, imperfect interfaces will have defects which can create gap states which trap charge. Third, the control of work functions in the case of metal gates requires this understanding of interface chemistry.

It is necessary to use epitaxial models in order to understand the bonding at these interfaces [187196] . The Si: $\mathrm{HfO}_{2}$ system was used as a model, with reasonably a well lattice-matched interface to cubic

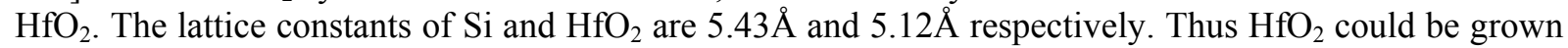
epitaxially on the $\mathrm{Si}(100)$ cube face (as $\mathrm{ZrO}_{2}$ was [192]), with the $\mathrm{HfO}_{2}$ cube face lying directly on top of the $\mathrm{Si}$ cube face. This is expressed as $\mathrm{HfO}_{2}(100) / / \mathrm{Si}(100)$, and with the [001] directions of $\mathrm{Si}$ and oxide parallel, that is $\mathrm{HfO}_{2}[001] / / \mathrm{Si}[001]$.

The ideal (100) surfaces of $\mathrm{HfO}_{2}$ are polar, being oxygen terminated. However, we can construct bulk non-polar units of $\mathrm{HfO}_{2}$ as $\mathrm{O}^{2-} \mathrm{Hf}^{4+} \mathrm{O}^{2-}$ (or 'OHfO') units by assigning O's alternately up or down to give non-polar faces $[189,190]$.

The interface should be insulating, with no gap states, otherwise an FET will not work. We can determine which interface configurations are insulating by an electron counting rule. For the ideal $\operatorname{Si}(100)$ face (Fig 29b), each Si atom would have two dangling bonds (DBs) [189]. These create states in the gap. If we place a non-polar OHfO unit on this (100)Si face, there will be no reaction, and this will still leave Si DB states in the Si gap. These gap states will create a metallic interface. 
If instead we first put an oxygen across the two Si DBs, this forms a Si-O-Si bridge which ties up the $\mathrm{DBs}$ and removes their gap states. We can then add non-polar OHfO units on top. A complete $\mathrm{HfO}_{2}$ lattice can be built up on top of this interface by adding additional non-polar OHfO layers. This interface will have no gap states, and is insulating.

This also works for Hf-termination, Fig 29(c). In this case, the first layer is a HfO unit which is formally $\mathrm{Hf}^{2+} \mathrm{O}^{2-}$ and the $\mathrm{Hf}$ has two unsatisfied valences. These can make two Hf-Si bonds to the Si DBs. This also gives an insulating interface with all valences satisfied. The two examples show that we can obtain insulating interfaces, if polar faces of $\mathrm{HfO}_{2}$ are used. The three cases of O-rich, non-polar $\mathrm{O}$ and Hf terminated interfaces are shown in Fig 29.

Detailed calculations confirm the simplified bonding analysis [190]. Some lower symmetry configurations were also studied. Fig. 30(a) shows the ideal Si:OOHfO interface, with a double oxygen layer at the interface. Here the interfacial oxygens are initially 6-fold coordinated, bonded to two Si's and four Zr's. These interfacial oxygens relax to form the structure shown from two directions in Fig 30(b). Those oxygens lying in the Si-O-Si bridges relax towards the silicon layer. The other two oxygens relax up towards the $\mathrm{HfO}_{2}$ layer. This interface is denoted the $\mathrm{O}_{4}$. Overall, these interfaces have the same number of oxygen atoms at the interface. Another interface denoted $\mathrm{O}_{3}$ interface is a slightly more stable structure. Finally, an interface shown in Fig 30(c) would be formed by a literal ALD process, but this is less dense and less stable.

The non-polar interface of Fig $29 \mathrm{~b}$ would be metallic, in a high symmetry configuration. In a doubled unit cell, the interface reconstructs to break symmetry and create a band gap.

For Hf-terminated interfaces, the simplest has 6-fold coordinated $\mathrm{Hf}_{6}$ sites, as in Fig. 31(a). This structure relaxes so that the interfacial Hf-Si bond becomes longer. This interface is insulating in the full calculation. Fig 31(b) shows another interface in which $\mathrm{Hf}$ is 10 -fold coordinated,. This bonding is similar to that in $\mathrm{ZrSi}_{2}$. This $\mathrm{Hf}_{10}$ interface is the more stable than the $\mathrm{Hf}_{6}$ interface by $0.3 \mathrm{eV}$ per interface $\mathrm{Si}$ [190].

In summary, the $\mathrm{O}_{4}, \mathrm{O}_{3}$ and $\mathrm{Hf}_{6}$ interfaces are insulating. They have no states in the $\mathrm{Si}$ band gap. The $\mathrm{Hf}_{10}$ interface is metallic, despite formally satisfying the electron counting rule, because it is overcoordinated. Thus, only O-terminated interfaces are useful for devices.

The valence band offsets for the various $\mathrm{Si}: \mathrm{HfO}_{2}$ interface structures can be from the partial density of states of $\mathrm{Si}$ and oxide. The conduction band offsets are not given well by the LDA calculations, due to the LDA band gap error. The CB offset is found from the VB offset by adding the experimental band gap. The VB offset for the various O-terminated interfaces of $\mathrm{ZrO}_{2}$ are found to be similar to the VB offset from from the CNL method. In contrast, the $\mathrm{VB}$ offset for Hf-terminated interfaces has a large variation of $0.9 \mathrm{eV}$ between $\mathrm{Hf}_{10}$ and $\mathrm{Hf}_{6}$, being much larger for $\mathrm{Hf}_{6}$. This is due to a large change in the interface dipole. Summarising, the interface-specific dipole is quite small for the O-terminated interfaces, but large for the Hf-terminated interfaces. These results emphasise how much the interface dipole can depend on termination, and go beyond the MIGS model.

Realistic models of the $\mathrm{HfO}_{2}: \mathrm{Si}(100)$ interface, including interfacial $\mathrm{SiO}_{2}$ layers, have been created by Gavartin [197], Broqvist [198], and Alkauskas et al [126]. The presence of the $\mathrm{SiO}_{2}$ does affect the previous band offset values. The Si termination is similar to that expected from ALD reactions.

\section{$5 \quad$ Electronic structure of Defects}

\section{$5.1 \mathrm{HfO}_{2}$}

A problem with high $\mathrm{K}$ oxides [199] is that they can contain a much higher concentration of defects than in $\mathrm{SiO}_{2}$. The two defects in $\mathrm{HfO}_{2}$ with the lowest formation energy are the oxygen vacancy and the oxygen interstitial, fig 32(a,b), according to Foster et al [200,201]. Metal site defects cost more, because of their higher coordinations.

First, consider the oxygen vacancy in $\mathrm{HfO}_{2}$. Recall that the valence band of $\mathrm{HfO}_{2}$ consists mainly of $\mathrm{O} p$ states and the conduction band consists mainly of $\mathrm{Hf} \mathrm{d}$ states. The electronic structure of the oxygen 
vacancy and oxygen interstitial in $\mathrm{HfO}_{2}$ have been calculated by various groups [200-214, 124,127]. Many used LDA methods. The LDA produces good total energies and structures, but LDA underestimates band gaps, by $30 \%$ in the case of $\mathrm{HfO}_{2}$. It also gives the wrong localisation of gap states even when filled. To correct for the band gap error, methods which go beyond LDA such as hybrid functional must be used. Xiong [124] used the screened exchange (SX) method to calculate the defect excitation energies for $\mathrm{HfO}_{2}$ with a 48 atom supercell. The geometry was relaxed in GGA and the energy levels calculated in SX. This was repeated recently for a 96 atom cell. Similar results were later found by Gavartin [211], Broqvist [127], Choi [212] and Lyons [213].

The $\mathrm{O}$ vacancy creates singly degenerate gap state of near spherical $\mathrm{A}_{1}$ symmetry. It is occupied by 2 electrons for the neutral vacancy. The state is strongly localised on the d orbitals of the adjacent $\mathrm{Hf}$ ions. In c- $\mathrm{HfO}_{2}$, the relaxed neutral vacancy gives a gap state at $3.8 \mathrm{eV}$ above the oxide VB, in Fig 30. The ionic positions do not relax much from their ideal positions in the neutral vacancy, the Hf-Hf distance is $3.59 \AA$ compared to $3.54 \AA$ in bulk $\mathrm{HfO}_{2}$.

At the positive vacancy, $\mathrm{V}^{+}$, the $\mathrm{A}_{1}$ defect state is now singly occupied. Its net positive charge causes the adjacent $\mathrm{Hf}^{4+}$ ions to relax outwards from the vacancy. The Hf-Hf spacing becomes $3.74 \AA$. This relaxation causes the defect state to move upwards from $3.2 \mathrm{eV}$ to $4.7 \mathrm{eV}$, Fig 33 . At $\mathrm{V}^{2+}$, the $\mathrm{A}_{1}$ state is now empty. The greater positive charge causes further outward relaxation of the Hf ions so the Hf$\mathrm{Hf}$ separation is $3.90 \AA$ and the gap state rises to $5.2 \mathrm{eV}$.

The vacancy can also trap one or two electrons (despite being an anion vacancy). A trapped electron causes the adjacent $\mathrm{Hf}$ ions to distort asymmetrically (by the Jahn-Teller effect), pulling down an extra, singly-degenerate state of $\mathrm{B}_{1}$ symmetry out of the conduction band. It is singly occupied for $\mathrm{V}^{-}$and doubly occupied for $\mathrm{V}^{2-}$. The $\mathrm{A}_{1}$ state is full in both cases. The complete spectrum of the vacancy levels is summarised in Fig 33.

The formation energy of a defect, $H_{q}$, can be calculated as a function of the Fermi energy $\left(\Delta E_{F}\right)$ from the valence band edge $E_{V}$ and the relative chemical potential $(\Delta \mu)$ of element $\alpha$ [213],

$$
H_{q}\left(E_{F}, \mu\right)=\left[E_{q}-E_{H}\right]+q\left(E_{V}+\Delta E_{F}\right)+\sum_{\alpha} n_{\alpha}\left(\mu_{\alpha}^{0}+\Delta \mu_{\alpha}\right)
$$

where $\left(E_{q}\right)$ is the total energy for the defect cell with charge $q, E_{H}$ is the total energy for the perfect cell, q. $E_{V}$ is the change in energy of the Fermi level when charge $q$ is added and $\mathrm{n}_{\alpha}$ is the number of atoms of species $\alpha$. The oxygen chemical potential $\left(\mu_{\mathrm{O}}\right)$ can vary between the O-rich limit which is taken to be that of the $\mathrm{O}_{2}$ molecule $\mu_{\mathrm{O}}=0$ to the O-poor limit which corresponds to the $\mathrm{Hf}: \mathrm{HfO}_{2}$ equilibrium, which is $\mu(\mathrm{O})=-5.9 \mathrm{eV}$, the experimental heat of formation of $\mathrm{HfO}_{2}$ per $\mathrm{O}$ atom [214].

Fig 34(a) shows the defect formation energy of the vacancy vs Fermi energy for the O-rich limit, and for the O-poor limit. At each value of Fermi energy we look for the lowest energy charge state, the most stable. The neutral state is the most stable for $\mathrm{E}_{\mathrm{F}}$ between $3.2 \mathrm{eV}$ and $5.2 \mathrm{eV}$. Below $3.2 \mathrm{eV}$, the $\mathrm{V}^{2+}$ is most stable. Above $\mathrm{E}_{\mathrm{F}}=5.2 \mathrm{eV}$, the $\mathrm{V}^{-}$and then $\mathrm{V}^{2-}$ become the stable states.

The formation energies for the O-poor limit are simply those for the O-rich limit displaced downwards, Fig 34(b). In the O-poor limit, the cost of an oxygen vacancy is quite small, only $0.8 \mathrm{eV}$ [214]. This is in fact standard. The cost of an $\mathrm{O}$ vacancy of any oxide is close to zero in the O-poor limit. The collorary is that the formation energy of an $\mathrm{O}$ vacancy in the $\mathrm{O}$ rich limit is roughly the bulk heat of formation per $\mathrm{O}$ atom.

The other main defect in $\mathrm{HfO}_{2}$ is the oxygen interstitial [124,201,207,209,214]. It can have various charge states, see Fig 30. The energy levels were calculated using SX [214]. The simplest configuration is the closed shell species $\mathrm{I}^{2-}$, equivalent to an extra $\mathrm{O}^{2-}$ ion. In this state, the interstitial $\mathrm{O}$ is well separated from the other $\mathrm{O}^{2-}$ ions and it adds filled $\mathrm{O} 2 \mathrm{p}$ states to the valence band. At the $\mathrm{O}^{-}$, removing 1 electron leaves a hole at the VB edge. This radical anion moves slightly closer to another $\mathrm{O}^{2-}$ without actually forming a full $\mathrm{O}-\mathrm{O}$ bond, the $\mathrm{O}-\mathrm{O}$ distance is $2.0 \AA$. The neutral interstitial or $\mathrm{O}^{0}$ has two holes in the $\mathrm{O} 2 \mathrm{p}$ levels. The two holes allow this interstitial to form a true $\mathrm{O}-\mathrm{O}$ bond, giving the dumb-bell shaped superoxy anion $\mathrm{O}_{2}{ }^{2-}$. The $\mathrm{O}-\mathrm{O}$ bond length is $1.49 \AA$. This $\mathrm{O}-\mathrm{O}$ bond creates a filled bonding $(\sigma)$ orbital at 
$-6.0 \mathrm{eV}$ just below the main valence band and an empty antibonding $\left(\sigma^{*}\right)$ orbital at $4.1 \mathrm{eV}$ in the upper gap region. It also has filled double degenerate $\mathrm{p} \pi$ and $\pi^{*}$ orbitals at $-3.0 \mathrm{eV}$ in the valence band and at $+0.3 \mathrm{eV}$ just above the VB edge (Fig 33). Fig 34 shows the formation energy of the $\mathrm{O}$ interstitial for Orich and $\mathrm{O}$-poor conditions.

The $\mathrm{I}^{0}$ could trap a further hole to give the $\mathrm{O}^{+}$interstitial, or superoxyl radical. This $\mathrm{O}^{+}$ion forms a shorter $\mathrm{O}-\mathrm{O}$ bond with a $\mathrm{O}^{2-}$ ion of length $1.39 \AA$, giving a dumbbell $\mathrm{O}_{2}^{-}$ion. This gives rise to an empty $\sigma^{*}$ state in the upper gap at $4.5 \mathrm{eV}$, (Fig 32). It also has a filled $\pi$ state and a half-filled $\pi^{*}$ state. This $\mathrm{O}_{2}{ }^{-}$ ion is called the superoxyl radical. The hole resides in one of the $\pi^{*}$ states, breaking their degeneracy. This radical has a characteristic $\mathrm{g}$ factor and has been seen by electron spin resonance in $\mathrm{HfO}_{2}$ thin films [215].

\section{$5.2 \quad \mathrm{HfO}_{2}$ defects: Experimental observations}

The charge trapping characteristics of $\mathrm{HfO}_{2}$ defects have been intensively measured. The trapping characteristics follow the same curves on repeated cycles, indicating that traps get filled and emptied, with no new defects created [216,217], Fig 35.

Kerber [218] interpreted the trapping curves as fast trapping and detrapping of electrons in the oxide. and that the trapping data were consistent with an electron trap level lying just above the Si conduction band edge. Using electron detrapping data over a wider temperature range, Mitard [219] gave levels at 0.8 $\mathrm{eV}$ and $1.5 \mathrm{eV}$ below the $\mathrm{HfO}_{2}$ conduction band edge, or more accurately at $0.7 \mathrm{eV}$ and $1.3 \mathrm{eV}$ above the midgap of the underlying Si. Cartier [220] noted that the levels measured by 'amplitude charge pumping' (ACP) agreed well with the calculated energy levels of the $\mathrm{O}$ vacancy, and that their density correlated with the oxygen deficiency for variously prepared samples. The energy spectra of the traps can also be derived by inelastic tunnelling spectroscopy [221], charge pumping and CV methods [222]. The density of levels found by ACP correlated linearly with bias stress voltage change measured on differently prepared gate oxides. This indicates that the $\mathrm{O}$ vacancy is the primary cause of the charge trapping instability in $\mathrm{HfO}_{2}$.

Optically, Takeuchi [223] identified an absorption band at $4.5 \mathrm{eV}$ in spectroscopic ellipsometry on $\mathrm{HfO}_{2}$ films oxidised to different degrees. They attribute this to transitions from the valence band to an oxygen vacancy level at $4.5 \mathrm{eV}$. The peak was seen in optical absorption by Nyugen [224]. The transition was seen by cathodo-luminescence in $\mathrm{HfO}_{2}$ films by Walsh [225]. There was concern that the absorption band might correspond to the E2 absorption of the underlying Si substrate, but the other spectroscopic tools suggest that the identification with the vacancy still holds.

The oxygen vacancy is the main mechanism for oxygen diffusion in $\mathrm{HfO}_{2}$ and related oxides, so there have been a number of efforts to correlate vacancy concentrations as a function of oxygen partial pressure by Guha [226,227], McIntyre [228], and of oxygen diffusion measurements by Zafar [229].

The chemical nature of the traps can usually be determined directly by electron spin resonance (ESR). So far, many of the defects seen by ESR in $\mathrm{HfO}_{2}$ on $\mathrm{Si}$ have been related to the interfacial Si dangling bond, the so-called the $\mathrm{P}_{\mathrm{b}}$ centre [230,231]. Kang [215] identified two paramagnetic defects in bulk $\mathrm{HfO}_{2}$ produced by $\mathrm{ALD}$; the $\mathrm{Hf}^{3+}$ ion (an electron trapped at $\mathrm{Hf}^{4+}$ or $\mathrm{V}^{+}$) and the superoxy radical. The problem with the ESR data for the $\mathrm{O}$ vacancy is that the observed ESR $\mathrm{g}$ matrix has an axial symmetry [232] which does not fit the expected spherical symmetry of an $A_{1}$ state. However, it is now realised that LDA gives defect wavefunctions that are too delocalised. Fig. 36(a) shows the symmetric wavefunction found by LDA. If hybrid functionals such as B3LYP or SX are used instead, these methods find a more localised wavefunction and lower symmetry defect, as seen in Fig 36(b), and this is now consistent with the experimental ESR signature [233,208].

The conduction processes in insulating thin films can be classified as limited by bulk or interface prcocesses. Bulk processes include hopping through traps (Poole-Frenkel) and space-charge limited current (SCLC). For $\mathrm{HfO}_{2}$ and $\mathrm{ZrO}_{2}$, Poole-Frenkel hopping may be dominant for thicker films, over 4 $\mathrm{nm}$. For thinner films, Houssa [234] attributes conduction to trap-assisted tunnelling (TAT). Tunnelling 
should be essentially independent of temperature, hopping T-dependent. The technologically relevant films of $\mathrm{HfO}_{2}$ tend to be only $2-3 \mathrm{~nm}$ thick. In this range, TAT occurs for $\mathrm{V}<0.5 \mathrm{~V}[48,235]$, and direct tunnelling occurs for higher voltages. In all cases, conduction is by electrons, because the CB barrier is lower. The relevant traps are the oxygen vacancy levels.

\subsection{Defect Passivation}

The defect densities in $\mathrm{HfO}_{2}$ can be reduced by passivation. The standard method to passivate dangling bond type defects in covalent semiconductors or $\mathrm{SiO}_{2}$ is a forming gas anneal in hydrogen. The hydrogen ties off the dangling bond, and moves the states out of the gap. $\mathrm{HfO}_{2}$ is different, it has ionic bonds and the defects are not dangling bonds. Different strategies are now required.

Annealing in ammonia or nitrogen was found to lower leakage currents, which might be realted to lowering the defect density. Umezawa [236] noted a possible mechanism of nitrogen passivation of $\mathrm{O}$ vacancies in which two nitrogens substituted for oxygen next to a neutral oxygen vacancy, $\mathrm{VN}_{2}$, removed the vacancy-related gap state. The passivation occurs because each substitutional $\mathrm{N}$ would leave a hole in the VB (Fig 37b), so the two electrons of $\mathrm{V}^{0}$ fall into the hole states, making an effective doubly postively charged vacancy [202]. The $\mathrm{Hf}$ ions adjacent to the vacancy relax outwards, as in the simple $\mathrm{V}^{2+}$, but here the relaxation repels the $A_{1}$ level completely out of the gap, Fig 37(a). The configuration now has a 'closed shell', so it should not give rise to gap states, Fig 37(c).

Gavartin [237] studied other configurations of $\mathrm{N}$ in $\mathrm{HfO}_{2}$. These configurations still give gap states, so they are not passivating the defects. Nitrogen introduces N 2p states in the lower gap, which lie above the normal valence band edge [238], so this reduces the local band gap. However, the valence band offset is large, so this does not have a deleterious effect.

The vacancy can also be passivated by the substitution of two Hf sites by a trivalent metal such as La $[239,240]$ (Fig 38). In this case, two La ions are substituting for two Hf ions, next to the $\mathrm{O}$ vacancy. The two trivalent La sites lead to two holes in the valence band, into which the two electrons of the neutral vacancy fall. As above for $\mathrm{N}$, this leaves a closed shell and a $\mathrm{V}^{2+}$ vacancy. As in the case of nitrogen above, the relaxed $\mathrm{V}^{2+}$ pushes its $\mathrm{A}_{1}$ state up out of the gap, into the conduction band. Both these methods can be used to improve the reliability of $\mathrm{HfO}_{2}$.

Fluorine passivation has also been found to be beneficial by Tseng [241] and McIntyre [242], probably because $\mathrm{F}$ might insert into oxygen vacancies. According to calculations, the $\mathrm{F}$ lies in the vacancy, and repels any vacancy states into the oxide conduction band, thereby passivating the vacancy [243] (Fig 34). However, a disadvantage is that too much fluorine will lower K.

It turns out that many inpurirites and defects can be passivated in $\mathrm{HfO}_{2}$ because of the nature of wide band gap systems [244].

\subsection{Defects in $\mathrm{Al}_{2} \mathrm{O}_{3}$}

$\mathrm{Al}_{2} \mathrm{O}_{3}$ is the other widely used high $\mathrm{K}$ oxide. This is partly because of its ability to remain amorphous, and partly because it is a good atomic diffusion barrier. It is widely known to have a fairly high concentration of defect states. This can be advantageous for some memory applications.

The lower symmetry $\theta-\mathrm{Al}_{2} \mathrm{O}_{3}$ phase is used as a model for the amorphous phase [109,245]. Fig 39 shows the defect formation energy as a function of Fermi energy for the $\mathrm{O}$ vacancy. It is seen that the $\mathrm{O}$ vacancy has 5 stable charge states in the gap, $+2,+1,0,-1$, and -2 . This means that there are four transition levels, $+2 /+1,+1 / 0,0 /-1$ and $-1 /-2$. This may be why the defect levels of $\mathrm{Al}_{2} \mathrm{O}_{3}$ appear to be broadly distributed across the gap.

The calculated transition states have ben compared to experimental data. Liu [109] showed that the $0 /-1$ level corresponds to the main hopping level observed in transport experiments [246-249]. Choi [245] has studied the defects in $\alpha-\mathrm{Al}_{2} \mathrm{O}_{3}$.

Defect levels have also been calculated for $\mathrm{La}_{2} \mathrm{O}_{3}$ and the perovskites $\mathrm{LaAlO}_{3}$ and $\mathrm{LaLuO}_{3}$ [250] as shown in Fig. 40. 


\section{$6 \quad$ Metal gates \\ 6.1 Work function requirements of metal gates}

The purpose of the gate electrode in CMOS is to shift the surface Fermi level $\mathrm{E}_{\mathrm{F}}$ of the channel to the other band edge, to invert the transistor. An NMOS FET consists of a p-doped Si channel. A gate electrode of low work function $(\sim 4.05 \mathrm{eV})$ will move its surface Fermi level from the Si valence band to the conduction band, inverting the channel. Similarly, a PMOS device has a n-doped Si channel, and a gate with a work function of $5.15 \mathrm{eV}$ shifts its $\mathrm{E}_{\mathrm{F}}$ to its valence band, inverting the channel. This requires a work function change of $1.1 \mathrm{eV}$, the Si band gap. The work functions of metals are tabulated by Michaelson [251].

For previous MOSFETs with $\mathrm{SiO}_{2}$ dielectric, the gate electrodes were polycrystalline Si highly doped n-type or p-type respectively, for NMOS and PMOS respectively. This gives work functions of 4.05 and $5.15 \mathrm{eV}$, respectively. Poly-Si has the advantage that it is a refractory material, easily deposited, and compatible with $\mathrm{SiO}_{2}$ and the associated process flows. However, doped poly-Si has a limited carrier density, and so it adds a depletion length of $\sim 3 \AA$ to the ECT, as in Fig 4. Real metals have a depletion lengths under $0.5 \AA$, so using them lowers ECT by $3-4 \AA$.

\subsection{Effective Work Functions}

The requirement for the two metal gate replaced doped poly-Si is that they should be able to swing $\mathrm{E}_{\mathrm{F}}$ over that $1.1 \mathrm{eV}$ range of work functions. The key point is that $\mathrm{SiO}_{2}$ is an essentially perfect insulator so that a work function applied on the top of a thin $\mathrm{SiO}_{2}$ layer gives a work function of the same value at the $\mathrm{Si}: \mathrm{SiO}_{2}$ interface. This is not necessarily the case for metals on thin $\mathrm{HfO}_{2}$, for various reasons. The ability to swing the surface work function of the $\mathrm{Si}$ is measured by the $\mathrm{CV}$ plot.

We first recall the description of Schottky barriers and MIGS in section 4.4. The Schottky barrier pinning factor S given by

$$
\mathrm{S}=\mathrm{d} \phi_{\mathrm{FB}} / \mathrm{d} \Phi_{\mathrm{M}}
$$

defines how strongly the Schottky barrier height (SBH) is pinned. If the density of MIGS is large, there is strong pinning and $\mathrm{S}=0$. If the MIGS density is small, there is little pinning and $\mathrm{S}=1$. $\mathrm{SiO}_{2}$ behaves almost like a vacuum, its MIGS are very weak, and there is no pinning, so $\mathrm{S}=1$. This means that metal work function act as if at a vacuum interface.

This is not the case for the $\mathrm{HfO}_{2}$ surface. Using formula (15) gives $\mathrm{S} \sim 0.5$ for $\mathrm{HfO}_{2}$. Some of the experimental data suggests that this value is correct. Therefore, moderate Fermi level pinning was expected for $\mathrm{HfO}_{2}$ on Si. Yeo [252] noted that this would be a disaster for $\mathrm{HfO}_{2}$. Rather than chosing metals with a work function difference of $1.1 \mathrm{eV}$ between nFeT and pFET, we would need a work function swing of $2.2 \mathrm{eV}$ to obtain a SBH swing of $1.1 \mathrm{eV}$. The work function of $\mathrm{Pt}$ is $5.65 \mathrm{eV}$, so that a work function of $3.4 \mathrm{eV}$ would be needed for an nFET gate. This would be an extremely electropositive, reactive metal like Sc.

This led to the use of 'effective work function' (EWF) as a way of defining how much voltage shift a given gate electrode would provide, when referenced to the vacuum level. Formally, it references the surface potential on Si to say the Si conduction band energy, and then adds the Si electron affinity (Fig 42). The EWF or $\Phi_{\mathrm{M}, \text { eff }}$ can also be thought of as linearising the pinning effect, as

$$
\Phi_{\mathrm{M}, \mathrm{eff}}=\Phi_{\mathrm{CNL}}+\mathrm{S}\left(\Phi_{\mathrm{M}, \mathrm{vac}}-\Phi_{\mathrm{CNL}}\right)
$$

where a reference energy such as the $\mathrm{CNL}$ energy $\Phi_{\mathrm{CNL}}$ is used for convenience. 


\subsection{Practical control of the Work Function}

Figs 43 shows some data for the barrier heights of some metals on $\mathrm{HfO}_{2}$ as measured by $\mathrm{CV}$ or by internal photoemission. Some data such as from Afanasev [169,170] suggest that $\mathbf{S} \sim 0.7$. On the other hand, data by Koyama [253] taken with more annealing suggested larger S values. Zafar [254] derived barrier height of metals from a conductivity method and found that the SBHs of three refractory metals (TaSiN, $\mathrm{W}$ and $\mathrm{Re}$ ) on $\mathrm{HfO}_{2}$ corresponded to $\mathrm{S}=1$.

The first extensive compilation of EWFs by Schaeffer [255] in Fig 44 found a low value of S, suggesting a problem. These results suggested some intrinsic Fermi level pinning. Similarly, a Fermi level pinning effect at poly-Si gate / $\mathrm{HfO}_{2}$ interfaces seen by Hobbs $[62,64]$ was attributed to the formation of $\mathrm{Hf}-\mathrm{Si}$ bonds at the $\mathrm{HfO}_{2}$-Si interface [66].

On the other hand, Schaeffer [256] showed that the effective work function of Pt depended reversibly on its treatment by a forming gas (reducing) or oxidising anneals, implying a large extrinsic effect. Cartier [257] showed that the work function of a Re electrode depended greatly on its annealing in reducing or oxidising conditions, as in Fig 45. These data indicate that the WF shifts on p-type metals are an extrinsic effect due to defects or chemistry, not due to MIGS.

Majhi [258] and Wen [259] carried out a detailed study of more refractory metal electrodes (typically TiSiN, TaSiN etc). Because the flat band voltage can depend in general on both charge defects in the oxide and the interfacial potentials, the EWF was extracted usig a more reliable method using terraced oxide or 'wedding cake' samples [260], as in the inset to Fig 46(a). Here, the thickness of the interfacial $\mathrm{SiO}_{2}$ varies, the $\mathrm{HfO}_{2}$ thickness is constant. The data in Fig 46(a) showed that the measured range of EWFs of metals on $\mathrm{HfO}_{2}$ was less than on $\mathrm{SiO}_{2}$, but not hugely less, and more importantly, they could span the band gap of $\mathrm{Si}$, and invert both NMOS and PMOS. This data is replotted in Fig 46(b). By assuming $\mathrm{S}=1$ for metals on $\mathrm{SiO}_{2}$, we extract a value of $\mathrm{S} \sim 0.82$ for metals on $\mathrm{HfO}_{2}$. This then led to an era of searching for manufacturable metal combinations to use as NMOS and PMOS metal gates.

There were two approaches. First, Tseng [261] and Schaeffer [262] found that sufficiently refractory metal carbides such as $\mathrm{TaC}_{\mathrm{x}}$ had a low and stable EWF on $\mathrm{HfO}_{2}$ of about $4.2 \mathrm{eV}$, reasonably useful for nFETs. This method works because $\mathrm{TaC}$ is so strongly bound that it does not react significantly with the $\mathrm{HfO}_{2}$. On the other hand, there was no clear pFET solution using electronegative metals.

The extrinsic pinning effect led to a new industrial solution, gate last vs gate first (Fig 6). The gate first process follows the standard process flow, but with a metal gate replacing the poly-Si gate. This appoach was followed by IBM [263,264]. It is more difficult metallurgically because the gate metal must withstand the full process $1000 \mathrm{C}$ temperature.

Alternatiively, in order to avoid electrode reactions occurring at high temperatures, the gate last process was developed. It makes a dummy poly-Si gate as in the standard process. The $1000 \mathrm{C}$ postdeposition anneal on the channel dopants is carried out, then the dummy gate is removed and the replacement metal gate is put into the gate space. It is then subjected to a much lower temperature anneal of order 550C. This process has more process steps but avoids metallurgical reactions. It requires a high degree of control of the chemical mechanical polishing (CMP) that planarises the dummy gate area to enable its removal.

\subsection{Work function control}

These experimental observations show the need to improve our understanding of Schottky barriers. To understand metal- $\mathrm{HfO}_{2}$ interfaces in more detail, the $\mathrm{SBHs}$ for epitaxial interfaces of various metals on cubic $\mathrm{HfO}_{2}$ were calculated $[265,266]$. This calculation requires that the metal is lattice-matched to the $\mathrm{HfO}_{2}$. Only $\mathrm{Ni}$ and Co are closely lattice-matched to $\mathrm{HfO}_{2}$. For other metals, we force the lattices to match in the interface plane, and allow them to relax normal to the interface. In addition, we take all the metals to be FCC, even if this is not their most stable phase. The vertical dimension and all atomic positions were allowed to relax. 
The barrier heights were calculated from the offset of the valence band local density of states on metal and oxide atoms far from the interface. The offsets can be adjusted for the GGA band gap error by lowering the oxide valence band edge by the $\mathrm{GW}$ correction of $1.23 \mathrm{eV}$ if desired [187].

Interfaces can be polar or non-polar [267,268]. The simplest non-polar (type 1) face of $\mathrm{HfO}_{2}$ is $(110)$, while (111) is a type 2 non-polar face. The (100) face is polar, and can be O-terminated or Hf-terminated. A non-polar (100) face can be made by removing half of the terminating oxygens in the O-rich interface. Fig 47 shows these various interfaces for $\mathrm{HfO}_{2}$ on $\mathrm{Ni}$.

The lattice constant of $\mathrm{HfO}_{2}$ is $\sim 1.4$ times greater than that of Ni. The lattice-matching occurs by rotating the Ni lattice by $45^{\circ}$ on (100). This rotation will also allow matching on (110), but not (111). A modified interface was constructed for (111).

Fig 48 plots the calculated barrier heights of metals on $\mathrm{HfO}_{2}$ against their experimental work function from Michaelson [251] for both the non-polar and polar interfaces. We see that there is a large offset between the barrier height for the two cases; the O-rich interface has a much lower valence band offset, or a higher EWF. Secondly, the slope of band offset versus work function, S, is calculated to be 0.92 , or close to 1 . Thus, first principles calculations find the interface to be essentially unpinned. The fact that the pure metal: $\mathrm{HfO}_{2}$ interface is unpinned is important. It is consistent with the experimental observations of metals on $\mathrm{HfO}_{2}$ as seen in Fig 46 [259].

\subsection{Extrinsic pinning models}

It is clear from the above result and also the ability to vary the gate threshold voltages reversibly by annealing in a hydrogen atmosphere that the pinning is extrinsic. The Fermi level pinning mechanism for electronegative (pFET) metals involves oxygen vacancies and band-bending in the oxide [260-262]. The vacancies are created by the high temperature anneal. The $\mathrm{O}$ vacancy is neutral, and it has an energy level in the oxide near the Si conduction band energy occupied by two electrons [120]. These electrons can gain energy by falling down to the metal $\mathrm{E}_{\mathrm{F}}[269,270]$, which is large for electronegative metals, Fig 49(a). This gives the vacancy levels a positive charge, which causes upward band bending of the oxide layer, thereby reducing the EWF of the gate electrode, referred to the channel, Fig 49(b). However, it turns out that the vacancy formation energy is too large at an $\mathrm{O}$ chemical potential of $\mu_{\mathrm{O}}=0 \mathrm{eV}$, for there to be enough charged $\mathrm{O}$ vacancies to cuase much band bending [269-271]. However, the $\mathrm{HfO}_{2}$ can react with the underlying $\mathrm{Si}$, the $\mu_{\mathrm{O}}$ is greatly reduced, and many vacancies can form.

The simplest way to understand it follows from the formation energy diagram, Fig 50. The formation energy of the vacancy depends on the charge state, via the Fermi energy, but also on the $\mathrm{O}$ chemical potential $\mu_{\mathrm{O}}$. For $\mu_{\mathrm{O}}=0 \mathrm{eV}$, that of the $\mathrm{O}_{2}$ molecule, then the vacancy formation energy is large, $6.8 \mathrm{eV}$. This $\mu_{\mathrm{O}}$ is appropriate for the as-deposited film. However, the $\mathrm{HfO}_{2}$ gate oxide is not as-deposited, it is annealed sitting next to a large excess of $\mathrm{Si}$. This excess can greatly reduce the oxygen chemical potential, once the temperature rises to allow $\mathrm{O}$ diffusion to react with the $\mathrm{Si}$ and form more $\mathrm{SiO}_{2}, \mathrm{Fig} 51$. The O vacancy now is much easier to form [271].

Fig. 50 plots the $\mathrm{O}$ vacancy formation energy for $\mu_{\mathrm{O}}=-4.7 \mathrm{eV}$, the $\mathrm{O}$ chemical potential of the $\mathrm{Si} / \mathrm{SiO}_{2}$ equilibrium. The neutral oxygen vacancy now costs $1.3 \mathrm{eV}$. The $\mathrm{O}$ vacancy formation energy then becomes negative when $\mathrm{E}_{\mathrm{F}}$ lies less than $3.5 \mathrm{eV}$ above the $\mathrm{HfO}_{2}$ valence band, that is for effective WFs larger than $5 \mathrm{eV}$. This leads to the creation of +2 vacancies, which cause band-bending next to the gate electrode. This reduces the EWF of high WF metals. For this reason, obtaining a sufficiently p-type gate electrode is extremely difficult once $\mathrm{HfO}_{2}$ is annealed above the $\mathrm{O}$ diffusion temperature of $\sim 550 \mathrm{C}$.

This model accounts for the observations of Cartier [257] and Schaeffer [256]. The pinning occurs above $\sim 600^{\circ} \mathrm{C}$ because the $\mathrm{O}$ must diffuse to react with the Si. Cartier noted that the pinning can be reversed by $\mathrm{O}$ saturation if the vacancies are back-filled by oxygen [272].

The extrinsic Fermi pinning is most noticeable for p-metals on smaller EOT stacks. This is known as the $V_{t}$ roll-off effect [12], as was shown in Fig 52. Song [273], Akiyama [274], Schaeffer [275] and Bersuker [276] have produced models to explain this effect, based on oxygen transfer. Bersuker [276] attributes rol-off to the formation of positively charged $\mathrm{O}$ vacancies close to the channel. Roll-off limits 
the scalability of pFETs in the gate-first approach, and was a considerable influence in the move to gatelast.

\subsection{Oxide Dipole layers}

When the gate electrode was doped poly-Si, gate voltage control was arranged by changing the EWF of the electrode, by doping it $\mathrm{n}$ - or $\mathrm{p}$-type. The first choice for metal gates was to choose two metals with WFs similar to those of n-type Si and p-type Si. This can lead to difficult lithography.

An alternative option is to have a single, stable metal electode like TiN with a midgap work function, and using an oxide 'capping layer' under the gate to control the net EWF through the gate stack, Fig 53.

It is found that oxides of electropositive group IIA and IIIA metals on top of the $\mathrm{HfO}_{2}$ layer would shift the overall $\mathrm{V}_{\mathrm{FB}}$ of the gate stack towards an n-type value, as achieved by Narayaanan [277], Guha [278], and AlShareef [278-279]. On the other hand, layers of $\mathrm{Al}_{2} \mathrm{O}_{3}$ can be used to shift the $\mathrm{V}_{\mathrm{FB}}$ in a ptype direction $[280,281]$. The shift is roughly proportional to the capping layer thickness. In other words, it does not give a fixed $\mathrm{V}_{\mathrm{FB}}$ value like for example doped poly-Si.

Finding the underlying mechanism of the capping layers was not simple. First, the $\mathrm{LaHfO}_{\mathrm{x}}$ alloy data of Wang [282] showed that the interfacial fixed charge was small, so that the effect was truly a dipole shift. Second, medium energy ion scattering showed that the capping layer did not remain at the upper surface. Under a $1000 \mathrm{C}$ anneal, the La oxide distributes across the dielectric layer (Guha [277]) (Fig 54).

Yamamoto [283] and Iwamoto [284] then showed by a series of alternate depositions, that the interface determining the $\mathrm{V}_{\mathrm{FB}}$ shift is not the top gate-oxide interface, but rather the lower $\mathrm{SiO}_{2}$-high $\mathrm{K}$ interface, because the capping layer oxide had diffused through the $\mathrm{HfO}_{2}$ Fig 55. It was no longer a 'capping layer'. This applies to both $\mathrm{La}_{2} \mathrm{O}_{3}$ and to $\mathrm{Al}_{2} \mathrm{O}_{3}$ dipole layer systems. The effect might be due to the strong reaction of $\mathrm{La}_{2} \mathrm{O}_{3}$ and $\mathrm{SiO}_{2}$ to form a silicate.

Presently three atomic scale mechanisms have been proposed. Guha [267] suggested that the group II or III metals substituting into the $\mathrm{HfO}_{2}$ lattice would create oxygen vacancies, due to their different valence, and the charge oxygen vacancies would cause the dipole shift. Second, Kirsch [285] measured the $\mathrm{V}_{\mathrm{FB}}$ shifts for a range of capping oxides, and found that the EWF shifts correlated with the dipole moment of the M-O bond, expressed on a Pauling scale of ionicities. Third, Jagannathan [286] noted that the $\mathrm{V}_{\mathrm{FB}}$ shifts correlate with the metal electronegativity of the capping oxide, using the group electronegativity concept of Schaffer [287]. Most recently, Kita and Toriumi [288] proposed that the $\mathrm{V}_{\mathrm{FB}}$ shifts correlate with the oxygen ion density in the various oxides.

These are all empirical models without any first principles modelling. In order to understand the atomic level mechanism, some periodic atomic models of the $\mathrm{SiO}_{2}-\mathrm{HfO}_{2}$ interface were constructed by Lin and Robertson [289,290], with substitutional $\mathrm{La}, \mathrm{Sr}, \mathrm{Al}$, or $\mathrm{Nb}$ ions. These are based on the model interfaces of Sharia [291] with the (100) face of $\beta$-cristobalite $\mathrm{SiO}_{2}$ matched to the (100) face of cubic $\mathrm{HfO}_{2}$, if the $\mathrm{HfO}_{2}$ lattice is rotated by $45^{\circ}$. The interface has no broken $\mathrm{Si}-\mathrm{O}$ bonds. A $\mathrm{HfO}_{2}$ layer then contains 4 oxygens per layer, a $\mathrm{SiO}_{2}$ layer contains 2 oxygen per layer, and the interfacial plane has 3 oxygens.

The $\mathrm{La}, \mathrm{Al}, \mathrm{Nb}, \mathrm{Sr}$ or $\mathrm{Mg}$ dopants are then introduced into this supercell by substituting at $\mathrm{Hf}$ sites, especially at the interfaces, as in fig 56. Charge neutrality is mainrained. For trivalent dopants ( $\mathrm{La}, \mathrm{Al}$ ), we introduce two La's and remove one oxygen from the $\mathrm{HfO}_{2}$ or $\mathrm{SiO}_{2}$ sub-layer. For divalent $\mathrm{Sr}$ or $\mathrm{Mg}$ dopants, we add one $\mathrm{Sr}$ and remove one oxygen. For pentavalent $\mathrm{Nb}$, we substitute two $\mathrm{Nb}$ for Hf's and add back an oxygen at the interface layer to make four oxygens, to balance charges (Fig 56(e).

The atomic positions are then relaxed by LDA total energy methods. The electronic structure is calculated and the valence band offsets (VBO) are derived from the local density of states of $\mathrm{HfO}_{2}$ and $\mathrm{SiO}_{2}$ layers away from the interface. The VBO is written as positive if the $\mathrm{HfO}_{2} \mathrm{VB}$ edge is deeper than $\mathrm{SiO}_{2}$ below the vacuum, to have the same sign convention as the flat band voltage.

Fig 56(a) shows the relaxed atomic structure of a pure $\mathrm{HfO}_{2}: \mathrm{SiO}_{2}$ interface. Fig 56(b) shows the structure of substitutional $\mathrm{La}_{\mathrm{Hf}}$ sites at the $\mathrm{HfO}_{2}: \mathrm{SiO}_{2}$ interface. Their local atomic structure has relaxes so 
that La sites become 6-fold bonded. Substitutional $\mathrm{Al}_{\mathrm{Hf}}$ sites relax to become 4-fold coordinated, as in amorphous $\mathrm{Al}_{2} \mathrm{O}_{3}$ (Fig 57c). Interfacial substitutional $\mathrm{Sr}_{\mathrm{Hf}}$ becomes 6-fold coordinated.

Fig 57(a) plots the VBOs of cells with various substitutional dopants at interfacial sites against the experimental $\mathrm{V}_{\mathrm{fb}}$ shifts for $\mathrm{Sr}$ and $\mathrm{La}$ oxides from Kirsch [285], for $\mathrm{Al}_{2} \mathrm{O}_{3}$ from Linder [292], and the positive shift of $\mathrm{Nb}$, compared to the base case of the pure $\mathrm{HfO}_{2}: \mathrm{SiO}_{2}$ system. We see that the shift direction is proportional to the experimental shift in each case. We find that the VBO does vary with the separation of the dopant and the vacancy, but this effect is much smaller than the main shift. Thus, the model reproduces the observed chemical trends.

Fig 57(b) plots the VBO shift versus the dopant valence. The calculated VBOs and experiment are similar for some cases. However, the trivalent dopants $\mathrm{La}$ and $\mathrm{Al}$ both introduce $\mathrm{O}$ vacancies but the calculated shifts are in opposite directions. On the other hand, trivalent $\mathrm{Al}$ and pentavalent $\mathrm{Nb}$ shift in the same direction. This argues against the vacancy model of Guha [277].

Fig 57(c) plots the calculated VBO shift against the work function of the dopant's parent metal, which is effectively its electronegativity. We see that the VBO shift varies monotonically with the dopant work function. Note that the shift of $\mathrm{La}$ and $\mathrm{Al}$ are in the opposite directions, consistent with La having a lower WF than $\mathrm{Hf}$ and $\mathrm{Al}$ having a higher WF. On the other hand $\mathrm{Al}$ and $\mathrm{Nb}$ have a calculated shift in the same direction, despite their different valences, but consistent with both having similar WFs, both higher than Hf. Note that the $\mathrm{V}_{\mathrm{fb}}$ shift of $\mathrm{Sr}$ is larger than that of La, consistent with the calculated VBO shift, and with the lower work function of $\mathrm{Sr}$ than La.

Hence, the VBO shift correlates with dopant electronegativity/work function, not with its valence. Our supercells have the same oxygen ion density, by construction, but the calculated VBOs differ greatly. Thus, the VBO does not correlate with oxygen ion density [288]. Hence, our models exactly reproduce the experimental trends of $\mathrm{V}_{\mathrm{fb}}$ and allow us to eliminate a number of models.

These results still need a simple explanation $[289,290]$. Is the dipole due to bulk bonds or interface bonds? There are two arguments against a bulk dipole model. First, if the bulk is represented by layers AB-A-B..., the dipole $\mathrm{A}^{+}-\mathrm{B}^{-}$on average cancels the dipole $\mathrm{B}^{-}-\mathrm{A}^{+}$, Fig 58(a), so that a network of bulk bonds does not create a net dipole. Second, a dipole is given by the product of charge and distance, $\mathrm{Q} \bullet \mathrm{d}$. but $\mathrm{Sr}$ gives a larger $\mathrm{V}_{\mathrm{FB}}$ shift than $\mathrm{La}$, although $\mathrm{Sr}$ is $2+$ while $\mathrm{La}$ is $3+$ and their ionic radii are quite similar. Thus, the effect must arise from an interface or graded interface not a bulk effect.

The shift arises simply because there is a large change in dielectric constant, $\mathrm{K}$, at the interface going from $\mathrm{HfO}_{2}$ to $\mathrm{SiO}_{2}$, Fig 58(b). The potential step due to a dipole is given by

$$
\Delta V=\frac{n Q d}{K}
$$

where $\mathrm{n}$ is the ion density, $\mathrm{Q}$ its charge, $\mathrm{d}$ the bond length, not just the basic dipole Q.d. In this case, a dipole $\mathrm{A}^{+}-\mathrm{B}^{-}$does not cancel the dipole $\mathrm{B}^{-}-\mathrm{A}^{+}$, as in the bulk, because each dipole is screened by a different $\mathrm{K}$ value.

The $\mathrm{V}_{\mathrm{fb}}$ shift between a pure $\mathrm{HfO}_{2}$ and a doped $\mathrm{HfO}_{2}$ is related to the change in mean electrostatic potential across the interface, and thus in the group electronegativity. This accounts for the trends in Fig 57(c). This gives an explicit atomic model of the flat band voltage shifts introduced by metal-oxide capping layers. The $\mathrm{V}_{\mathrm{fb}}$ shifts in the observed directions for all cases studied. The shifts correlate with the work function of the metal of the metal oxide, not with its valence or bond dipole.

\section{Electrical performace \\ 7.1 Mobility Degradation}

The objective of device scaling is to create smaller, faster devices. High speed requires high sourcedrain current, which in turn depends on the carrier mobility. Carriers in the FET behave like a twodimensional electron gas. The carrier density is determined by the vertical (gate) electric field which 
induces them. The carrier mobility in a $2 \mathrm{D}$ electron gas is found to depend in a 'universal' way on the gate field, according to a so-called 'universal mobility model', such as that by Takagi [293], in which the mobility of electrons and holes depends only on the effective gate field and the Si face, [100], [110] or [111]. The individual scattering processes $v_{\mathrm{i}}$ add up into a total scattering rate $v$,

$$
v=v_{1}+v_{2}+v_{3}
$$

so the processes limiting mobility add up according to Matthiessen's rule,

$$
\frac{1}{\mu}=\frac{1}{\mu_{C}}+\frac{1}{\mu_{P H}}+\frac{1}{\mu_{S R}}
$$

where $\mathrm{C}=$ Coulombic scattering, $\mathrm{PH}=$ phonon scattering, $\mathrm{SR}=$ interface roughness. The mobility is limited by different mechanisms at different gate fields, as in Fig 59. At low fields, the mobility is limited by Coulombic scattering by trapped charges in the oxide and/or interface; at moderate field it is mainly limited by phonon scattering, and at high fields it is mainly limited by interface roughness scattering. The different mechanisms also have different temperature dependences, with Coulomb scattering and roughness scattering being $\mathrm{T}$ independent.

CMOS devices with a $\mathrm{SiO}_{2}$ gate oxide have carrier mobilities close to the 'universal limit'. In that case, the mobility is limited mainly by the $\mathrm{Si}: \mathrm{SiO}_{2}$ interface roughness. The mobilities of high $\mathrm{K}$ gate oxide devices initially lay well below this universal curve, as seen by the data of Gusev [17], Ragnarsson [294], Guha [295], Hiratani [296], Torii [297] plotted in Fig 60, particularly for NMOS devices.

There are two main mechanisms of mobility lowering. The first mechanism is remote phonon scattering (RPS) by low energy polar phonon modes in the oxide by Fischetti [298], Fig 61. The second mechanism is remote Coulomb scattering (RCS) by trapped charge of Saito [299]. RCS is clearly important, as some high $\mathrm{K}$ oxides such as $\mathrm{Al}_{2} \mathrm{O}_{3}$ with no soft modes do show mobility degradation [17].

A lower nominal mobility might also arise from reduced carrier density, due to some of the induced charge being trapped at interface traps and not contributing to the mobile charge (Zhu [300]). This effect can be recognised by Hall measurements of mobility, where a direct decrease in Hall mobility can be seen in Ragnarrson [301].

Fischetti [298] noted that the high $\mathrm{K}$ of most oxides results from their low-lying polar vibration modes, the same modes that make a high $\mathrm{K}$. The oxides are incipient ferroelectrics and these soft polar modes would drive a ferroelectric instability if their frequency fell to zero. These polar modes have a strong coupling to carriers in the $\mathrm{Si}$ channel - hence 'remote scattering'. On the other hand, in $\mathrm{SiO}_{2}$ such modes have a much higher frequency and do not have a large coupling.

The importance of remote phonon scattering is that it is intrinsic and so it could be a fundamental limit to mobility in small EOT devices. On the other hand, remote Coulomb scattering (RCS) is an extrinsic effect, which one might hope to reduce by processing and reducing trap densities. RCS would be preferred technologically.

The two mechanisms can be distinguished by their temperature, field and thickness dependence. Phonon scattering is the only mechanism whose mobility decreases as the temperature is raised, because the phonon numbers increase with T. Surface roughness is independent of T, and mobility limited by Coulombic scattering can increase at higher temperatures, see Fig. 59. Ren [302] found there is indeed a T dependence of $1 /$ mobility in the mid-field range where it is expected.

A second method to distinguish the mechanisms is to plot the mobility against oxide thickness, as in the work of Ragnarsson [301], Gusev [17], Casse [303], Lee [12], Narayanan [304] and Ragnarsson [305]. In this case, a device is made with a variable thickness of ALD-grown $\mathrm{SiO}_{2}$ layer below a fixed

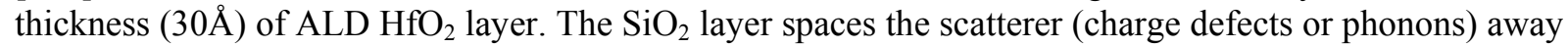
from the carriers. The mobility can be expressed as an 'additional inverse mobility' compared to the reference mobility for a gate stack with only the minimum $\mathrm{SiO}_{2}$ thickness, 


$$
\frac{1}{\mu_{\text {add }}}=\frac{1}{\mu_{\mathrm{HfO} 2}}-\frac{1}{\mu_{\text {ref }}}
$$

The extra inverse mobility is found to decrease exponentially with the $\mathrm{SiO}_{2}$ layer thickness, $\mathrm{t}$, (Fig 61) as

$$
\Delta\left(\frac{1}{\mu_{\text {add }}}\right)=\frac{1}{\mu_{0}} \exp (-2 \beta . t)
$$

where $\mu_{0}$ is a constant and $\beta$ equals the thermal wavevector, $\beta=(2 \mathrm{mkT})^{1 / 2} / \mathrm{h} . \mathrm{k}$ is Boltzman's constant. Interestingly, RPS and RCS from the $\mathrm{HfO}_{2}$ layer both decay with the same rate with $t$ (Fig 62). Casse [303] showed data for non-optimised samples with a sizable mobility reduction. Ragnarrson [305] gave data for highly scaled, scavenged gate stacks.

Generally, remote Coulomb scattering is dominant at lower fields and phonon scattering at moderate fields. The excess scattering was found to vary linearly with gate field at low gate fields for varying $\mathrm{SiO}_{2}$ thickness, Fig 63. This linear dependence is as expected for remote Coulomb scattering. This is strong evidence for RCS. The temperature dependence of the excess could also be derived. The total mobility plot was then re-assembled from the Mattheisen's rule and it was found to describe well the experimental data and its $\mathrm{T}$ dependence (Casse [303]). Thus, although the mobility is reduced at both low and medium fields, the main cause is RCS.

These observations have allowed firms to engineer a solution to the mobility problem, as seen in Fig 64. It is useful that there is an $\mathrm{SiO}_{2}$ layer between the channel and $\mathrm{HfO}_{2}$ layer to screen the remote scattering. The high $\mathrm{K}$ oxide layer should be thin, so that the defect density is low, and there are few traps Narayanan [304] optimised the annealing and nitrogen content of the $\mathrm{HfO}_{2}$ interface to obtain the best mobility vs. $\mathrm{T}_{\text {inv }}$ performance. Their data in the upper part of Fig 64 now approaches the RPS limit, and is not so far below the universal mobility model limit.

The data was then extended by data from scavenged gate stacks, by Ragnarrson [305]. The interfacial oxide is of high quality, and the data is seen to follow the same trend. The mobility decreases as $t_{\text {inv }}$ declines, and it is bounded by the theory curve (Fig 64).

\subsection{Reliability}

Generally speaking, the reliability for $\mathrm{HfO}_{2}$ gate stacks arises from trapping at existing defects rather than the creation of additional defects [217]. Novel pulsed measurement methods have ben developed to speed up data collection [306].

There are two basic instabilties, negative bias stress instability (NBTI) which occurs in nFETs and positive bias stress instability (PBTI) which occurs in pFETs. In nFET and PBTI, the gate voltage is positive, and electrons are injected from the $\mathrm{Si}$ channel into the gate oxide at the energy of the $\mathrm{Si}$ conduction band. At this energy, the electrons are injected into the $\mathrm{V}^{0}$ state of the $\mathrm{O}$ vacancy, Fig 65 $[218,307]$. This causes $\mathrm{V}_{\mathrm{t}}$ to shift as a power law with time,

$$
\Delta \mathrm{V}_{\mathrm{t}}=\mathrm{At}^{\mathrm{n}}
$$

where the exponent $\mathrm{n} \sim 0.15$ to 0.2 . This mechanism causes a large increase in PBTI compared to a reference SiON dielectric, Fig 66.

On the other hand, in pFET and under NBTI, the gate is sent negative, and holes are injected into the oxide valence band. There is no particular hole trap in $\mathrm{HfO}_{2}$ at this energy, and the holes become trapped at defects in the interfacial $\mathrm{SiO}_{2}$ layer, Fig 65, causing NBTI [295]. Again, the $\mathrm{V}_{t}$ shifts follows the same power law as PBTI. In this case, the mechanism involves trapping in $\mathrm{SiO}_{2}$ so the size of NBTI is not much larger than for the reference SiON case. This is shown schematically in Fig. 66. 
The size of NBTI and PBTI both depend strongly on the thickness of the $\mathrm{SiO}_{2}$ interlayer. Therefore NBTI and PBTI both increase strongly as the EOT is reduced by scavenging. Fig 67 shows a normalized version after Cartier [307]. The slope is seen to be very high. An unnormalised version is given by Ragnarrson [305], Fig 68. Fig 68 plots the gate bias voltage that would cause a $30 \mathrm{mV}$ total PBTI or NBTI shift over a period of 10 years, the required lifetime. Thus, a small gate voltage implies an unstable device. This shows that NBTI does not allow EOT to be decreased below $6.5 \mathrm{~A}$, on this basis. This makes reliability the limiting feature of small $t_{\text {inv }}$ values. Thus, minimum oxide thickness does depend ultimately on reliability, just as in the 'Doomsday plot', but in a different era.

There are certain engineering solutions to improve the PBTI and NBTI reliability margins. PBTI due to electron injection into the $\mathrm{O}$ vacancy level can be reduced by removing the vacancy level by adding some nitrogen [305] or La [308]. As described in the defect section 5.3, $\mathrm{N}$ or La next to an $\mathrm{O}$ vacancy will repel the vacancy level to higher energies in the gap, so that electrons are no longer injected into that level.

NBTI can be reduced by band engineering of the channel band line-up. The exact nature of the hole trap in NBTI is not well known. Therefore it is not possible to passivate it. On the other hand, Franco $[309,310]$ have found that in SiGe devices, the Ge-Si band offset can be used to cover up the defect level, to mitigate this trapping.

\subsection{Scavenging and ultra-low EOT}

The continued scaling of EOT below $1.0 \mathrm{~nm}$ and towards $0.5 \mathrm{~nm}$ requires us to either reduce the thickness of the interfacial layer, or increase the $\mathrm{K}$ values. In 2006-2010 there was considerable work towards developing oxides with higher $\mathrm{K}$ than $\mathrm{HfO}_{2}$, as described in section 4.3. However, Ando [91] pointed out that the $\mathrm{SiO}_{2}$ based interlayer makes the dominant contribution to EOT so that a higher $\mathrm{K}$ would have little effect. To solve this problem Ando [95] suggested increasing the $\mathrm{K}$ of the interlayer, for example by alloying it with lanthanides. However, there are disadvantages to adding La, as it affects the gate threshold voltage. These layers had an undesired effect on carrier mobility.

The remaining method was to reduce the interfacial $\mathrm{SiO}_{2}$ layer thickness. The $\mathrm{SiO}_{2}$ layer can be thinned by the oxide scavenging process, in which an electropositive metal is placed in the gate to extract $\mathrm{O}$ from the $\mathrm{SiO}_{2}$ by diffusion through the $\mathrm{HfO}_{2}$ layer, Fig 69. This reduces the $\mathrm{SiO}_{2}$ back to Si. Fig 70 shows a TEM cross section of the gate stack after scavenging. This process requires that the heat of formation per $\mathrm{O}$ atom of the metal's oxide is greater than that of $\mathrm{SiO}_{2}$ (Fig 71). Ando [95] and Frank [97] have carried out extensive work on this process. Fig 71 shows that $\mathrm{W}$ or Ta will not scavenge from $\mathrm{SiO}_{2}$ but that $\mathrm{Ti}$, $\mathrm{Hf}$ and $\mathrm{La}$ will do so. $\mathrm{Ti}$ is arguably the most suitable because the heat of formation of $\mathrm{TiO}_{2}$ is larger than $\mathrm{SiO}_{2}$ but not too large, and TiN has a mid gap work function whixch is also useful.

The scavenging process can be arranged so that the scavenging metal is placed in various locations, as shown in Fig 69. The temperature dependence of the scavenging effect depends on the oxgygen diffusion through $\mathrm{HfO}_{2}$, as shown in Fig 72. The onset is at 450-550 C. Scavenging can be used in both gate first and gate last processes, by Ando [95] and Ragnarsson [305]. Scavenging can even be used in conjunction oxide capping layers setting the gate work function, in gate last process, with appropriate choice of anneal temperature to allow diffusion of both $\mathrm{O}$ and the cation. The electron mobility in scavenged stacks (Fig 73) follows a similar curve to that shown previously [94], fig 64.

The importance of the gate last process is that it enables the use of much lower process temperatures for the oxide. Given that scavenging can still be used for gate last, it is important to understand the degree of $\mathrm{V}_{t}$ control that can be achieved [311-313]. In particularly, there is the problem of $\mathrm{V}_{\mathrm{t}}$ roll-off for the pFET [275]. Fig 74 plots the $\mathrm{V}_{\mathrm{t}} \mathrm{vs}$ EOT for gate last stacks, including the scavenged regime [312]. We see that the $V_{t}$ 's are not as close to the band edges in the linear regime as in Fig. 52. We see that $V_{t}$ is constant down to lowest EOT values for nFET. For pFET, we see that $V_{t}$ is constant down to $\mathrm{EOT}=8 \AA$, and then turns down sharply, as the roll-off starts. It is notable that this critical thickness of $0.8 \mathrm{~nm}$ is much lower than the $2.5 \mathrm{~nm}$ for gate-first in Fig 52. 
The net result is that scaling can continue to very small effective oxide thicknesses, as summarised in the Moore's Law plot in fig 75.

With the development of FinFET type device geometries, the need to use near band edge gate work functions is relaxed. It is possible to use $\mathrm{TiN}$ and $\mathrm{TiON}_{\mathrm{x}}$ drived from oxidised $\mathrm{TiN}$ as the n-type and ptype metal gates instead, Fig 76 [97].

\section{$8 \quad$ Ge Oxides \\ 8.1 $\mathrm{Ge} / \mathrm{GeO}_{2}$ interface}

Germanium was employed for the original transistor, but was then abandoned in favor of Si due to its small band gap [314]. There has recently been a resurgence of interest in Ge [315-321], primarily due to its high injection velocity needed for continued scaling of CMOS.

Of the possible alternative semiconductors to $\mathrm{Si}$, Ge stands out as a solution for both higher electron and hole mobilities. Ge has the highest hole mobility of any tetrahedral semiconductor, much higher than of $\mathrm{Si}$ (Table 4). However, the band gap of its native oxide is $33 \%$ lower and the bond energy of Ge-O bonds is over $35 \%$ lower than that of Si-O bonds. The $\mathrm{Ge}^{2+}$ state has higher stability than the equivalent $\mathrm{Si}^{2+}$ state. The charge neutrality level of Ge lies close to its valence band top, rather than $30 \%$ up the gap as in Si. Each of these aspects causes less easy device processing.

For devices, the performances of Ge pFETs are very good, with on-currents corresponding to the high hole mobilities. However, until recently, nFETs had poorer performances, not reflecting the high electron mobility, largely due to the higher $\mathrm{D}_{\mathrm{it}}$ in the upper band gap [322,323]. Overall, $\mathrm{D}_{\mathrm{it}}$ is an order of magnitude higher than for $\mathrm{SiO}_{2}$ [321]. Thus we must understand the Ge interfaces better.

There are some well known problems with the $\mathrm{Ge}: \mathrm{GeO}_{2}$ system. The native oxide of $\mathrm{Ge}$ is hygroscopic. Second, $\mathrm{GeO}_{2}$ is more defective, and the $\mathrm{Ge} / \mathrm{GeO}_{2}$ system had a much worse interface in $\mathrm{CV}$ plots [324]. However, if $\mathrm{GeO}_{2}$ is deposited on $\mathrm{SiO}_{2}$ and not in direct contact with $\mathrm{Ge}$ itself, then $\mathrm{GeO}_{2}$ is a good insulator with few defects [325]. The problem with the Ge: $\mathrm{GeO}_{2}$ system is the interface, not the $\mathrm{GeO}_{2}$. The contact with $\mathrm{Ge}$ acts as a constant source of $\mathrm{Ge}$, and under annealing, $\mathrm{Ge}$ and $\mathrm{GeO}_{2}$ react to form volatile $\mathrm{GeO}$ molecules.

$$
\mathrm{Ge}+\mathrm{GeO}_{2}=2 \mathrm{GeO} \uparrow
$$

These problems lead to the formation of interface gap states. $\mathrm{GeO}$ formation can be suppressed by using a high pressure of $\mathrm{O}_{2}$, as noted by Toriumi [326,327], and Kuzum [328].

The abruptness of the $\mathrm{Si} / \mathrm{SiO} 2$ interface is seen by the low density of intermediate oxidation states between $\mathrm{Si}$ and $\mathrm{Si}^{4+}$ in the XPS core level spectra by Himpsel [56]. Keister [57] showed that the $\mathrm{SiO}_{\mathrm{x}}$ phases at a non-abrup $\mathrm{Si} / \mathrm{SiO}_{2}$ interface would disproportionate into $\mathrm{Si}$ and $\mathrm{SiO}_{2}$ giving a more abrupt interface.

Nevertheless, the Ge: $\mathrm{GeO}_{2}$ interface can be flat and abrupt, as shown by the TEM cross sectional images and core level XPS data of Sasada [329], Fig 77. Secondly, Delabie [330] showed that the $\mathrm{Ge} / \mathrm{GeO}_{2}$ interface prepared in an optimum way can have a reasonanlby low $\mathrm{D}_{\mathrm{it}}$ density, reminiscent of the desire for a $\mathrm{SiO}_{2}$ interfacial layer below $\mathrm{HfO}_{2}$ on $\mathrm{Si}$.

The thermal stability of $\mathrm{GeO}_{2}$ has been examined in detail in the context of the gate stack structure. The mechanism of $\mathrm{GeO}$ evolution from the $\mathrm{GeO}_{2} / \mathrm{Ge}$ interface was studied by $\mathrm{Ge}$ and $\mathrm{O}$ isotopic labeling and thermal desorption spectroscopy methods by Kita [331,332]. It showed that $\mathrm{O}$ vacancies were the mobile species in $\mathrm{GeO}_{2}$. The $\mathrm{O}$ vacancies are created by the interfacial reaction at the $\mathrm{Ge} / \mathrm{GeO}_{2}$ interface, the $\mathrm{O}$ vacancy diffuses to the top surface, and then it reacts with $\mathrm{GeO}_{2}$ unit to evolve as an $\mathrm{GeO}$ molecule, as summarised in Fig 78. It is not like a Deal-Grove process where an interstitial molecule diffuses through the oxide.

Hence, $\mathrm{GeO}$ formation must be supressed for better performance. This can be controlled through the use of high-pressure oxidation of $\mathrm{Ge}\left(>70 \mathrm{~atm} . \mathrm{O}_{2}\right)$ followed by a lower temperature oxidation step [333]. It was recently shown that alloying an oxide of electropositive metal such as $\mathrm{Y}$ increases the 
formation energy of the $\mathrm{O}$ vacancy, and thus reduces the diffusion rate of $\mathrm{O}$ vacancies in $\mathrm{GeO}_{2}$. This reduces the etch rate of the $\mathrm{GeO}_{2}$ and the volatilisation rate of $\mathrm{GeO}$ [334].

The band offsets for various $\mathrm{Hf}$ and $\mathrm{Zr}$ oxides on $\mathrm{Ge}$ have been measured by internal photoemission, separating out the band offsets of the $\mathrm{GeO}_{2}$ interfacial layer by Afanasev $[335,336]$ and by XPS by Ohta [337]. The offsets are quite similar to those on $\mathrm{Si}$, the $\mathrm{CB}$ offset being higher by $0.1 \mathrm{eV}$. A problem for $\mathrm{Ge}$ is that $\mathrm{GeO}_{2}$ has a much smaller $\mathrm{CB}$ offset than $\mathrm{SiO}_{2}$, while the $\mathrm{VB}$ offset is similar to that of $\mathrm{SiO}_{2}$ on $\mathrm{Si}[338,339]$, Fig 79. This arises because the band gap of $\mathrm{GeO}_{2}$ is much smaller, while the $\mathrm{VB}$ top of $\mathrm{GeO}_{2}$ and $\mathrm{SiO}_{2}$ consists of $\mathrm{O} 2 \mathrm{p}$ states, so the $\mathrm{VB}$ offset is similar in both cases.

\subsection{Ge dangling bond}

A problem with Ge interfaces is that the Fermi energy is often pinned towards the bottom of the gap, and so it is difficult to invert from p-type to n-type [340]. The CV plots can appear poor. The low pinning energy has been attributed to the lower energy of the Ge dangling bond (DB) level than in $\mathrm{Si}$ [341], or equivalently to the low CNL energy. We recall that the DB lies near midgap in $\mathrm{Si}$, and gives rise to two $\mathrm{D}_{\text {it }}$ peaks corresponding to its $+/ 0$ and $0 /-$ transitions [342]. Weber [341] suggested that the neutral Ge DB level actually lies below the Ge valence band top, so that it is always in its negative configuration. In addition they suggest that the hydrogen interstitial level is also below the Ge VB top, so that hydrogen is also negative, and thus $\mathrm{Ge}^{-}$and $\mathrm{H}^{-}$will repel each other and prevent atomic hydrogen from passivating the Ge DB and forming a Ge-H bond. However, Broqvist [343] showed that Ge DB level lies low in the band gap, but just above the Ge VB top, and this DB should be observable. Note that the Fermi level at the Ge-metal interface is pinned low in the Ge gap [344,345], but the relevant question is where it is pinned at the Ge-oxide interface.

A further question about the $\mathrm{Ge} / \mathrm{GeO}_{2}$ interface is that the chemical identity of the interface states is not known. For $\mathrm{Si} / \mathrm{SiO}_{2}$ interfaces, Brower [346] could identify the $\mathrm{P}_{\mathrm{b}}$ center as a $\mathrm{Si} \mathrm{DB}$ from its ESR hyperfine spectrum.

In Ge, the interfacial Ge DB level (or Ge $\mathrm{P}_{b}$ centre) is difficult to observe by ESR. Houssa [347] suggested that the Ge $\mathrm{P}_{\mathrm{b}}$ centre is less readily observed because of thermal effects, Fig 80 . The temperature of viscous flow relaxation of $\mathrm{GeO}_{2}$ is much lower than for $\mathrm{SiO}_{2}$, so that annealing is presently carried out at a slightly too high temperature to observe $\mathrm{Ge}_{\mathrm{b}}$. The $\mathrm{GeO}_{2}$ network has relaxed sufficiently that the $\mathrm{Ge} \mathrm{P}_{\mathrm{b}}$ centers have been annealed away.

Recently, Stesmans et al [348,349] have observed the Ge $\mathrm{P}_{\mathrm{b}}$ state center by ESR in Ge-Si alloys, in the $60-80 \%$ Ge composition range. Baldovino [350,351] have observed the Ge $\mathrm{P}_{\mathrm{b}}$ by optical detected magnetic resonance at $\mathrm{Ge} / \mathrm{GeO}_{2}$ interfaces. Thus, the Ge DB does exist in the gap. The Ge DB signal has finally been observed at a defective $\mathrm{Ge}: \mathrm{GeO}_{2}$ interfaces by Paleari [352] using electrically detected magnetic resonance, Fig 81. It shows the angular response expected for a $\mathrm{P}_{\mathrm{b}}$ center. Recently, the reaction of hydrogen with the Ge $\mathrm{P}_{b}$ center has been observed by Stesmans [353]. The difficulty of passivating the Ge DB arises from the distribution of activation energies for the passivation and depassivation reactions, so that only $60 \%$ of Ge $\mathrm{P}_{\mathrm{b}}$ 's are passivated in typical conditions, whereas $99.98 \%$ of $\mathrm{Si} \mathrm{P}_{\mathrm{b}}$ 's are passivated for the $\mathrm{Si} / \mathrm{SiO}_{2}$ interface, because of a more favourable set of kinetic parameters. If the Ge parameter distribution could be narrowed, then the Ge system could also be better passivated (eg by flatter interfaces).

The structure of atomic defects at the $\mathrm{Ge}: \mathrm{GeO}_{2}$ interface may be more complicated than at the $\mathrm{Si}: \mathrm{SiO}_{2}$ interface. The simplest defect is the $\mathrm{O}$ vacancy. But this defect can also re-arrange to form a valence alternation defect, with a 3-fold Ge site and a 3-fold $\mathrm{O}$ site, as noted by Broqvist [354] and $\mathrm{Li}$ [355]. Both these defect sites give gap states. The 3 -fold Ge site gives a state just below the Ge VB edge, and the 3 -fold $\mathrm{O}$ site gives a state localised on adjacent Ge sites that lies just above the Ge conduction band, as shown in Fig 82. Zhang [361] has noticed that carrier localisation in these resonant states lowers the field effect mobility in nFET and pFET below there expected values.

\subsection{ALD on Ge}


The study of ALD high-k dielectrics on Ge has been underway for several years [356]. It is found that direct contact of Ge with a high-k dielectric results in inferior electrical behavior due to interface state generation resulting in mobility degradation [357,358]. As a result, Caymax [359] investigated using a thin $\mathrm{SiO}_{2}(\mathrm{k}=3.9), \mathrm{GeO}_{2}(\mathrm{~K}=5.2-5.8)$ or $\mathrm{GeON}(\mathrm{K} \sim 6.0)$ layer between the high-K and $\mathrm{Ge}$ substrate. From a scaling perspective, it would be desirable to minimize a low-K dielectric in the gate stack [2], and so the most recent work has emphasized avoiding the introduction of a thin Si layer at the interface resulting in relatively low- $\mathrm{K} \mathrm{SiO}_{2}$ formation.

Recent studies of an ALD process on the $\mathrm{GeO}_{x}$ surface have shown that the various oxidation states of Ge undergo a reaction with trimethyl-aluminum (TMA) [360]. This so-called "clean-up" effect, observed originally on III-V substrates, severely reduces surface oxides. As seen in Fig. 83, a Ge(100) surface etched with de-ionized water results in a surface with various Ge oxidation states. Exposing this surface to the ALD reactor vacuum ambient causes further surface oxidation. However, the oxides are immediately reduced upon exposure to the first ALD precursor pulse, TMA. Subsequent water and TMA exposures result in a gradual reduction of the $\mathrm{GeO}_{\mathrm{x}}$. Similar results are seen for a clean Ge surface, where the ALD vacuum ambient causes surface oxide formation again (Fig. 83(b)). These results demonstrate the power of in-situ studies vs. ex-situ analysis, and furthermore present a contrast to entirely UHV studies, where the initial oxidation always present in a commercial ALD tool is absent.

Fig 83 shows the corresponding ex-situ XPS of the interface formed from the post-ALD plasma oxidation process with substantial concentrations of the $\mathrm{Ge}^{+3}$ oxidation state observed. A correlation of the extracted $D_{i t}$ with $\mathrm{GeO}_{\mathrm{x}}$ thickness indicates the $\mathrm{Ge}^{+3}$ state is correlated with lower $D_{i t}$ values and thus better interface passivation (Fig. 83(b)). A minimum $\mathrm{GeO}_{\mathrm{x}}$ thickness for sufficient device passivation of $0.5 \mathrm{~nm}$ is also noted. Other work by Zhang et al [361-364] examining the effects of plasma oxidation of the Ge surface prior to ALD indicate a somewhat higher $D_{i t}$ level is detected, particularly as the $\mathrm{GeO}_{\mathrm{x}}$ is thinned below $2.7 \mathrm{~nm}$. This degradation was attributed to "ALD damage", i.e. reactions of the $\mathrm{GeO}_{\mathrm{x}}$ layer during the subsequent ALD process.

An alternative approach for $\mathrm{Ge}$ interface passivation is to use ozone oxidation at $400^{\circ} \mathrm{C}$ prior to ALD of $\mathrm{Al}_{2} \mathrm{O}_{3}$ [363].

Alternative interfacial layers have also been investigated previously, such as $\mathrm{GeON}$ integrated with high-k dielectrics [364-367]. Fig. 83(c) shows that in-situ studies of the GeON/Ge interface is relatively robust compared to the $\mathrm{GeO}_{2} / \mathrm{Ge}$ interface as far as reactions with the TMA precursor are concerned [358]. It is seen that the interfacial chemistry is minimally affected upon exposure to the TMA $/ \mathrm{H}_{2} \mathrm{O}$ process and likely due to the strong oxynitride bonding. However, electrical characterization indicates that a somewhat higher $D_{i t}$ for an ALD $\mathrm{HfO}_{2}$ dielectric on GeON after a forming gas anneal $\left(370{ }^{\circ} \mathrm{C}, 30 \mathrm{~min}\right.$.) was recently reported [367], compared to that noted above for a $\mathrm{GeO}_{\mathrm{x}}$ interface with $\mathrm{Al}_{2} \mathrm{O}_{3}$. Therefore, recent work appears to emphasize work on engineered $\mathrm{GeO}_{2}$ as noted above, with the ALD high-k dielectric in place before exposure to the post-deposition oxidation process. Sulfur passivation of $\mathrm{Ge}$ using $\mathrm{H}_{2} \mathrm{~S}$ in conjunction with ALD is also a useful factor [368-371].

Recently, fundamental studies of Al ALD on atomically clean Ge has been investigated by Kummel [372] using in-situ dosing, STM and XPS analysis in conjunction with first-principles calculations. TMA was exposed to the Ge surface at $5 \times 10^{-9}$ Torr at room temperature to result in a nearsaturation exposure of $10000 \mathrm{~L}$, resulting in a coverage of 0.8 monolayers (ML) of the adsorbed species according to STM imaging. The actual coverage of Al on the surface is estimated to be $\sim 20 \%$ of the surface, based on in-situ XPS measurements, and likely results from steric effects of the methyl ligands. Some ordering of the adsorbed species is observed by STM, and a dissociation of TMA to form a dimethyl-Al (DMA) and mono-methyl (MMA) complex on the Ge dimer is posited to be the stable structure at room temperature. Subsequent annealing of the TMA dosed surface to $250^{\circ} \mathrm{C}$ under UHV results in a decrease in surface carbon concentration while the $\mathrm{Al}$ surface concentration remains constant, with the formation of volatile $\mathrm{C}_{2} \mathrm{H}_{6}$ and $\mathrm{CH}_{4}$ species suggested as the principle reaction channels. An adsorbed species coverage of $0.4 \mathrm{ML}$ is reported. Further annealing to $450^{\circ} \mathrm{C}$ under UHV again results in 
a further reduction in the detected $\mathrm{C}$ surface concentration to $\sim 20 \%$, while the $\mathrm{Al}$ concentration remained constant at $\sim 20 \%$, and an adsorbed species coverage of $0.15 \mathrm{ML}$. The remaining $\mathrm{C}$ is suggested to be present as remaining DMA and MMA species.

In comparison to reactions from using a standard ALD tool, where the precursor is entrained in flowing $\mathrm{N}_{2}$, reactor pressures $\sim 7$ Torr are utilized with pulse times $\sim 0.1 \mathrm{~s}$, corresponding to exposures of the TMA (and $\mathrm{H}_{2} \mathrm{O}$ ) on the order of $10^{6} \mathrm{~L}$ are employed [358]. In addition, the Ge surface is not typically atomically clean, but likely has a native or chemical oxide on the surface initially (or upon exposure to the ALD reactor ambient), as seen in Fig. 84. So the correlation to the reactions under actual ALD conditions remain to be mapped out, although such UHV studies certainly points toward important reaction channels. In this regard, Lee et al [372] have examined Ge surfaces saturated with $\mathrm{H}_{2} \mathrm{O}\left(\sim 10^{6} \mathrm{~L}\right)$ followed by TMA exposure $(\sim 60,000 \mathrm{~L})$. An enhanced reaction of the TMA with the hydroxylated surface is observed, as expected, with somewhat higher Al concentrations $(\sim 40 \%)$ detected on the surface.

\section{Advanced Ge stacks and Device performance}

A way to minimise the $\mathrm{O}$ diffusion problem that creates interfacial defects is to use a very thin $\mathrm{Al}_{2} \mathrm{O}_{3}$ diffusion barrier layer. A good $\mathrm{Ge} / \mathrm{GeO}_{2}$ interface has perhaps given the lowest interface state density. A very thin $\mathrm{Al}_{2} \mathrm{O}_{3}$ layer is deposited on top of this. $\mathrm{O}$ diffusion through the $\mathrm{Al}_{2} \mathrm{O}_{3}$ is small so this suppresse s any outward $\mathrm{O}$ vacancy diffusion that would cause an interfacial reaction [374]. Zhang [359361] created this type of stack by depositing $\mathrm{Al}_{2} \mathrm{O}_{3}$ and then sub-cutaneously oxiding the $\mathrm{Ge}$ by a room temperature oxygen plasma (Fig 85). This appears to provide substantial passivation of interfacial defects, and promising electrical results. A reduction of the interface state density $\left(D_{i t}\right)$ from the $10^{12} \mathrm{~cm}^{-2} \mathrm{eV}^{-1}$ to as low as $5 \times 10^{10} \mathrm{~cm}^{-2} \mathrm{eV}^{-1}$ have been reported for capacitors. $\mathrm{HfO}_{2}$ can be deposited on top of this to complete the gate stack if necessary. A thicker $\mathrm{Al}_{2} \mathrm{O}_{3}$ layer is to be avoided as this has a higher defect density. Han [380] has found that a nitrogen plasma on the $\mathrm{Al}_{2} \mathrm{O}_{3}$ can further lower $\mathrm{D}_{\text {it }}$.

Fig 86 compares Ge MOSFET field effect mobilties. Shang [320] achieved an electron mobility of $100 \mathrm{~cm} 2 / \mathrm{V}$.s for $8 \mathrm{~nm}$ EOT in 2004. Toriumi used the high pressure/lowe pressure annealing technique to inhibit interface defects and achieved an electron mobility of $1100 \mathrm{~cm} 2 / \mathrm{V} . \mathrm{s}$ in 2009 for an unknown EOT. The $\mathrm{Al}_{2} \mathrm{O}_{3}$ diffusion barrier method allowed Zhang [359] to achieve electron mobilities of up to 937 $\mathrm{cm} 2 / \mathrm{V} . \mathrm{s}$ on EOTs of $1.1 \mathrm{~nm}$ and electron mobility of $754 \mathrm{~cm} 2 / \mathrm{V} . \mathrm{s}$ for an EOT of $0.82 \mathrm{~nm}$.

This approach has allowed a reduction of the interface state density $\left(D_{i t}\right)$ from the $10^{12} \mathrm{~cm}^{-2} \mathrm{eV}^{-1}$ to as low as $5 \times 10^{10} \mathrm{~cm}^{-2} \mathrm{eV}^{-1}$ to be reported for capacitors, a corresponding peak mobility of $437 \mathrm{~cm}^{2} / \mathrm{Vs}$ was reported for pMOS transistors fabricated on $\mathrm{Ge}(100)$ [361].

It is noted that high inversion carrier concentration (high field) mobilities are also substantially (x2) higher than the unoxidized $\mathrm{Al}_{2} \mathrm{O}_{3} / \mathrm{Ge}$ stack, and much higher $(\sim 4 \times)$ that for the $\mathrm{Si}$ "universal" mobility curve. Further work on nMOS Ge devices also appears promising, with peak mobilities of 937 $\mathrm{cm}^{2} /$ Vs reported [359].

These results generally indicate that an engineered $\mathrm{GeO}_{2}$ layer, with attention to the sequence of the plasma oxidation process, may provide a useful route to controlling the interfacial defect states. Further work is needed to optimize the gate dielectric properties, as border traps are detected in the films. Interfaces between the dielectrics (e.g. $\mathrm{GeO}_{2} / \mathrm{Al}_{2} \mathrm{O}_{3}$ ) appear to be a focus of this research as well.

\section{8}

\section{Summary}

This paper has reviewed the materials chemistry, bonding and electrical behaviour of oxides needed to replace $\mathrm{SiO}_{2}$ as the gate oxide in CMOS devices. The new oxides must satisfy six conditions to be acceptable as gate dielectrics, a high enough $\mathrm{K}$ value, thermal stability, kinetic stability, band offsets, good interface quality with $\mathrm{Si}$, and low bulk defect density. $\mathrm{HfO}_{2}$ and $\mathrm{Hf}$ silicate have emerged as the preferred oxides. The necessary deposition and processing to produce working devices has been achieved. However, the oxides need to be optimised substantially further, in order to achieve high performance devices. This requires improvement of flat band voltage and lower defect densities. The main defects in 
the oxides are oxygen vacancies and interstitials. Oxygen vacancies are the largest problem as they give rise to defect levels close to the $\mathrm{Si}$ conduction band. Flat band voltage instability is due to electron trapping at the $\mathrm{O}$ vacancy. Mobility degradation is largely due to remote charge scattering by charged defects or soft phonons in the oxide. DC flat band voltage shifts tend to be due to interaction and reaction of the gate electrode and the high $\mathrm{K}$ oxide.

\section{Acknowledgements}

The authors would like to thank K Xiong, K Y Tse, L Lin for many calculations, P MnIntyre and S Stemmer for illustrations, V Afanasev, G Bersuker, E Cartier, L Colombo, M Copel, S Takagi, and A Toriumi for valuable discussions. RMW acknoewledges an IBM Faculty award and JR acknowledges funding from EPSRC. 


\section{References}

1. M T Bohr, R S Chau, T Ghani, K Mistry,' The high-k solution', IEEE Spectrum 4429 (2007)

2. $\quad$ G Wilk, R M Wallace, J M Anthony, J App Phys 895243 (2001)

3. A I Kingon, A I Kingon, J P Maria, S K Streiffer, Nature 4061032 (2000)

4. R M Wallace, G Wilk, eds, MRS Bulletin 27 (March 2002)

5. J Robertson, Euro Phys J Appl Phys 28265 (2004)

6. M Houssa, 'High K Gate oxides', IOP, London (2003)

7. A A Demkov, A Navrotsky, 'Gate oxides', Kluwer, Amsterdam (2004)

8. H Huff, D Gilmer, 'Gate oxides', Springer, Berlin, (2004)

9. J Robertson, Rep Prog Phys 69327 (2006)

10. E P Gusev, V Narayanan, M M Frank, IBM J Res Dev 50387 (2006)

11. M leong, V Narayanan, D Singh, A Topol, V Chan, Z Ren, Materials Today 926 (June 2006)

12. B H Lee, J Oh, H H Tseng, R Jammy, H Huff, Materials Today 932 (June 2006)

13. B H Lee, S C Song, P Kirsch, IEEE Trans ED 558 (2008)

14. S H Lo, D A Buchanan, Y Taur, W Wang, IEEE ED Lett 18209 (1997)

15. K Mistry, C Allen, C Auth, B Beattie, D Bergstrom, M Bost, M Buehler, A Cappelani, R Chau, C H Choi, G Ding, K Fischer, T Ghani, R Grover, W Han, D Hanken, M Hattendorf, J He, J Hicks, D Huessner, D Ingerley, P Jain, R James, L Jong, S Joshi, C Kenyon, K Kuhn, K Lee, H Liy, B McIntyre, P Moon, J Neiynck, C Parker, D Parsons, L Pipes, M Prince, P Ranade, T Reynolds, J Sandford, L Schifren, J Sebastian, J Seiple, D Simon, S Sivakumar, P Smith, C Thomas, T Troeger, P Vanderyoon, S Williams, K Zawadzi, IEEE IEDM Tech Digest (2007) p10.2;

16. M Chudzik, B Doris, R Mo, J Sleight, E Cartier, C Dewan, D Park, H Bu, W Natzle, W Yan, .. M Khae, T C Chen, Tech Digest VLSI (IEEE, 2007) p194

17. E P Gusev, D A Buchanan, E Cartier, A Kumar, S Guha, A Callegari, S Zafar, P C Jamison, D A Neumayer, M Copel, M A Gribelyuk, H Okorn-Schmidt, C D’Emic, P Kozlowski, K Chan, N Bojarczuk, L A Ragnarsson, P Ronsheim, K Rim, R J Fleming, A Mocuta, A Ajmera, Tech Digest $\operatorname{IEDM}(2001 \mathrm{a}) \mathrm{p} 455$

18. J Koomen, Solid-State Electronics, 16801 (1973)

19. C G Sodini, T W Ekstedt, J L Moll, Solid-State Electronics, 25833 (1982)

20. J Robertson, J Vac Sci Technol B 181785 (2000)

21. H J Hubbard, D G Schlom, J Mater Res 112757 (1996);

22. $\quad$ D G Schlom, J H Haeni, MRS Bull 27198 (2002)

23. M Copel, M Gribelyuk, E Gusev, App Phys Lett 76436 (2000)

24. M Gutowski, J E Jaffe, C L Liu, M Stoker, R I Hegde, R S Rai, P J Tobin, App Phys Lett 801897 (2002)

25. Y Y Wu, M Y Yang, A Chin, W J Chen, C M Kwei, IEEE ED Lett 21341 (2000)

26. H Iwai, et al, Tech Digest IEDM (IEEE, 2002); M K J Okamoto, H Iwai, proc ESSDERC (2007) p199

27. J Kwo et al, App Phys Lett 77130 (2000)

28. A Fissel, H J Osten, E Bugiel, J Vac Sci Technol B 211765 (2003)

29. G D Wilk, R Wallace, J M Anthony, J App Phys 87484 (2000)

30. J-F Damlencourt, et al, Solid-State Elec 47, 1613 (2003).

31. G. Bersuker, et al, J. Appl. Phys. 100, 094108 (2006).

32. M P Agustin, G Bersuker, B foran, L A Boatner, S Stemmer, J App Phys 100, 024103 (2006)

33. A Deshpande, R Inman, G Jurisch, C.G.Takoudis J App Phys 99, 094102 (2006)

34. C. Maunoury, et al, J App Phys 101, 034112 (2007)

35. M T Ho, R T Brewer, L S Wiehlunski, Y J Chabal, N Moumen, M Boleslawski, App Phys Lett 87 $133103(2005)$

36. P E Batson, Microsc Microanal 11(Suppl 2) 2164 (2005). 
37. D. Schmeisser, F Zheng, F J Himpsel, H J Engelmann, E. Zschech, MRS Symp Proc, 917, E10-02 (2006)

38. P-G Mani-Gonzalez, M-O Vazquez-Lepe, F Espinosa-Magaña, A Herrera-Gomez, J. Vac. Sci. Technol A 31, 010601 (2013)

39. J Robertson, C W Chen, App Phys Lett 74 (1999) 1168

40. X Zhang, A Demkov, L Hao, X Hu, Y Wei, J Kulik, Phys Rev B 68125323 (2003)

41. E P Gusev, E Cartier, D A Buchanan, M Gribelyuk, M Copel, Microelec Eng 59341 (2001b)

42. S Guha, E Cartier, N A Bojarczuk, J Bruley, L Gignac, J Karasinski, App Phys Lett 90512 (2001)

43. S Guha, E P Gusev, H Okorn-Schmidt, L A Ragnarsson, N A Bojarczuk, App Phys Lett 812956 (2002)

44. W Tsai, et al (IMEC) Tech Digest IEDM (2003) paper 13.2

45. Y C Yeo, T J King, C Hu, App Phys Lett 812091 (2002)

46. M R Visokay, J J Chambers, A L P Rotondaro, A Shanware, L Colombo, App Phys Lett 803183 (2002)

47. R M Wallace, R A Stoltz, G D Wilk, US Pat. Nos. 6013553; 6020243 (2000); US Pat. Nos. 6291866; 6291866 (2001)

48. $\quad$ B H Lee, L Kang, R Nieh, W J Qi, J C Lee, App Phys Lett 761926 (2000)

49. H Kim, P C McIntyre, C O Chui, K C Saraswat, S. Stemmer, J Appl Phys 963467 (2004)

50. J Morais et al, App Phys Lett 812995 (2002); 794192 (2001)

51. M A Quevedo-Lopez, et al, App Phys Lett 811074 (2002);

52. $\quad$ M A Quevedo-Lopez, et al, App Phys Lett 824669 (2003)

53. $\quad$ R M C de Almeida, I J R Baumvol, Surf Sci Rep 491 (2003)

54. B W Busch, O Pluchery, Y J Chabal, D A Muller, R Opila, D A Muller, J R Kwo, E Garfunkel, Mat Res Soc Bull (March 2002) p206

55. S Ferrari, G Scarel, J App Phys 96144 (2004)

56. F J Himpsel, F R McFeely, A Taleb-Ibrahami, J A Yarnoff, G Hollinger, Phys Rev B 386084 (1988);

57. J W Keister et al, J Vac Sci Technol B 171250 (1999)

58. E H Poindexter, P J Caplan, Prog Surf Sci 14, 201 (1983)

59. R E Mikawa, P M Lenahan, J App Phys 59, 2054 (1986)

60. A Stesmans, Appl Phys Lett 68, 2723 (1996a); ibid. 68, 2076 (1996b)

61. A Stesmans, V V Afanasev, Phys Rev B57 10030 (1998)

62. C Hobbs et al, VLSI Symp (2003) p9

63. D C Gilmer et al, Microelectron Eng 69138 (2003)

64. C Hobbs et al, IEEE Trans ED 51971 (2004); 51978 (2004)

65. R Chau, J Brask, S Datta, G Dewey, M Doczy, B Doyle, J Kavalieros, M Radosavljevic, Microelectronic Eng 801 (2005)

66. K Xiong, P W Peacock, J Robertson, App Phys Lett 86012904 (2005)

67. M M Frank, Proc ESSDERC (2011)

68. J E Mahan, Physical Vapor Deposition of Thin Films, Wiley-Interscience (2000).

69. J L Vossen, W Kern, Thin Film Processes, Academic Press (1978); (1991)

70. J P Lehan, T Mao, B G Bovard, H A Macleod, Thin Sol Films 203, 227 (1991)

71. H Harris, K Choi, N Mehta, A Chandolu, N Biswas, G Kipshidze, S Nikishin, S Gangopadhyay, H Temkin, App Phys Lett 811065 (2002)

72. K Choi, H Temkin, H Harris, S Gangopadhyay, L Xie, M White, App Phys Lett 85, 215 (2004)

73. M Ritala, in 'High K gate dielectrics' ed M Houssa, (Inst Physics, Bristol, UK 2004)

74. M Ritala, K Kukli, A Rahtu, P I Raisanen, M Leskela, Science 288319 (2000)

75. S M George, Chem Rev 110111 (2010)

76. A C Jones, P R Chalker, J Phys D 36 R80 (2003)

77. M L Green, M Y Ho, B Busch, G D Wilk, T Sorsch, T Conard, B Brijs, W Vandervorst, P I Raisanen, D A Muller, M Bude, J Grazul, J App Phys 927168 (2002) 
78. L A Ragnarrson, N A Bojarczuk, J Karaninski, S Guha, IEEE ED Let 24689 (2003)

79. M M Frank, Y J Chabal, G D Wilk, App Phys Lett 824758 (2003)

80. M M Frank, Y J Chabal, M L Green, A Delabie, B Brijs, G D Wilk, M Y Ho, I J R Baumvol, App Phys Lett 83740 (2003)

81. D M Hausmann, E Kim, J Becker, R G Gordon, Chem Mat 144350 (2002)

82. D M Hausmann, R G Gordon, J Crystal Growth, 249251 (2003)

83. J S Becker, E Kim, R G Gordon, Chem Mats 163497 (2004); B S Lim, A Rahtu, R G Gordon, Nature Mats 2749 (2003)

84. T Q Ngo, A Posadas, M D McDaniel, D A Ferrer, J Bruley, C Breslin, A A DEmkov, J G Ekerdt, J Crystal Growth 363150 (2013); X Wang, L Dong, J Zhang, Y Liu, P D Ye, R G Gordon, Nanoletts 13 594 (2013)

85. G B Rayner, D Kang, G Lucovsky, J Vac Sci Technol B 211783 (2003)

86. J P Maria, D Wicaksana, A I Kingon, B Busch, H Schulte, E Garfunkel, T Gustafsson, J App Phys 903476 (2001)

87. S Stemmer, Y Li, B Foran, P S Lysaght, S K Streiffer, P Fuoss, S Seifert, App Phys Lett 833141 (2003)

88. $\quad$ H Kim, P C McIntyre, J App Phys 925094 (2002)

89. S Stemmer, Z Chen, C G Levi, P S Lysaght, B Foran, J A Gisby, J R Taylor, Jpn J App Phys 42 $3593(2003)$

90. C Zhao, O Richard, E Young, H Bender, G Roebben, S Haukka, S DeGendt, M Houssa, R Carter, W Tsai, O Van der Biest, M Heyns, J Non-Cryst Solids 303144 (2002)

91. S Stemmer, J Vac Sci Technol B 22791 (2004)

92. V Naraynan, S Guha, M Copel, N A Bojarczuk, P L Flaitz, M Bribelyuk, App Phys Lett 814183 (2002)

93. T Ando, et al, Tech Digest IEDM (2009); T Ando, et al, Tech Digest IEDM (2011);

94. T Ando, Materials 5478 (2012)

95. T Ando, M Copel, J Bruley, M M Frank, H Watanabe, V Narayanan, App Phys Lett 96132904 (2010)

96. M M Frank, E A Cartier, T Ando, S W Bedell, J Bruley, Y Zhu, V Narayanan, ECS Solid Letters 2 N8 (2013)

97. T Ando, B Kannan,. U Kwon, W L Lai, B P Linder, E A Cartier, R Haight, M Copel, JBruly, S A Krishnan, V Narayanan, Tech Digest VLSI Technol (2014) p6.1.

98. M.Copel, E Cartier, F M Ross, App Phys Lett 781607 (2001)

99. M Copel, E Cartier, V Narayanan, M C Reuter, S Guha, N Bojarczuk, App Phys Lett 814227 (2002)

100. H S Kim, P C McIntyre, C O Cui, K C Saraswat, S Stemmer, J App Phys 963467 (2004)

101. H Watanabe, M Saitoh, N Ikarashi, T Tatsumi, App Phys Lett 85449 (2004)

102. H Shimizu, K Kita, K Kyuno, A Toriumi, Jap J App Phys 446131 (2005)

103. P W Peacock, J Robertson, J App Phys 924712 (2002)

104. J H Choi, Y Mao, J P Chang, Mat Sci Eng R 7297 (2011)

105. R. H. French, J Am Ceram Soc 73477 (1990)

106. P Lamparter, R Kniep, Physica B 234405 (1997)

107. H Momida, T Hamada, Y Takagi, T Yamamoto, T Uda, T Ohno, Phys Rev B 73054108 (2006)

108. V V Afanaev, A Stesmans, J App Phys 102081301 (2007)

109. D Liu, J Robertson, App Phys Lett 94042904 (2009)

110. R H French, S J Glass, F S Ohuchi, Y N Xu, W Y Ching, Phys Rev B 495133 (1994)

111. J E Medvedeva, A J Freeman, C B Geller, D M Rishel, Phys Rev B 76235115 (2007)

112. H S Lee, S J Clark, J Robertson, Phys Rev B 86075209 (2012)

113. G Lucovsky, Y Zhang, J L Whitten, D G Schlom, J L Freeouf, Microelec Eng 72288 (2004)

114. G M Rignanese, X Gonze, A Pasquarello, Phys Rev B 63104305 (2001)

115. K Xiong, Y Du, K Tse, J Robertson, J App Phys 101024101 (2007) 
116. P Broqvist, A Pasquarello, App Phys Lett 90082907 (2007)

117. J Morais, L Miotti, K P Bastos, S R Teixeira, I J R Baumvol, A L P Rotondaro, J J Chambers, M R Visokay, L Colombo, M C MartinsAlves, App Phys Lett 86212906 (2005)

118. C A Pignedoli, A Curioni, W Andreoni, Phys Rev Lett 98037602 (2007)

119. H Kato, T Nango, T Miyagawa, T Katagiri, K S Seol, Y Ohki, J App Phys 921106 (2002)

120. K Xiong, J Robertson, S J Clark, App Phys Lett 89022907 (2006)

121. S G Lim, S Kriventsov, T N Jackson, J H Haeni, D G Schlom, A M Balbashov, R Uecker, P Reiche, J L Freeouf, G Lucovsky, J App Phys 914500 (2002)

122. D G Schlom, S Datta S Guha, MRS Bull (Nov 2008)

123. M Sousa, C Rossel, C Marchiori, H Siegwart, D Caimi, J P Locquet, D J Webb, R Germann, J Fompeyrine, K Babich, J W Seo, C Diker, J App Phys 102104103 (2007)

124. K Xiong, J Robertson, M C Gibson, S J Clark, App Phys Lett 87183505 (2005)

125. J Robertson, K Xiong, S J Clark, Phys Stat Solidi B 2432054 (2006)

126. A Alkauskas, P Broqvist, F Devynck, A Pasquarello, Phys Rev Lett 101106802 (2008)

127. P Broqvist, A Pasquarello, App Phys Lett 89082908 (2006)

128. B Kralik, E K Chang, S G Louie, Phys Rev B 577027 (1998)

129. J Drabowski, V Zavodinsky, A Fleszar, Microelect Reliab 411093 (2001)

130. X Zhao, D Vanderbilt, Phys Rev B 65233106 (2002)

131. G M Rignanese, X Gionze, G Jun, K J Cho, A Pasquarello, Phys Rev B 69184301 (2004)

132. P Delugas, V Fiorentini, A Filippetti, Phys Rev B 71134302 (2005)

133. J Robertson, J App Phys 104 123111(2008)

134. R D Shannon, J App Phys 73348 (1993)

135. R Hegde, et al, Tech Digest IEDM (2005) p59

136. R I Hegde, D H Triyoso, S B Samavedam, B E White, J App Phys 101074113 (2007)

137. D H Triyoso, R I Hegde, J K Schaeffer, J Vac Sci Technol B 25845 (2007)

138. K Tomida, K Kita, A Toriumi, App Phys Lett 89142902 (2006)

139. D Tsoutsou, G Apostopoulos, S F Galata, P Tsipas, A Sotiropoulos, G Mavrou, Y Panayiotatos, A Dimoulas, A Lagoyannis, A G Karydas, V Kantarelou, S Harrissopoulos, J App Phys 106024107 (2009)

140. P Li, I W Chen, J E Penner-Hahn, J Am Ceram Soc 77118 (1991)

141. J P Goff, W Hayes, S Hull, M T Hutchings, K N Clausen, Phys Rev B 5914202 (1999)

142. G Stapper, M Bernasconi, N Nicoloso, M Parrinello, Phys Rev B 59797 (1999)

143. D Fischer, A Kersch, App Phys Lett 92012908 (2008)

144. C K Lee, E Cho, H S Lee, C S Hwang, S Han, Phys Rev B 78012102 (2008)

145. L F Edge, D G Schlom, S A Chambers, E Cicerrella, J L Freeouf, B Hollander, J Schubert, App Phys Lett 84726 (2004)

146. L F Edge, D G Schlom, P Sivasubramani, R M Wallace, B Hollander, J Schubert, App Phys Lett 88 112907 (2006)

147. J M J Lopes, M Roekerath, T Heeg, E Rije, J Schubert, S Mantl, V V Afanasev, S Shamuilia, A Stesmans, Y Jia, D G Schlom, App Phys Lett 89222902 (2006)

148. J M J Lopes, M Roekerath, T Heeg, U Littmark, J Schubert, S Mantl, Y Jia, D G Schlom, Microelec Eng 841890 (2007)

149. J Schubert, O Trithaveesak, W Zander, D G Schlom, App Phys A 90577 (2008)

150. M Wagner, et al, App Phys Lett 88172901 (2006)

151. R Thomas, et al, App Phys Lett 89232902 (2006)

152. A Laha, H J Osten, A Fissel, App Phys Lett 90113508 (2007)

153. E D Ozban, J Lopes, M Roeckerath, D G Schlom, J Schubert, S Mantl, App Phys Lett 93052902 (2008)

154. K Xiong, J Robertson, App Phys Lett 95022903 (2009)

155. S A Shevlin, A Curioni, W Andreoni, Phys Rev Lett 94146401 (2005)

156. E Bonera, G Scarel, M Fanciulli, P Delugas, V Fiorentini, Phys Rev Lett 94027602 (2005) 
157. A Baldereschi, A Baroni, R Resta, Phys Rev Lett 61734 (1988)

158. C G van de Walle, Phys Rev B 391871 (1989)

159. R T Tung, Phys Rev Lett 846078 (2000); R T Tung, Phys Rev B 64205310 (2001)

160. W Mönch, Phys Rev Lett 581260 (1987)

161. W Mönch, Surface Sci 300928 (1994)

162. J Tersoff, Phys Rev Lett 52465 (1984)

163. A W Cowley, S M Sze, J App Phys 363212 (1965)

164. J Robertson, Tech Digest IEDM (2009); App Phys Lett 94152104 (2009)

165. J Robertson, J Vac Sci Technol A 31050821 (2013)

166. S A Chambers, Y Liang, Z Yu, R Dropad, J Ramdani, K Eisenbeiser, App Phys Lett 771662 (2000)

167. R Ludeke, M T Cuberes, E Cartier, App Phys Lett 762886 (2000)

168. D J Maria, J App Phys 455454 (1974)

169. V V Afanasev, M Houssa, A Stesmans, M M Heyns, App Phys Lett 783073 (2001)

170. V V Afanasev, M Houssa, A Stesmans, M M Heyns, J App Phys 913079 (2002)

171. V V Afanasev, A Stesmans, F Chen, X Shi, S A Cambell, App Phys Lett 811053 (2002)

172. V. V. Afanesev, A. Stesmans, M. Passlack, and N. Medendrop, App Phys Lett 85597 (2004)

173. S Sayan, E Garfunkel, S Suzer, App Phys Lett 802135 (2002)

174. G B Rayner, D Kang, Y Zhang, G Lucovsky, J Vac Sci Technol B 201748 (2002)

175. Oshima, S Toyoda, T Okumura, J Okabayashi, H Kumigashira, App Phys Lett 832172 (2003)

176. O. Reynault, N T Barrett, D Samour, S Quiais-Marthon, Surface Sci 566526 (2004)

177. C C Fulton, R J Nemanich, J App Phys 962665 (2004)

178. T Hattori, T Yosihda, T Shiraishi, K Takahashi, H Nohira, S Joumori, K Nakajima, M Suzuki, K Kimura, I Kashiwagi, C Ohshima, S Ohmi, H Iwai, Microelect Eng 72283 (2004)

179. S Miyazaki, J Vac Sci Technol B 192212 (2001)

180. G Seguini, E Bonera, S Spiga, G Scarel, M Fanciulli, App Phys Lett 855316 (2004)

181. V. V. Afanasev, A Stesmans, D Droopad, App Phys Lett 89092103 (2006)

182. A Ohta, M Yamaoka, S Miyazaki, Microelectr Eng 72154 (2004)

183. J W Keister et al, J Vac Sci Technol B 171831 (1999)

184. J L Alay, M Hirose, J App Phys 811606 (1997);

185. J Robertson, B Falabretti, J App Phys 100014111 (2006)

186. V V Afanasev, M Badylevich, A Stesmans, App Phys Lett 93212104 (2008)

187. V Fiorentini, G Gulleri, Phys Rev Lett 89266101 (2002)

188. A Fissel, J Dabrowski, H J Osten, J App Phys 918986 (2002)

189. P W Peacock, J Robertson, Phys Rev Letts 92057601 (2004)

190. P W Peacock, K Xiong, K Tse, J Robertson, Phys Rev B 73075328 (2006)

191. C J Forst, C Ashman, K Schwarz, P E Blochl, Nature 42756 (2004)

192. S J Wang, C K Ong, App Phys Lett 802541 (2002)

193. Y F Dong, Y P Feng, S J Wang, A C H Huan, Phys Rev B 72045327 (2005)

194. Y F Dong, S J Wang, J W Chai, Y P Feng, A C H Huan, App Phys Lett 86132103 (2005)

195. Y F Dong, S J Wang, Y P Feng, A C H Huan, Phys Rev B 73045302 (2006);

196. R Puthenkovilakam, E A Carter, J P Chang, Phys Rev B 69155329 (2004)

197. J Garavin, L Fonseca, G Bersuker, A L Shluger, Microelectronic Eng 80412 (2005)

198. P Broqvist, A Pasquarello, Microelec Eng 842022 (2007);

199. J Robertson, Solid State Electronics 49283 (2005)

200. A S Foster, V B Sulimov, F L Gejo, A L Shluger, R N Nieminen, Phys Rev B 64224108 (2001)

201. A S Foster, F L Gejo, A L Shluger, R N Nieminen, Phys Rev B 65174117 (2002)

202. K Xiong, J Robertson, J App Phys 99044105 (2006)

203. K Torii, K Shirashi, S Miyazaki, K Yamabe, M Boero, T Chikyow, K Yamada, H Kitajima, T Arikado, Tech Digest IEDM (2004) p129

204. J Kang, E C Lee, K J Chang, Y G Jin, App Phys Lett 843894 (2004) 
205. W L Scopel, A J R da Silva, W Orellana, A Fazzio, App Phys Lett 841492 (2004)

206. Y. P. Feng, A T L Lim, and M F Li, App Phys Lett 87062105 (2005)

207. M Ikeda et al, Mat Res Soc Symp Proc 786 E5.4 (2004)

208. D M Ramo, A L Shluger, J L Gavartin, Phys Rev Lett 99155504 (2007)

209. J X Zheng, G Ceder, W K Chim, Phys Stat Solidi RRL 2227 (2008)

210. C Tang, R Ramprasad, PRB 81161201 (2010)

211. J L Gavartin, D M Ramo, A L Shluger, G Bersuker, App Phys Lett 89082908 (2006)

212. E A Choi, K J Chang, App Phys Lett 94122901 (2009)

213. J L Lyons, A Janotti, C G Van de Walle, Microelec Eng 881452 (2011)

214. J Robertson, S J Clark, Phys Rev B 83075205 (2011)

215. A Y Kang, P M Lenahan, J F Conley, App Phys Lett 833407 (2003)

216. G Bersuker, P Zeitzoff, G Brown, H R Huff, Mats Today 7 (Jan 2004) p26

217. S Zafar, A Callegari, E Gusev, M V Fischetti, J App Phys 939298 (2003)

218. A Kerber, E Cartier, L Pantisano, R Degraeve, T Kauerauf, Y Kim, G Groeseneken, H E Maes, U Schwalke, IEEE ED Lett 2487 (2003)

219. J. Mitard, C. Leroux, G. Ghibaudo, G. Reimbold, X. Garros, B. Guillaumot, and F. Boulanger, Microelectronic Eng 80362 (2005)

220. E A Cartier, B P Linder, V Narayanan, V K Paruchuri, Tech Digest IEDM (2006)

221. W He, T P Ma, App Phys Lett 835461 (2003)

222. E P Gusev, C P D’Emic, App Phys Lett 835223 (2003)

223. H Takeuchi, D Ha, T J King, J Vac Sci Technol A 221337 (2004)

224. N V Nguyen, A V Davydov, D Chandler-Horowitz, M MM Frank, App Phys Lett 87192903 (2005)

225. SA Walsh, L Fang, J K Schaffer, E Weisbrod, L J Brillson, App Phys Lett 90052901 (2007)

226. S Guha, Phys Rev Let 98196101 (2007)

227. S Guha, P Solomon, App Phys Lett 92012909 (2008)

228. C Ko, M Shandalov, P C McIntyre, S Ramanathan, App Phys Lett 97082102 (2010)

229. S Zafar, H Jagannath, L F Edge, D Gupta, App Phys Lett 98152903 (2011)

230. A Stesmans, V V Afanas'ev, Appl Phys Lett 80, 1957 (2002)

231. A Stesmans, V V Afanasev, App Phys Lett 824074 (2003)

232. R C Barklie, S Wright, J Vac Sci Technol B 27317 (2009)

233. R Gillen, S J Clark, J Robertson, App Phys Lett (2012)

234. M Houssa, V V Afanasev, A Stesmans, M M Heyns, App Phys Lett 771885 (2000)

235. H S Kim, P C McIntyre, K C Saraswat, App Phys Lett 82106 (2003)

236. N Umezawa, K Shiraishi, T Ohno, H Watanabe, T Chikyow, K Torii, K Yamabe, K Yamada, H Kitajima, T Arikado, App Phys Lett 86143507 (2005)

237. J L Gavartin, A L Shluger, A S Foster, G I Bersuker, J App Phys 97053704 (2005)

238. N Shang, P W Peacock, J Robertson, App Phys Lett 84106 (2004)

239. N Umezawa, K Shiraishi, App Phys Lett 91132904 (2007)

240. D Liu, J Robertson, App Phys Lett 94042904 (2009)

241. H H Tseng, et al, IEDM (2005)

242. P McIntyre, et al, IEDM (2005)

243. K Tse, J Robertson, App Phys Lett 89142914 (2006); Microelec Eng 84663 (2007)

244. H Li, Y Guo, J Robertson, App Phys Lett 104192904 (2014)

245. J R Weber, A Janotti, C G van de Walle, J App Phys 109033715 (2011); M Choi, A Janotti, C G Van de Walle, J App Phys 113044501 (2013)

246. C C Yeh, T P Ma, N Ramasawamy, N Rocklein, D Gealy, T Graettinger, K Min, App Phys Lett 91 113521 (2007)

247. M Specht, M Stadele, S Jakschik, U Schroder, App Phys Lett 843076 (2004)

248. R DeGraeve et al, Tech Digest IEDM (2008) p353

249. X F Zheng et al, Tech Digest (2009) p139 
250. K Xiong, J Robetson, S J Clark, App Phys Lett 89022907 (2006); K Xiong, J Robertson, App Phys Lett 95022903 (2009)

251. H B Michaelson, J App Phys 484729 (1977)

252. Y C Yeo, T J King, C Hu, J App Phys 927266 (2002)

253. M Koyama, Y Kamimuta, T Ino, A Kancko, S Inumiya, K Eguchi, M Takayanagi, A Nishiyama, Tech Digest IEDM (2004) p499

254. S Zafar, et al, Tech Digest VLSI symp (IEEE, 2005) p44

255. J K Schaeffer, C Capasso, L Fonseca, S Samavedam, D Gilmer, Y Liang, O Adetutu, C Hobbs, E Lckowski, R Gregory, Z X Jiang, K Moore, B Y Nguyen, D Roan, B E White, P J Tobin, Tech Digest IEDM (2004), p287

256. J K Schaeffer, L R C Fonseca, S B Samavedam, Y Liang, P J Tobin, B E White, App Phys Lett 85 $1826(2004)$

257. E Cartier, F R McFeely, V Narayanan, P Jamison, M Copel, G Shahidi, Tech Digest VLSI Symp (IEEE, 2005) p 15

258. P Majhi et al, presented at MRS (Spring 2005)

259. H C Wen, P Majhi, K Choi, C S Park, H N Alshareef, H R Harris, H Luan, H Miimi, H B Park, G Bersuker, P S Lysaght, D L Kwong, S C Song, B H Lee, R Jammy, Microelec Eng 852 (2008)

260. R Jha, J Gurganos, Y H Kim, R Choi, J Lee, V Misra, IEEE ED Lett 25420 (2004); H C Wen, et al, IEEE ED Letts 27598 (2006)

261. H H Tseng et al, Tech Digest IEDM (2004) p821

262. J K Schaeffer, C Capasso, R Gregory, D Gilmer, L R C Fonseca, M Raymond, C Happ, M Kottke, S B Samavedam, P J Tobin, B E White, J App Phys 101014503 (2007);

263. V Narayanan, V K Paruchuri, N A Bojarczuk, B P Linder, B Doris, Y H Kim, S Zafar, J Stathis, S Brown, J Arnold, M Copel, E Cartier, A Callegari, S Guha, G Shahidi, T C Chen, Tech Digest VLSI symp (IEEE, 2006) p178;

264. V Narayanan, K Maitra, B P Linder, V K Paruchuri, E P Gusev, M M Frank, M L Steen, D LaTulipe, J Arnold, R Carruthers, D L Lacey, E Cartier, IEEE ED Lett 27501 (2006);

265. K. Tse, J. Robertson, Phys. Rev. Lett. 99086805 (2007)

266. K Tse, D Liu, J Robertson, Phys Rev B 81035325 (2010)

267. P W Tasker, J Phys C 124977 (1979)

268. C Noguera, J Phys Cond Mat 12 R367 (2000)

269. K Shiraishi, K Yamada, K Torii, Y Akasaka, K Nakajima, M Konno, T Chikyow, H Kitajima, T Arikado, Jpn. J. Appl. Phys. 43 L1413 (2004) poly

270. Y Akasaka, G Nakamura, K Shiraishi, N Umezawa, K Yamabe, O Ogawa, K Nakamura, Jpn. J. Appl. Phys. 45 L1289 (2006)

271. J Robertson, O Sharia, A A Demkov, App Phys Lett 91132912 (2007)

272. E Cartier, M Hopstaken, M Copel, App Phys Lett 95042901 (2009)

273. S C Song, C S Park, J Price, R Choi, H H Tseng, B H Lee, R Jammy, IEDM (2007) 13.3

274. K Akiyama, W Wang, W Mizubayashi, M Ikeda, H Ota, T Nabatame, A Toriumi, Tech Digest VLSI (IEEE, 2008) p8.3

275. J Schaeffer, D C Gilmer, S Samevadam, M Raymond, A Haggag, S Kalpat, B Seimie, C Capasso, B E White, J App Phys 102074511 (2007)

276. G Bersuker, C S Park, H C Wen, K Choi, J Price, P Lysaght, H H Tseng, O Sharia, A Demkov, J T Ryan, P Lenahan, IEEE Trans ED 572047 (2010)

277. V Narayanan, V K Paruchuri, N A Bojarczuk, B P Linder, B Doris, Y H Kim, S Zafar, J Stathis, S Brown, J Arnold, M Copel, E Cartier, A Callegari, S Guha, G Shahidi, T C Chen, Tech Digest VLSI symp (IEEE, 2006) p178;

278. S Guha, V K Paruchuri, M Copel, V Narayanan, Y Y Wang, P E Batson, N A Bojarczuk, B Linder, B Doris, App Phys Lett 90092902 (2007) 
279. H N AlShareef, M Quevedo-Lopez, H C Wen, R Harris, P Kirsch, P Majhi, B H Lee, R Jammy, D J Lichtenwalner, J S Jur, A I Kingon, App Phys Lett 89232103 (2006); H N AlShareef, K Choi, H C Wen, H Luan, H Harris, Y Senzaki, P Majhi, B H Lee, R Jammy, App Phys Lett 88072108 (2006)

280. H N Alshareef, H F Luan, K Choi, H R Harris, H C Wen, M A Quevedo-Lopez, P Majhi, B H Lee, App Phys Lett 88112114 (2006)

281. H J Li, M I Gardner, IEEE ED Letts 26441 (2005)

282. X P Wang, M F Li, A Chin, C X Zhu, J Shao, W Lu, X C Shen, X F Yu, R Chi, C Shen, A C H Huan, J S Pan, A Y Du, P Lo, D S H Chan, D L Kwang, Solid State Electronics 50986 (2006)

283. Y Yamamoto, K Kita, K Kyuno, A Toriumi, Jpn J App Phys 467251 (2007)

284. K Iwamoto, Y Kamimuta, A Ogawa, Y Watanabe, S Migata, T Nabatame, A Toriumi, App Phys Lett 92132907 (2008)

285. P D Kirsch, P Sivasubramani, J Huang, C D Young, M A Quevedo-Lopez, H C Wen, H Alshareef, K Choi, C S Park, B H Lee, H H Tseng, App Phys Lett 92092901 (2008)

286. H Jagannathan, V Narayanan, S Brown, Electrochem Soc Trans 1619 (2008)

287. J K Schaeffer, et al, Microelec Eng 842196 (2007)

288. K Kita A Toriumi, App Phys Lett 94132903 (2009)

289. L Lin, J Robertson, App Phys Lett 95012906 (2009)

290. L Lin, J Robertson, J App Phys 109094502 (2011)

291. O. Sharia, A A Demkov, G Bersuker, B H Lee, Phys Rev B 75035306 (2008); 77085326 (2008)

292. B P Linder, V Narayanan, V K Paruchuri, E Cartier, S Kanakasabapathy, Tech Digest SSDM (Tsukuba, 2007) p16

293. S Takagi, A Toriumi, M Iwase, H Tango, IEEE Trans ED 412357 (1994)

294. L A Ragnarsson, S Guha, M Copel, E Cartier, N A Bojarczuk, J Karasinski, App Phys Lett 784169 (2001)

295. S Guha, N A Bojarczuk, V Narayanan, App Phys Lett 80766 (2002)

296. M Hiratani, S Saito, Y Shimamoto, K Torii, Jpn J App Phys 414521 (2002)

297. K Torii et al, Microelectron Eng 65447 (2003)

298. M V Fischetti, D A Neumayer, E A Cartier, J App Phys 904587 (2001)

299. S Saito, D Hisamoto, S Kimura, M Hiratani, Tech digest IEDM (2003) 33.3

300. W Zhu, J P Han, T P Ma, IEEE Trans-ED 5198 (2004)

301. L A Ragnarsson, L Pantisano, V Kaushik, S I Saito, Y Shimamoto, S DeGendt, M Heyns, Tech Digest IEDM (2003) p87

302. Z Ren, M V Fischetti, E P Gusev, E A Cartier, M Chudzik, Tech Digest IEDM (2003) paper 33.2

303. M Casse, L Thevenod, B Guillaumot, L Tosti, F Martin, J Mitard, O Weber, F Andrieu, T Ernst, G Reimbold, T Billon, M Mouis, F Boulanger, submitted to IEEE Trans ED (2005)

304. V Narayanan, K Maitra, B P Linder, V K Paruchuri, E P Gusev, M M Frank, M L Steen, D LaTulipe, J Arnold, R Carruthers, D L Lacey, E Cartier, IEEE ED Lett 27501 (2006);

305. L A Ragnarsson, et al, Microelec Eng (2011); Tech Digest IEDM (2010)

306. A Kerber, E A Cartier, IEEE Trans Device Mater Reliab 9147 (2009)

307. E A Cartier, et al, Tech Digest IEDM (2011)

308. B Kaczer et al, Microelec Eng 861894 (2010)

309. J Franco et al, Tech Digest IEDM (2010) p4.1

310. J Franco, B Kaczer, P J Roussel, J Mitard, M Cho, L Witters, T Grasser, G Groeseneken, IEEE Trans ED 60396 (2013)

311. T Ando, E A Cartier, J Bruly, K Choi, V K Narayanan, IEEE EDL 34729 (2013)

312. A Veloso, et al, Tech Digest VLSI (2011) p 34

313. S Hyun et al, Tech Digest VLSI (2011) p32

314. M Bosi, G Attolini, Prog. Crystal Growth and Charact. Mat. 56146 (2010).

315. R Pilarisetty, Nature 479324 (2011)

316. S Takagi, IEDM short course (2012)

317. P S Goley, M K Hudait, Materials 72310 (2014) 
318. Y. Kamata, Materials Today 11(1-2) 30 (2008)

319. C Claeys, J Mitard, G Hellings, C Eneman, B DeJaeger, L Witters, R Loo, A Delablie, A Sioncke, M Caymax, ECS Trans 5425 (2013)

320. E Simeon, J Mitard, G Hellings, Mat Sci Semiconductor Proc 15588 (2012)

321. Q Xie, S Deng, M Schaekers, D Lin, M Caymax, A Delablie, X P Qu, Y L Jiang, D Deduytsche, C Detavernier, Semicond Sci Technol 27074012 (2012)

322. C O Chui, et al Tech Digest IEDM (2002) p437

323. H L Shang, K L Lee, P Kowlowski, C D'Emic, B E Sikorski, M Ieong, H S P Wong, K Guarini, W E Haensch, IEEE ED Lett 25135 (2004)

324. A Dimoulas, et al, App Phys Lett 86032908 (2005)

325. K Kita, S Suzuki, H Nomura, T Takahashi, T Nishimura, A Toriumi, Jpn J App Phys 472349 (2008)

326. A Toriumi, T Tabata, C H Lee, T Nishimura, K Kita, K Nagashio, Microelec Eng 861571 (2009)

327. C H Lee, T Tabata, T Nishimura, K Nagashio, K Kita, A Toriumi, App Phys Expr 2071404 (2009)

328. D Kuzum, T Krishnamohan, A Nainani, Y Sun, P A Pianetta, H S-P Wong, K C Saraswat, IEEE IEDM Tech Digest (2009) 453

329. T Sasada, Y Nakakita, M Takenaka, S Takagi, J App Phys 106073716 (2009)

330. A Delabie, et al, App Phys Lett 91082904 (2007)

331. K Kita, S K Wang, M Yoshida, C H Lee, K Nagashio, T Nishimura, A Toriumi, IEDM Tech Digest (2009) p29.6

332. S Wang, K Kita, T Nishimura, K Nagashio, A Toriumi, Jpn J App Phys 50 04DA01 (2011)

333. C H Lee, T Nishimura, K Nagashio, K Kita, A Toriumi, IEEE Trans ED 581295 (2011) p209

334. C H Lee, C Lu, T Tabata, W F Zhang, T Nishimura, K Nagashio, A Toriumi, Tech Digest IEDM (2013) p 2.5

335. V V Afanasev, A Stesmans, App Phys Lett 842319 (2004)

336. V Afanasev, A Stsmans, A Delabie, F Bellenger, M Houssa, M Meuris, App Phys Lett 92022109 (2008)

337. A Ohta, S Miyazaki, et al, J Surf Sci Nanotech 4174 (2006)

338. P Boqvist, F Binder, A Pasquarello, App Phys Lett 94141911 (2009)

339. H Li, K Xiong, J Robertson, App Phys Lett 97242902 (2010)

340. A Dimoulas, App Phys Lett 89252110 (2006)

341. J R Weber, A Janotti, P Rinke, C G van de Walle, App Phys Lett 91142101 (2007)

342. E H Poindexter, G J Gerardi, M E Rueckel, P J Caplan, N M Johnson, D K Biegelsen, J App Phys 562844 (1984)

343. P Broqvist, A Alkauskas, A Pasquarello, Phys Rev B 78075203 (2008)

344. T Nishimura, K Kita, A Toriumi, App Phys Lett 91123123 (2007)

345. H Seo et al, Phys Rev B 89115318 (2014)

346. K L Brower, App Phys Lett 431111 (1983)

347. M Houssa, G Pourtois, M Caymax, M Meuris, M M Heyns, V Afanasev, A Stesmans, App Phys Lett 93161909 (2008)

348. A Stesmans, P Somers, V V Afanasev, Phys Rev B 79195301 (2009)

349. P Somers, A Stesmans, L Souriau, V V Afanasev, J App Phys 112074501 (2012)

350. S Baldovino, M Fanciuli, App Phys Lett 93242105 (2008)

351. S Baldovino, A Molle, M Fanciulli, App Phys Lett 96222110 (2010)

352. S Paleari, A Baldovino, A Molle, M Fanciulli, Phys Rev Lett 110206101 (2013)

353. A Stesmans, T N Hoang, V V Afanasev, J App Phys 116044501 (2014)

354. P Broqvist, J F Binder, A Pasquarello, Physica B 4072926 (2012)

355. H Li, J Robertson, Microelec Eng 109244 (2013); H Li, J Robertson, given at SISC, San Diego (2013)

356. R M Wallace, P C McIntyre, J Kim, Y Nishi, MRS Bulletin 34493 (2009)

357. H Kim, C O Chui, K C Saraswat, P C McIntyre App Phys Lett 832647 (2003) 
358. S Takagi, T Maeda, N Taoka, M Nishizawa, Y Morita, K Ikeda, Y Yamashita M Nishikawa, H Kumagai, R Nakane, S Sugahara, N Sugiyama, Microelectronic Eng 842314 (2007)

359. M Caymax, M Houssa, G Pourtois, F Bellenger, K Martens, A Delabie, S Van Elshocht, App Surface Sci 2546094 (2008)

360. M Milojevic, R Contreras-Guerrero, M Lopez-Lopez, J Kim, R M Wallace, App Phys Lett 95 212902 (2009)

361. R Zhang, N Taoka, P-C Huang, M Takenaka, S Takagi, IEEE IEDM Tech Digest (2011), 642 (a)

362. R Zhang, T Iwasaki, N Taoka, M Takenaka, S Takagi, Microelectronic Eng 88, 1533 (2011) (b)

363. R Zhang, T Iwasaki, N Taoka, M Takenaka, S Takagi, Tech Digest VLSI Symp (2011), 56 (c)

364. R Zhang, T Iwasaki, N Taoka, M Takenaka, S Takagi, J Electrochem Soc, G178 (2011) (d)

365. D Kuzum, J-H Park, T Krishnamohan, H S-P Wong, K C Saraswat, IEEE Trans Electron Dev 58 $1015(2011)$

366. H Kim, P C McIntyre, C O Chui, K C Saraswat, M H Cho, App Phys Lett 852902 (2004)

367. C O Chui, F Ito, K C Saraswat, IEEE Trans. Electron Dev 531501 (2006) (a)

368. C O Chui, H Kim, D Chi, P C McIntyre, K C Saraswat, IEEE Trans. Electron Dev 531509 (2006)

369. Y Oshima, M Shandalov, Y Sun, P Pianetta, P C McIntyre, App Phys Lett 92183102 (2009)

370. Q Xie, J Musschoot, M Schaekers, M Caymax, A Delabie, X P Qu, Y L Jiang, S V Berghe, J Liu, C Detavernier, App Phys Lett 97, 222902 (2010)

371. S Sioncke, H C Lin, L Nyns, G Brammertz, A Delabie, T Conard, A Franquet, J Rip, S DeGendt, M Mulle,r B Beckhoff, M Caymax, J App Phys 110084907 (2011)

372. A Delabie, S Sioncke, J Rip, S Van Elshocht, M Caymax, G Pourtois, K Pierloot, J Phys. Chem C 115, $17523(2011)$

373. C Fleischmann, K Schouteden, M Muller, P Honicke, B Beckhoff, S Sioncke, H G Boyen, M Meuris, C V Hasendoek, K Temst, A Vantomme, J Mat Chem C 14105 (2013)

374. J S Lee,T Kaufman-Osborn, W Melitz, S Lee, A Delabie, S Sioncke, M Caymax, G Pourtois, A C Kummel, J Chem Phys 135054705 (2011)

375. R Zhang, N Taoka, P C Huang, M Takenaka, S Takagi, Tech Digest IEDM (2011) p28.3

376. H Li, J Robertson, App Phys Lett 101052903 (2013)

377. R Zhang, P C Huang, J C Lin, M Takenaka, S Takagi, Tech Digest IEDM (2012) p16.1

378. R Zhang, J C Lin, X Yu, M Takenaka, S Takagi, Tech Digest VLSI (2013) p3.1

379. R Zhang, P C Huang, J C Lin, M Takenaka, S Takagi, App Phys Lett 102081603 (2013)

380. C H Lee, C Lu, T Tabata, K Nagashio, A Toriumi, Tech Digest VLSI (2013) p3.2

381. S Swaminathan, Y Oshima, M A Kelly, P C McIntyre, App Phys Lett 95, 032907 (2009)

382. J Han, R Zhang, T Osada, M Hata, M Takenada, S Takagi, App Phys Expr 6051302 (2013)

383. A Toriumi et al, Tech Digest IEDM (2009)

384. R Zhang et al, Tech Digest IEDM (2012)

385. R Zhang et al, Tech Digest IEDM (2013) 
Table 1. Static dielectric constant (K), experimental band gap and (consensus) conduction band offset on $\mathrm{Si}$ of the candidate gate dielectrics.

\begin{tabular}{|l|l|l|l|}
\hline & K & Gap (eV) & CB offset (eV) \\
\hline $\mathrm{Si}$ & & 1.1 & \\
\hline $\mathrm{SiO}_{2}$ & 3.9 & 9 & 3.2 \\
\hline $\mathrm{Si}_{3} \mathrm{~N}_{4}$ & 7 & 5.3 & 2.4 \\
\hline $\mathrm{Al}_{2} \mathrm{O}_{3}$ sapphire & 9 & 8.8 & 2.8 \\
\hline $\mathrm{Al}_{2} \mathrm{O}_{3} \mathrm{ALD}$ & 8 & 6.4 & 1.6 \\
\hline $\mathrm{Ta}_{2} \mathrm{O}_{5}$ & 22 & 4.4 & 0.35 \\
\hline $\mathrm{TiO}_{2}$ & 80 & 3.5 & 0 \\
\hline $\mathrm{SrTiO}_{3}$ & 2000 & 3.2 & 0 \\
\hline $\mathrm{ZrO}_{2}$ & 25 & 5.8 & 1.5 \\
\hline $\mathrm{HfO}_{2}$ & 25 & 5.8 & 1.4 \\
\hline $\mathrm{HfSiO}_{4}$ & 11 & 6.5 & 1.8 \\
\hline $\mathrm{La}_{2} \mathrm{O}_{3}$ & 30 & 6 & 2.3 \\
\hline $\mathrm{Y}_{2} \mathrm{O}_{3}$ & 15 & 6 & 2.3 \\
\hline $\mathrm{a}-\mathrm{LaAlO}_{3}$ & 30 & 5.6 & 1.8 \\
\hline $\mathrm{LaLuO}_{3}$ & 32 & 5.2 & 2.1 \\
\hline
\end{tabular}


Table 2. Precursor molecules for atomic layer deposition (ALD)

\begin{tabular}{|c|c|c|}
\hline type & Formula & comments \\
\hline chlorides & $\mathrm{MCl}_{4}$ & Non-volatile, corrosive byproduct \\
\hline alkyls & M-R & $\mathrm{Al}, \mathrm{Zn} \mathrm{OK}$, but few others \\
\hline alkoxides & M-OR & $\begin{array}{l}\text { Limited stability, better for MOCVD } \\
\text { than ALD }\end{array}$ \\
\hline Diketonates & & Low reactivity, high melting point \\
\hline Alkyl imide & $\mathrm{M}=\mathrm{N}-\mathrm{R}$ & Thermal stability \\
\hline Alkyl amides & $\mathrm{M}-\mathrm{NR}_{2}$ & good thermal stability \\
\hline Alkyl amido & $\mathrm{M}-\left(\mathrm{N}=\mathrm{RR}^{\prime}\right)_{\mathrm{n}}$ & good thermal stability \\
\hline Pentadienyl & & Low reactivity or low volatility \\
\hline nitrates & $\mathrm{M}\left(\mathrm{NO}_{3}\right)_{\mathrm{n}}$ & \\
\hline
\end{tabular}

Table 3. Comparison of the calculated conduction band offset (by LDA method) and experimental values for various gate oxides, by various authors. $*=$ ALD

\begin{tabular}{|l|l|l|l|}
\hline & Calculated (eV) & Experiment (eV) & Ref \\
\hline \multicolumn{1}{|c|}{$\mathrm{SiO}_{2}$} & & 3.1 & Alay (1999) \\
\hline $\mathrm{Ta}_{2} \mathrm{O}_{5}$ & 0.35 & 0.3 & Miyazaki (2001) \\
\hline $\mathrm{SrTiO}_{3}$ & 0.4 & 0 & Chambers (2000) \\
\hline $\mathrm{ZrO}_{2}$ & 1.6 & 1.4 & Miyazaki (2001) \\
& & 2.0 & Afanasev (2002) \\
& & 1.4 & Rayner (2002) \\
\hline $\mathrm{HfO}_{2}$ & 1.3 & 1.3 & Sayan (2002) \\
& & 2.0 & Afansev (2002) \\
\hline $\mathrm{Al}_{2} \mathrm{O}_{3}$ & 2.4 & 2.8 & Ludeke (2000) \\
& & $2.2 *$ & Afansev (2007) \\
\hline a-LaAlO & 1.0 & 1.8 & Edge (2004) \\
\hline $\mathrm{La}_{2} \mathrm{O}_{3}$ & 2.3 & 2.3 & Hattori (2004) \\
\hline $\mathrm{Y}_{2} \mathrm{O}_{3}$ & 2.3 & 1.6 & Miyazaki (2001) \\
\hline $\mathrm{LaLuO}_{3}$ & 2.2 & 2.1 & Lopes (2006) \\
\hline
\end{tabular}


Table 3 Physico-chemical properties of Ge compared to other semiconductors

\begin{tabular}{|l|l|l|l|}
\hline & $\mathrm{Si}$ & Ge & GaAs \\
\hline Band Gap $(\mathrm{eV})$ & 1.1 & 0.66 & 1.42 \\
\hline Electron mobility $\left(\mathrm{cm}^{2} / \mathrm{V} . \mathrm{s}\right)$ & 1500 & 3900 & 8500 \\
\hline Hole mobility $\left(\mathrm{cm}^{2} / \mathrm{V} . \mathrm{s}\right)$ & 450 & 1900 & 400 \\
\hline
\end{tabular}

Table 4. Properties of the Ge and Si oxide compared.

\begin{tabular}{|l|l|l|}
\hline & $\mathrm{SiO}_{2}$ & $\mathrm{GeO}_{2}$ \\
\hline$\Delta \mathrm{H}_{\mathrm{f}}(\mathrm{eV})$ & -4.80 & -3.27 \\
\hline Band gap (eV) & 9.0 & 6.0 \\
\hline Si-H bond energy (eV) & 3.3 & 3.1 \\
\hline CNL (of semiconductor) (eV) & 0.3 & 0 \\
\hline
\end{tabular}




\section{Fig captions}

1. The scaling of feature size, gate length, and oxide thickness according to the 2009 Semiconductor Roadmap

2. Leakage current vs voltage for various thickness of $\mathrm{SiO}_{2}$ layers, after [14].

3. TEM image of $\mathrm{SiO}_{2}$ based gate stack vs image of $\mathrm{HfO}_{2}$ gate stack.

4. The three contributions to the capacitance of the gate/electrode stack; channel, dielectric and gate depletion.

5. Schematic of replacement of $\mathrm{SiO}_{2}$ gate oxide and the poly-Si gate by high $\mathrm{K}$ gate oxide and metal gate, showing effect on gate capacitances.

6. Static dielectric constant vs band gap for candidate gate oxides [9].

7. Effect of reaction of $\mathrm{Si}$ with $\mathrm{ZrO}_{2}$ making $\mathrm{Zr}$ silicide.

8. $\quad$ Need for band offsets of over $1 \mathrm{~V}$.

9. Leakage current density vs. EOT for various high $\mathrm{K}$ oxides, for $\mathrm{HfO}_{2}$ (Gusev [17]), $\mathrm{ZrO}_{2}$ (Tsai [44]), $\mathrm{Al}_{2} \mathrm{O}_{3}$ (Gusev [17], Guha [42]) and $\mathrm{La}_{2} \mathrm{O}_{3}$ (Iwai [26]).

10. Comparison of the gate first and gate last (replacement gate, dummy gate) process.

11. Schematic of the cyclic process of Atomic Layer Deposition. Thanks to P C McIntyre.

12. Scanning electron microscope image of trench structure showing excellent coverage by ALD $\mathrm{HfO}_{2}$. Thanks to P C McIntyre.

13. Annealing of the infra-red spectrum of a $\mathrm{HfO}_{2}$ film grown by ALD with water as oxidant, showing the evolution of hydrogen [35].

14. Plan view TEM image of crystallisation in $\mathrm{HfO}_{2} / \mathrm{SiO}_{2}$ alloy system (a) $40 \% \mathrm{HfO}_{2}$, (b) $80 \% \mathrm{HfO}_{2}$ [89]. Thanks to S Stemmer.

15. (a) Phase diagram of $\mathrm{ZrO}_{2} / \mathrm{SiO}_{2}$ showing miscibility gap. After Kim and McIntyre [88].

(b) Phase diagram of $\mathrm{La}_{2} \mathrm{O}_{3} / \mathrm{SiO}_{2}$ with miscibility gap. After Maria[86].

16. HRTEM cross section showing interfacial layer of $\mathrm{SiO}_{2}$ below the $\mathrm{HfO}_{2}$ layer. Thanks to S Stemmer. (b) Schematic of need for MIPS to minimise oxygen ingress.

17. (a) Band structure and (b) Density of states of $\alpha-\mathrm{Al}_{2} \mathrm{O}_{3}$ (corundum). (c) Band structure of $\theta-\mathrm{Al}_{2} \mathrm{O}_{3}$

18. Local order in cubic, tetragonal and monoclinic $\mathrm{HfO}_{2}$ or $\mathrm{ZrO}_{2}$.

19. Band structure and density of states of cubic $\mathrm{HfO}_{2}$.

20. Band structure and density of states of cubic $\mathrm{ZrO}_{2}$.

21. Partial density of states of $\mathrm{La}_{2} \mathrm{O}_{3}$.

22. Partial density of states of crystalline $\mathrm{ZrSiO}_{4}$.

23. Bands and Density of states of cubic $\mathrm{LaAlO}_{3}$.

24. Density of states of cubic $\mathrm{LaLuO}_{3}$.

25. Free energy of formation of various oxides per $\mathrm{O}$ atom, vs. Parent metal work function. Equivalent to an Ellingham diagram.

26. Schematic of how charge transfer at semiconductor interface controls its band line up, a) no charge transfer, $b$ ) charge transfer

27. (a) Origin of metal induced gap states (MIGS) at metal-semiconductor interface, (b) presence of MIGS type states at a semiconductor-semiconductor heterojunction, only in energy range where band states exist.

28. Predicted barrier heights for a range of high $\mathrm{K}$ gate oxides, after Robertson [9].

29. Bonding at (100)Si:HfO $\mathrm{H}_{2}$ interfaces. O-terminated, non-polar and Hf-terminated interfaces. 
30. Bonding at Si-HfO2 interfaces. (a) unrelaxed O-terminated $\mathrm{O}_{4}$ interface, (b) relaxed $\mathrm{O}_{4}$ interface, (c) $\mathrm{O}_{3}$ interface, and (d) $\mathrm{O}_{2}$ interface.

31. Atomic bonding at relaxed metal-terminated interfaces (a) $\mathrm{Hf}_{6}$, (b) $\mathrm{Hf}_{10}$.

32. The relaxed structure of (a) neutral oxygen vacancy and (b) the neutral oxygen interstitial in $\mathrm{HfO}_{2}$

33. Calculated defect energy levels of the oxygen vacancy and $\mathrm{O}$ interstitial in $\mathrm{HfO}_{2}$ in various charge states.

34. Defect formation energy vs Fermi energy of $\mathrm{O}$ vacancy and $\mathrm{O}$ interstitial in cubic $\mathrm{HfO} 2$, in O-rich and O-poor condition. The stable charge state at any Fermi energy corresponds to the lowest energy.

35. Electron trapping in $\mathrm{HfO}_{2}$ gate oxide layer. The hysteresis between the up and down ramps shows the presence of sizable trapping. The identical curves for up and down show that no new defects are created.

36. Calculated defect wavefunction of $\mathrm{O}$ vacancy in $\mathrm{HfO}_{2}$, (a) in GGA and (b) SX, showing lowering of symmetry in SX result.

37. Passivatioon of $\mathrm{O}$ vacancy in $\mathrm{HfO}_{2}$ by introduction of two nitrogens. Atomic strcuture, schematic energy levels, and partial density of states. Note absence of deep gap states.

38. Passivatioon of $\mathrm{O}$ vacancy in $\mathrm{HfO}_{2}$ by introduction of two La atoms. Atomic strcuture, and partial density of states. Note absence of deep gap states.

39. Calculated defect formation energy vs Fermi energy of oxygen vacancy in $\theta-\mathrm{Al}_{2} \mathrm{O}_{3}$.

40. Calculated defect energy levels of oxygen vacancy in $\mathrm{La}_{2} \mathrm{O}_{3}$ and $\mathrm{LaAlO}_{3}$ in various charge states.

41. Prefered values of gate work functions for simple CMOS devices.

42. Schematic definition of effective work function (EWF). Energies referred to Si band edges, and thereby to the vacuum level.

43. (a) Effective work functions of metals on $\mathrm{HfO}_{2}$, from internal photoemission data (Afanasev) and from CV data cited by Yeo [252]. (b) effective work functions of 3 refractory metals on $\mathrm{HfO} 2 \mathrm{vs}$ on $\mathrm{SiO}_{2}$, by barrier height method, from Zafar [254], effective work functions of metals of $\mathrm{HfO} 2$ vs metal vacuum work function, from data of Koyama [253].

44. Effective work functions of metals on $\mathrm{HfO}_{2}$ against metal index, from Schaeffer [255].

45. Flat band voltage vs annealing temperature for $\mathrm{Re}-\mathrm{HfO}_{2}$ stacks cyclically annealed in oxygen and forming gas, from Cartier [257].

46. (a) Effective work functions of refractory metals on $\mathrm{HfO}_{2}, \mathrm{HfSiO}_{\mathrm{x}}$ and $\mathrm{SiO}_{2}$ from $\mathrm{Wen}$ [259]. (b) Data replotted as EWF on $\mathrm{HfO}_{2}$ vs. EWF on $\mathrm{SiO}_{2}$. This gives a slope $\mathrm{S} \sim 0.82$

47. O-terminated, non-polar, and metal-terminated interfaces of $\mathrm{FCC}$ metal on $\mathrm{HfO}_{2}$.

48. Calculated valence band offsets (VBO) of metals on $\mathrm{HfO}_{2}$ interfaces, for different interface terminations. Note $\mathrm{S}$ factor of 0.9 , and the large shift between the non-polar (110) interface and the polar (100) interface. EWF is calculated from the VBO.

49. (a) Schematic of oxygen vacancy creation and charge transfer from vacancy to gate electrode for a metal- $\mathrm{HfO}_{2}$-silicon stack. (b) resulting band bending due to positive vacancies.

50. Formation energy of $\mathrm{O}$ vacancies in $\mathrm{HfO} 2$ in contact with large excess of $\mathrm{Si}$, at the $\mathrm{Si} / \mathrm{SiO} 2$ equilibrium chemical potential. Note the very low formation energy of the $\mathrm{O}$ vacancy. 
51. Overall reaction, forming charge $\mathrm{O}$ vacancy, electrons going to the gate, and oxygen atom reacting with the $\mathrm{Si}$ to form more $\mathrm{SiO} 2$.

52. EWF of p-type metals on $\mathrm{HfO}_{2}$ vs EOT, showing the $\mathrm{V}_{\mathrm{T}}$ roll-off effect at low EOT values, after Lee et al [8].

53. Schematic of oxide capping layer on $\mathrm{HfO} 2$ gate oxide, in gate first approach.

54. Cross sectional TEM image, and lateral EELS scan for oxide composition across gate stack having a La capping layer. Note that La has moved to the $\mathrm{HfO} 2 / \mathrm{SiO} 2$ interlayer (IL) from its initial position at the TiN/HfO2 interface [276].

55. CV plots of different layer structures to find the active interface in capping layer system. Note that the Vt shift depends on nature of bottom layer, not the top layer.

56. Configurations of metals of different valence substitiing for $\mathrm{Hf}$ sites at a $\mathrm{SiO}_{2}: \mathrm{HfO}_{2}$ interface, in dipole layer scheme.

57. (a) Calculated valence band offset (VBO) vs experimental flat band voltage shift Vfb, for various metal oxides, for configurations of fig 56. (b) Calculated VBOs versus metal valence, showing anomalous case of $\mathrm{Al}$, (c) calculated VBOs vs metal work function, showing consistency with observed results.

58. Dipole mechanism from change of screening across $\mathrm{SiO} 2 / \mathrm{HfO} 2$ interface.

59. Schematic carrier mobility vs vertical (gate) field in FETs in the universal mobility model, showing the mechanisms which limit the mobility, and their temperature dependences.

60. (a), (b) electron and hole mobilities of FETS on $3 \mathrm{~nm} \mathrm{HfO} 2$ gate oxide on $1 \mathrm{~nm} \mathrm{SiO}_{2}$ with TiN gate electrodes, showing the stronger degradation of electron mobility [303].

61. Schematic of remote charge scattering, and remote phonon scattering, including the interfacial layer, which partially screens their effect.

62. Variation of additional mobility with thickness of the inter-layer, at different temperatures, for scaled oxides, after Ragnarsson [305].

63. Additional mobility vs gate field, at low fields and different temperatures, after Casse [303].

64. Mobility vs EOT for NMOS, using data from Nayaranan [264,10], Lee [12], Ragnarsson [303] and Ando [94].

65. Carrier injection into O vacancy levesl causing PBTI in nFET, and hole injection into $\mathrm{SiO} 2$ hole traps causing NBTI in pFETs, after Cartier [307].

66. PBTI and NBTI in HfO2 gate oxide compared to reference SiON system [307].

67. Normalised variation of PBTI and NBTI with changes in EOT for $\mathrm{HfO} 2$ gate oxide system, including scavenged oxide [307].

68. Maximum applied gate voltage to give $30 \mathrm{mV} \mathrm{Vt}$ shift after 10 year lifetime, for PBTI and NBTI, vs EOT, after Ragnrsson [305]

69. Schematic of reducing the oxide inter-layer by scavenging, for three different scavenger metal locations, after [94].

70. Cross sectional TEM showing reduction of $\mathrm{SiO}_{2}$ interlayer after scavenging.

71. EOT reduction by scavenging, vs free energy of oxide formation per $\mathrm{O}$ atom. Electropositive metals are aggressive scavengers. Optimum scavengers are less aggressive, but more electropositive than $\mathrm{Si}$, such as $\mathrm{Ti}$.

72. Temperature dependence of scavenging reaction, showing onset at the $\mathrm{O}$ diffusion temperature of 500C [94].

73. Electron mobility vs EOT for scavenged gate stack, at $1 \mathrm{MV} / \mathrm{cm}$ vertical field. 
74. Schematic of $\mathrm{V}_{\mathrm{T}}$ roll-off vs. EOT dependence for scavenged oxide gate last stacks, from [312], showing roll-off occurring at a smaller EOT value than for gate first stacks.

75. T inversion vs ITRS node, showing small EOTs acheieved by use of $\mathrm{HfO}_{2}$.

76. Flat band voltage of TiN gate electrodes for FinFET, TiN for nFET and TiON for pFET.

77. Cross sectional TEM image of (100) and (111) $\mathrm{Ge} / \mathrm{GeO}_{2}$ interfaces, showing their abruptness and flatness (Sasada [326])

78. Schematic of the $\mathrm{GeO}$ evolution mechanism, $\mathrm{O}$ vacancies are created at $\mathrm{Ge} / \mathrm{GeO}_{2}$ interface, causing traps, they diffuse across the oxide, and then react with the outside $\mathrm{GeO}_{2}$ layer to form $\mathrm{GeO}$ molecules [329].

79. Calculated partial DOS of $\mathrm{Ge} / \mathrm{GeO}_{2}$ interface, giving valence band offset of $4.1 \mathrm{eV}$. (b) band alignemtn at $\mathrm{Ge} / \mathrm{GeO}_{2}$ interface, showing small conduction band offset.

80. Temperature dependence of stress and $\mathrm{Pb}$ density vs anneal temperature, after Houssa [345].

81. Magnetic field dependence of the EDMR sugnal of $G e P_{b}$ signal [350].

82. Valence alternation pair defect formed by re-arrangement of a relaxed $\mathrm{O}$ vacancy in $\mathrm{GeO}_{2}$, consisting of a 3-fold $\mathrm{O}$ site and a 3-fold Ge site. 3-fold sote gives a state near Ev of $\mathrm{Ge}, 3$-fold $\mathrm{O}$ site gives a state near $\mathrm{Ec}$ of Ge.

83. In-situ Ge $2 p$ XPS spectra of ALD half cycles for (a) $\mathrm{Ge}(100)$ etched with deionized water, (b) clean $\mathrm{Ge}(100)$ surface, and (c) GeON surface. The $2 p$ photoelectron line is chosen to enhance surface sensitivity due to the low photoelectron kinetic energy $(\sim 270$ eV). After Milojevic 2009.

84. (a) The deconvoluted Ge $3 d$ XPS spectra after plasma oxidation of an $\mathrm{ALD} \mathrm{Al}_{2} \mathrm{O}_{3} / \mathrm{Ge}$ stack showing the dominance of the $\mathrm{Ge}^{3+}$ oxidation state. (b) The interface state density dependence on $\mathrm{GeO}_{\mathrm{x}}$ thickness and oxidation state.

85. Use of $\mathrm{Al}_{2} \mathrm{O}_{3}$ as diffusion barrier on $\mathrm{Ge}$ for improve $\mathrm{Ge} / \mathrm{GeO}_{2}$ gate stacks. Oxidation of underlying Ge by ECR plasma is accomplished through the $\mathrm{Al}_{2} \mathrm{O}_{3}$ layer.

86. Electron and hole mobilties vs EOT for various FETs [320,356,331,378-380] 


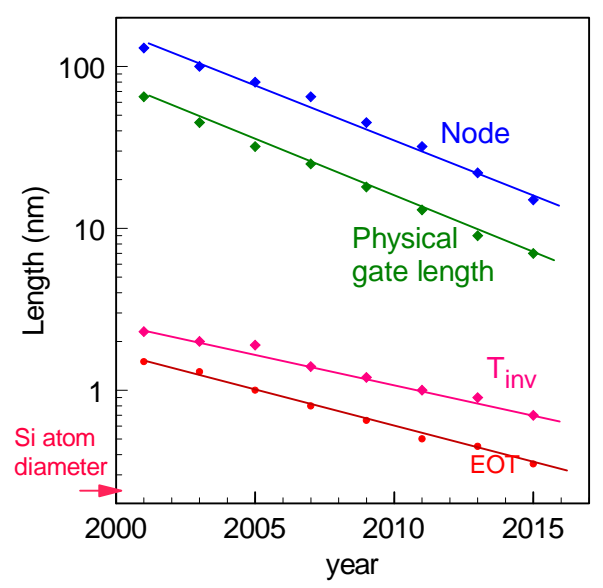

Fig. 1

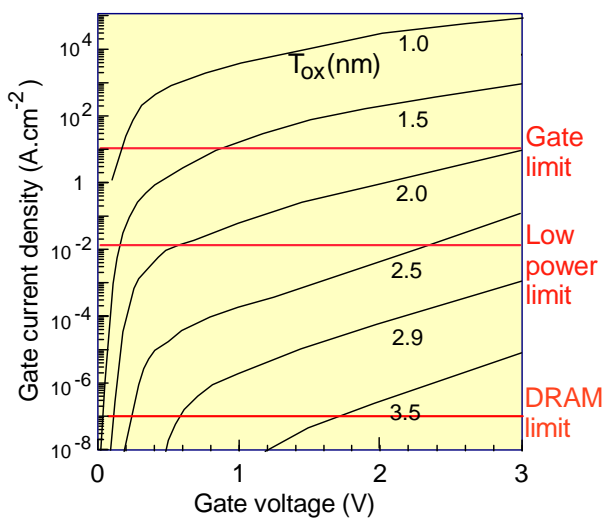

Fig 2
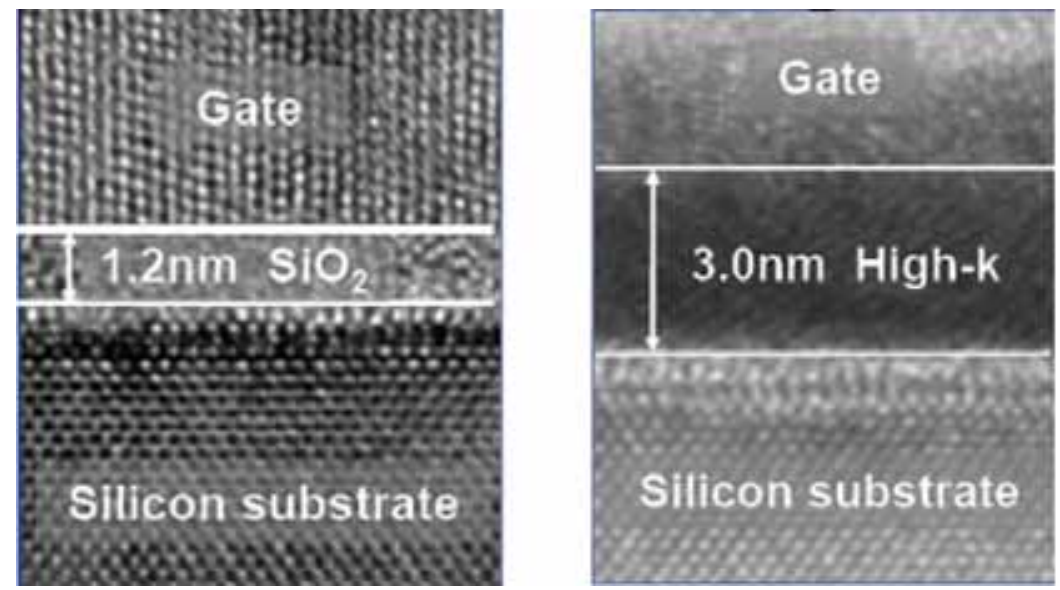

Fig 3 


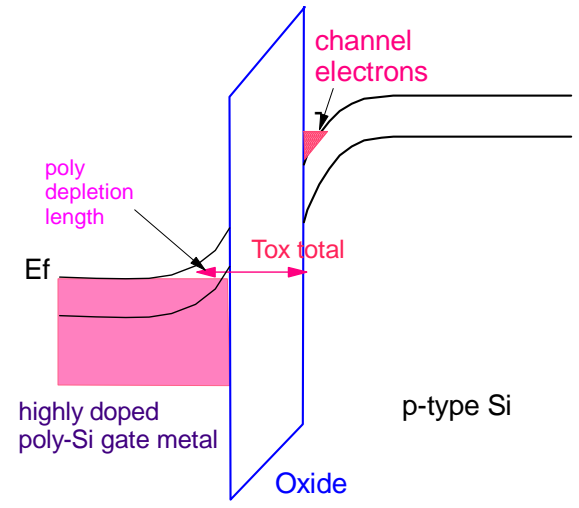

Fig 4

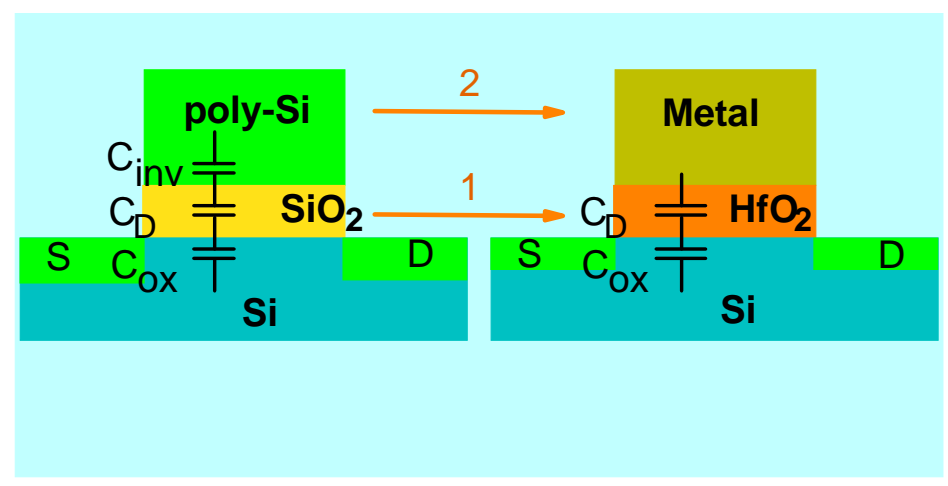

Fig. 5

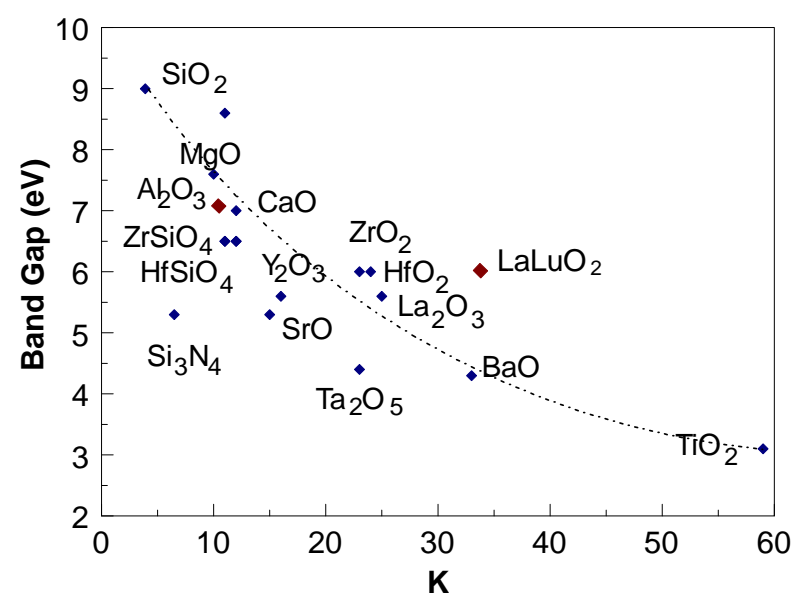

Fig 6 


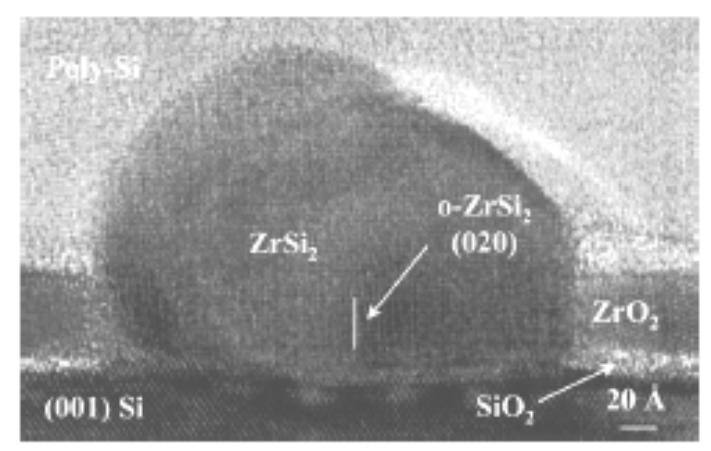

Fig 7

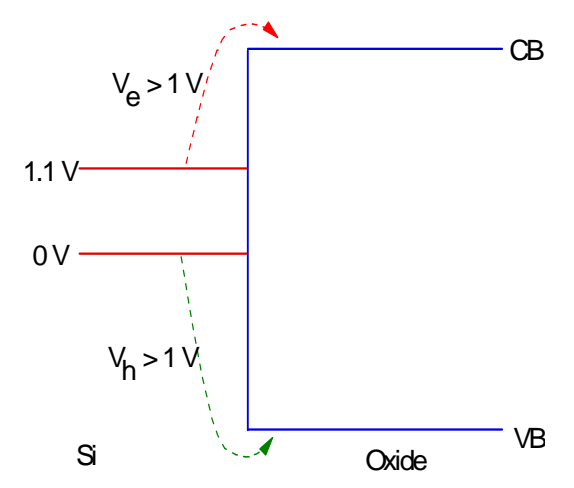

Fig 8

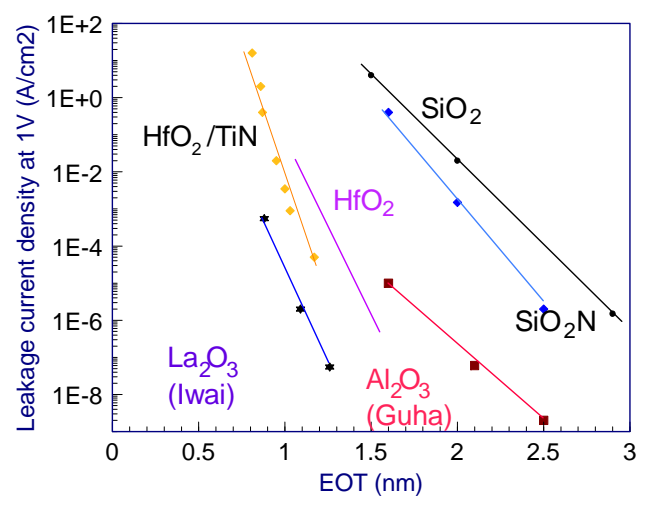

Fig 9 


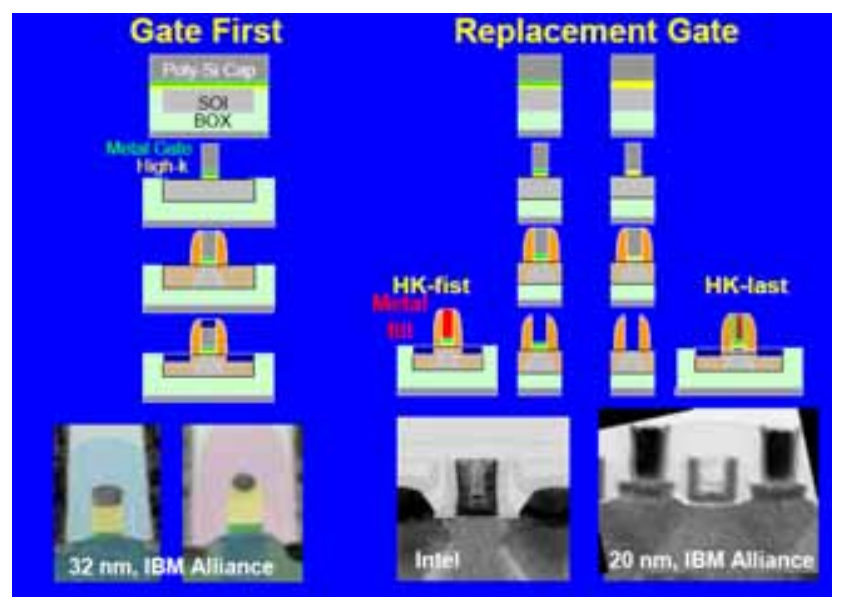

Fig 10.

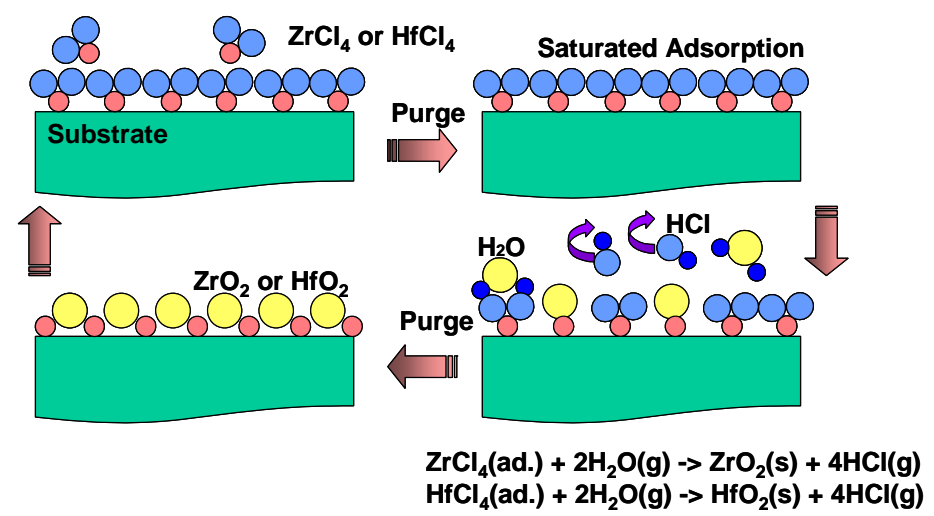

Fig 11

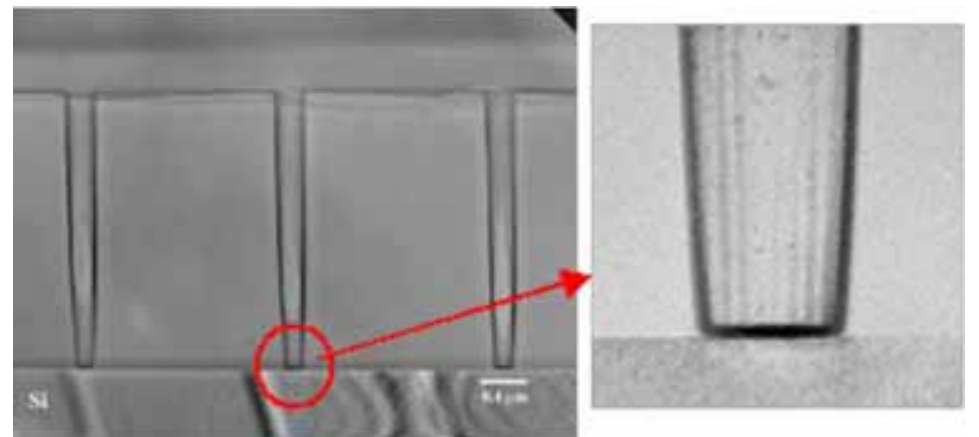

Fig 12 


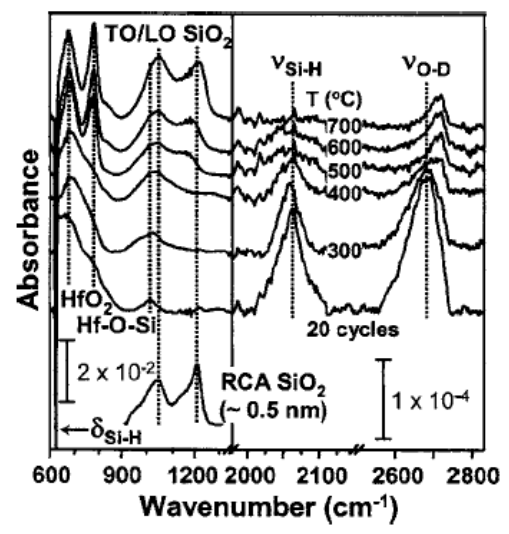

Fig 13
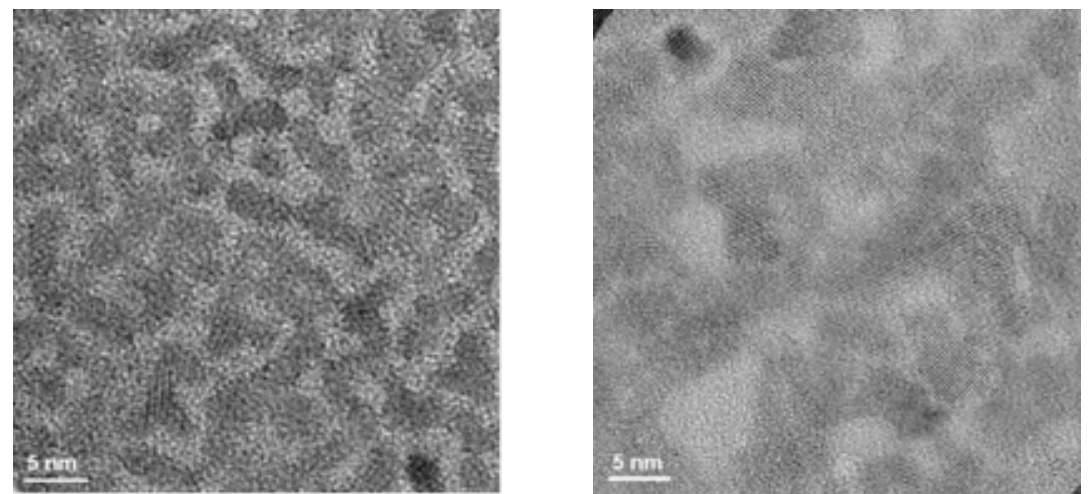

Fig 14
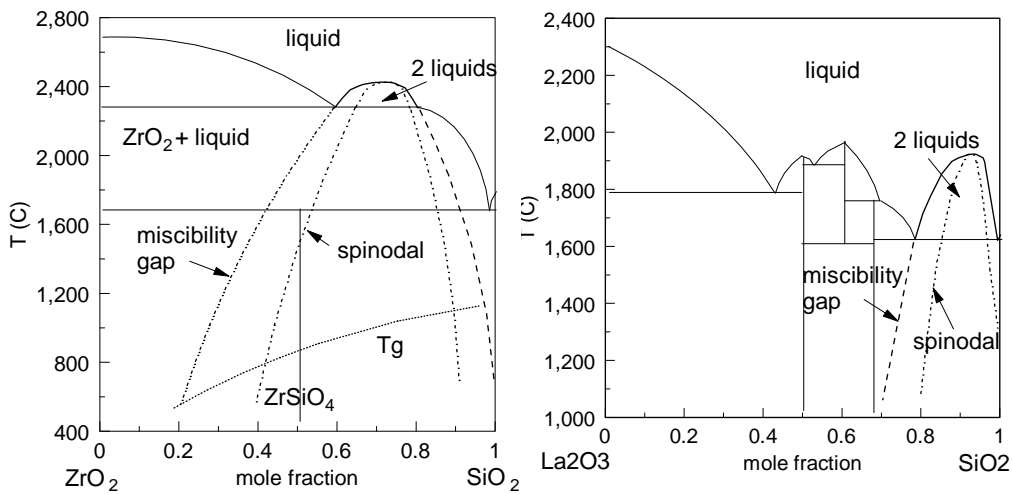

Fig 15 


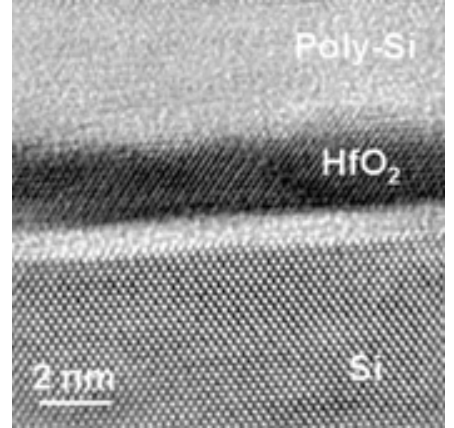

Fig 16
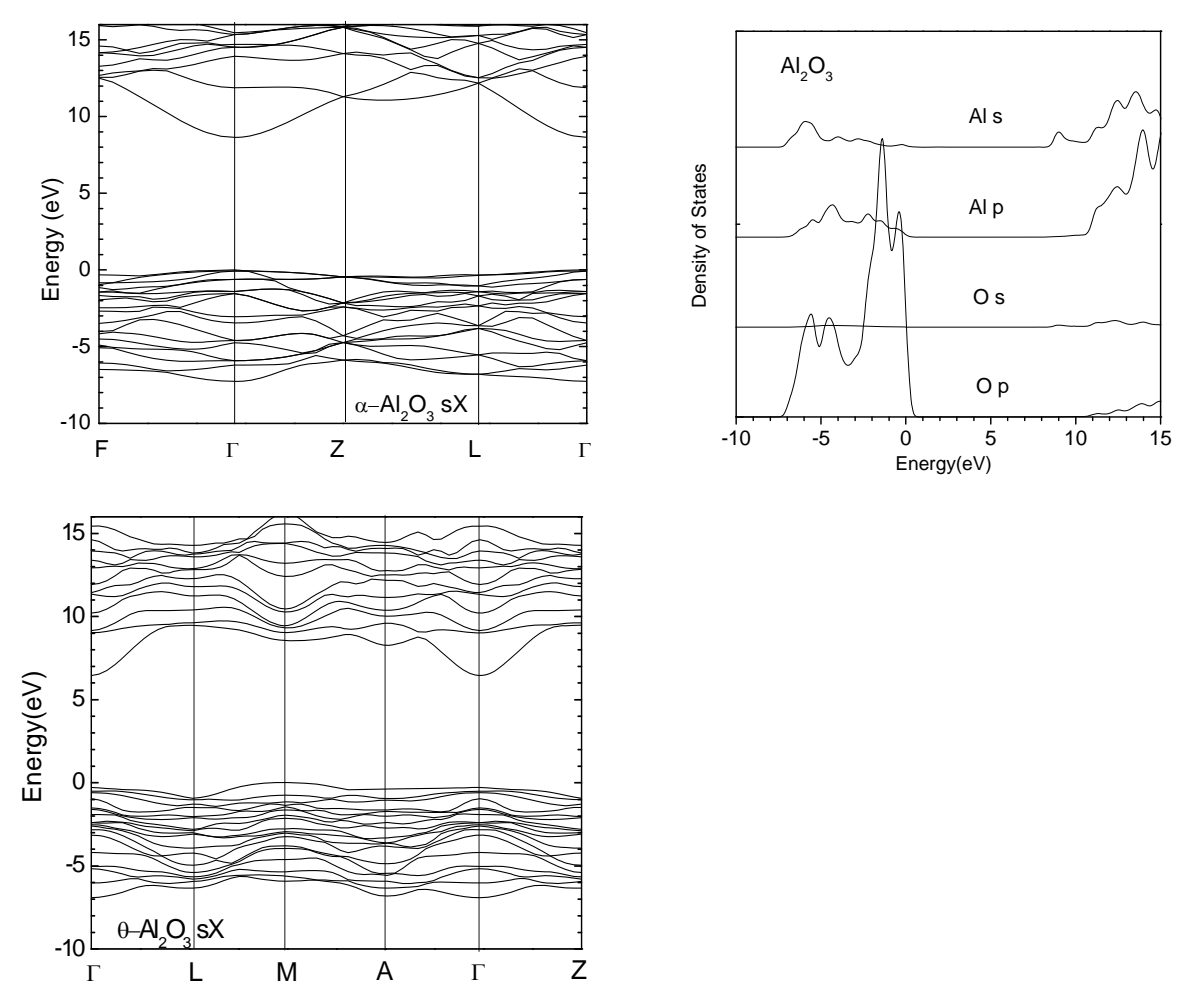

Fig 17
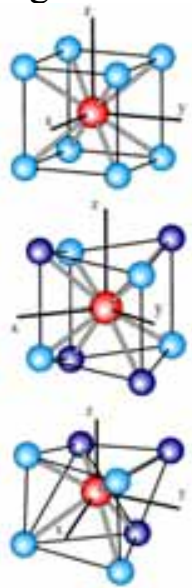

Fig. 18.

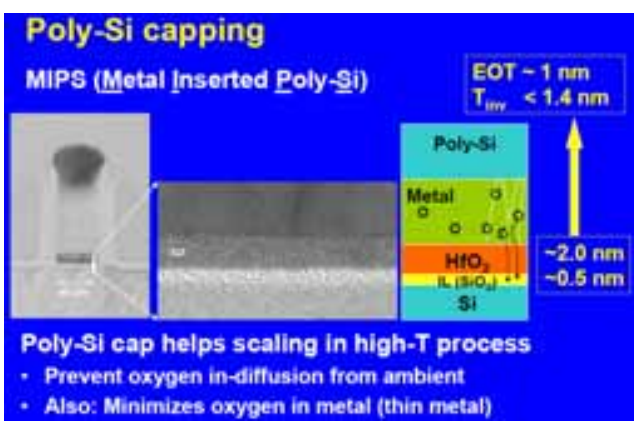

Also: Minimizes oxygen in metal (thin meta) 

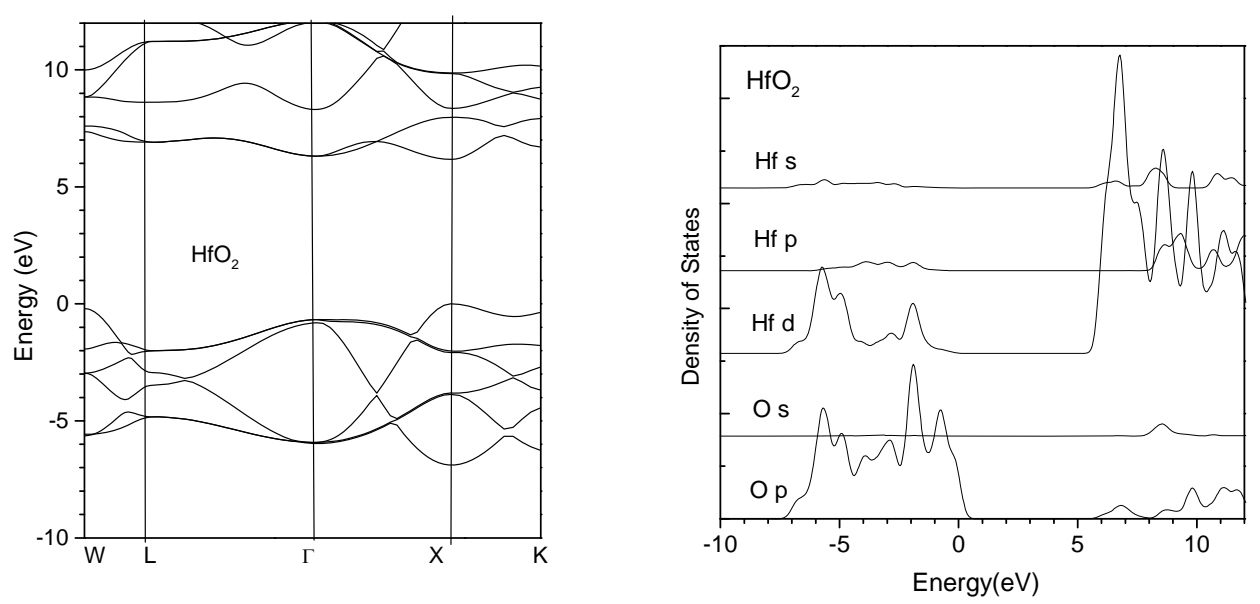

Fig. 19.
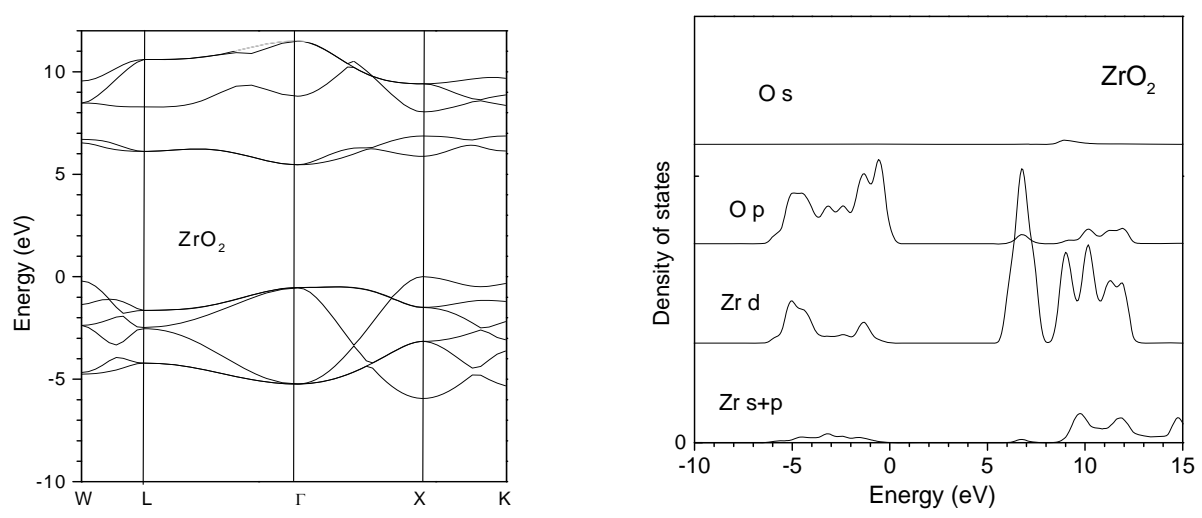

Fig 20

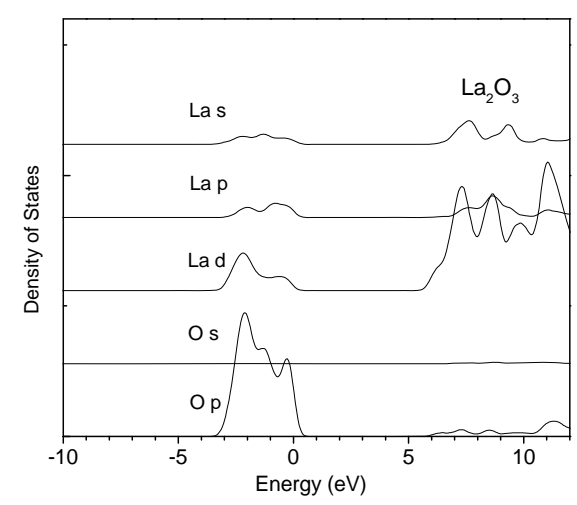

Fig 21 


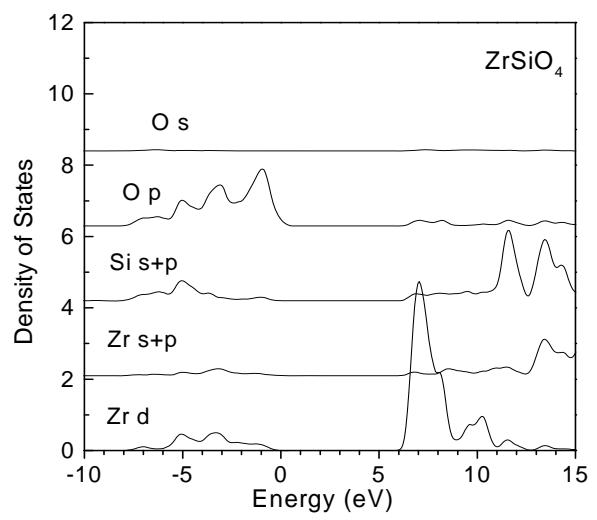

Fig 22
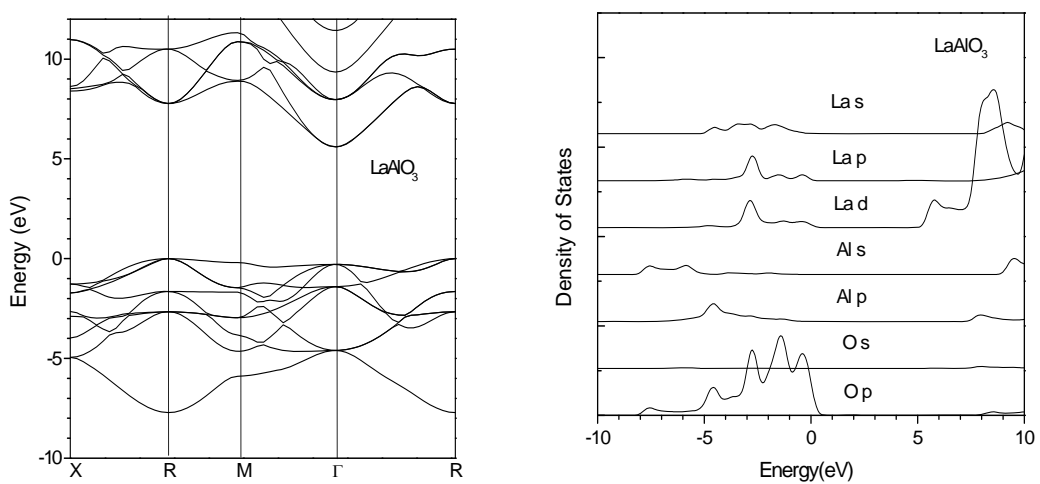

Fig 23

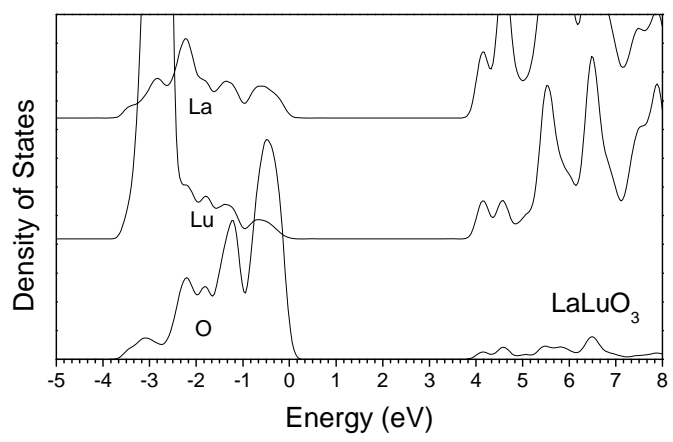

Fig 24 


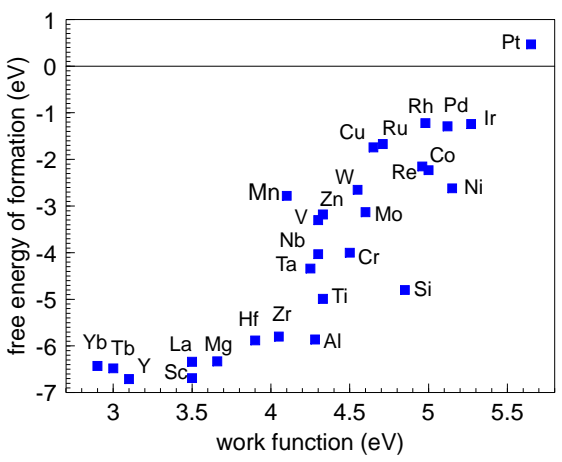

Fig 25.

Non-interacting interface No charge transfer. $\mathrm{S}=1$

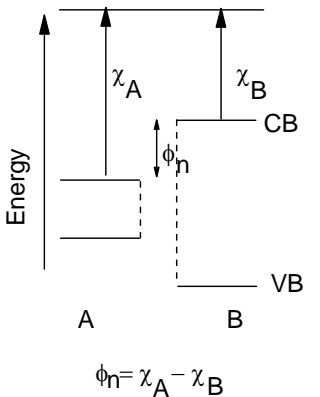

Pinned interface, dipoles Aligned CNLs. $\mathrm{S}=0$

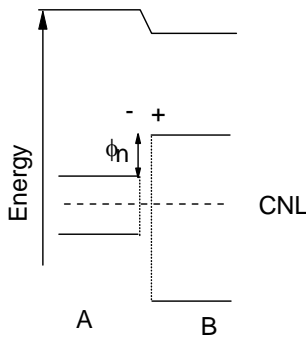

Fig 26
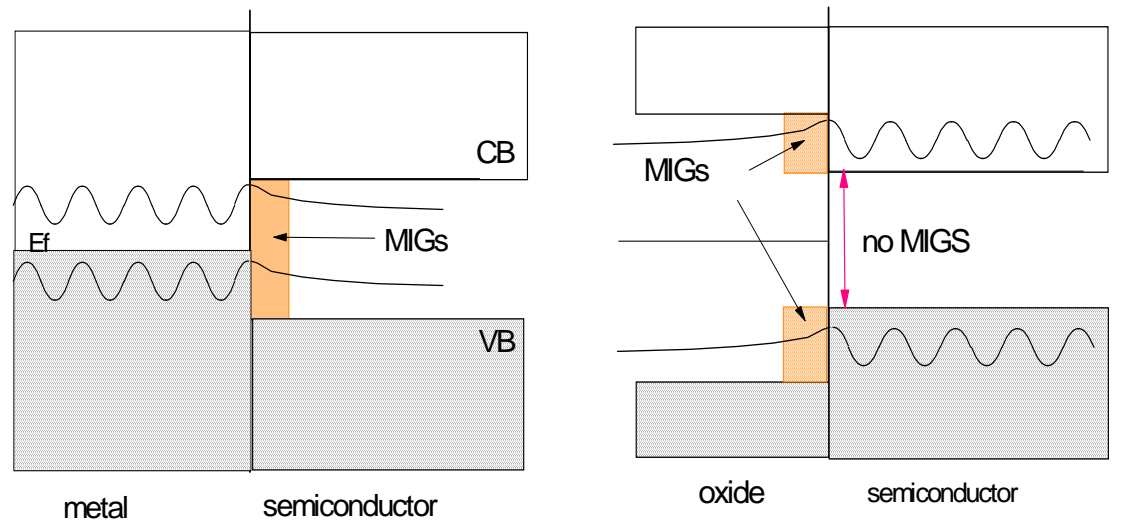

Fig. 27. 


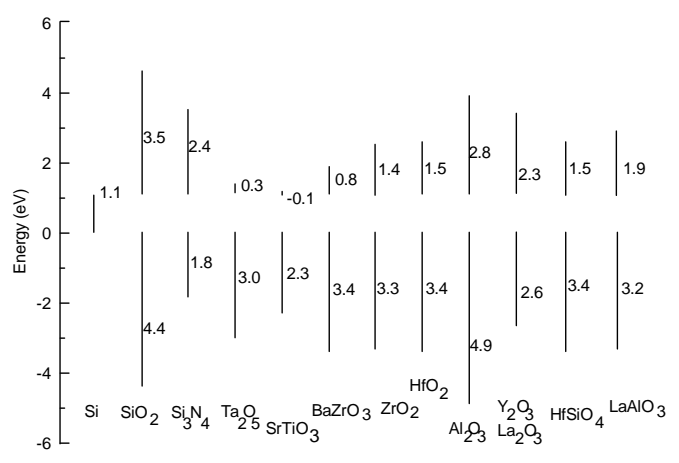

Fig 28

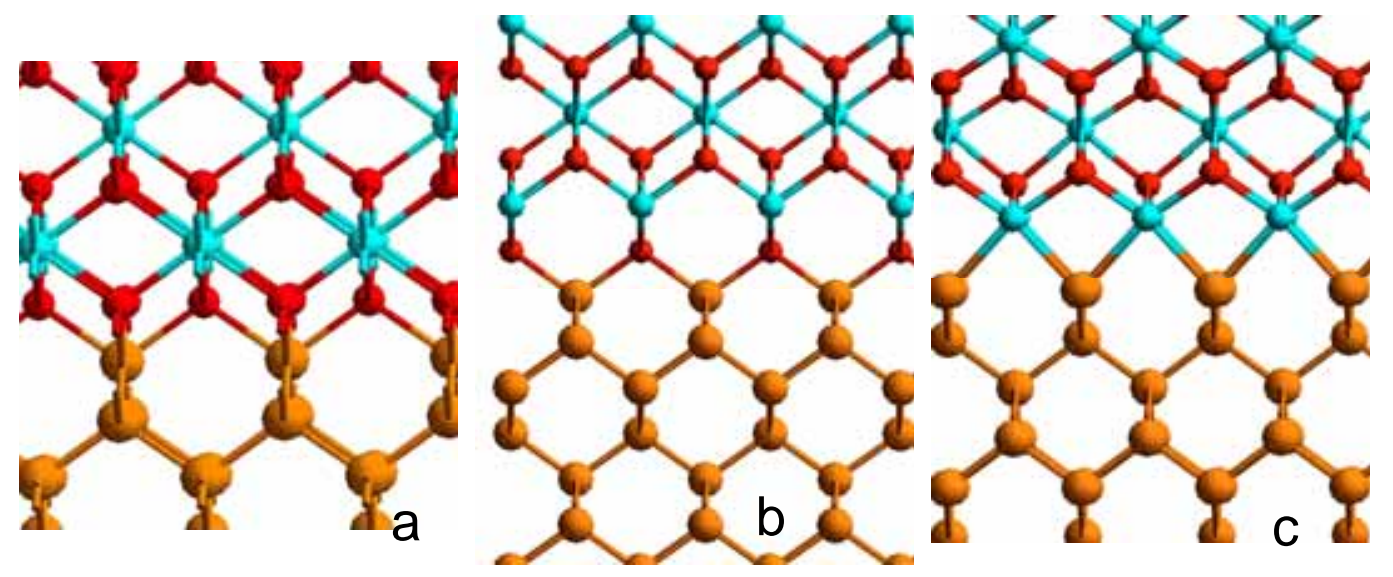

Fig. 29
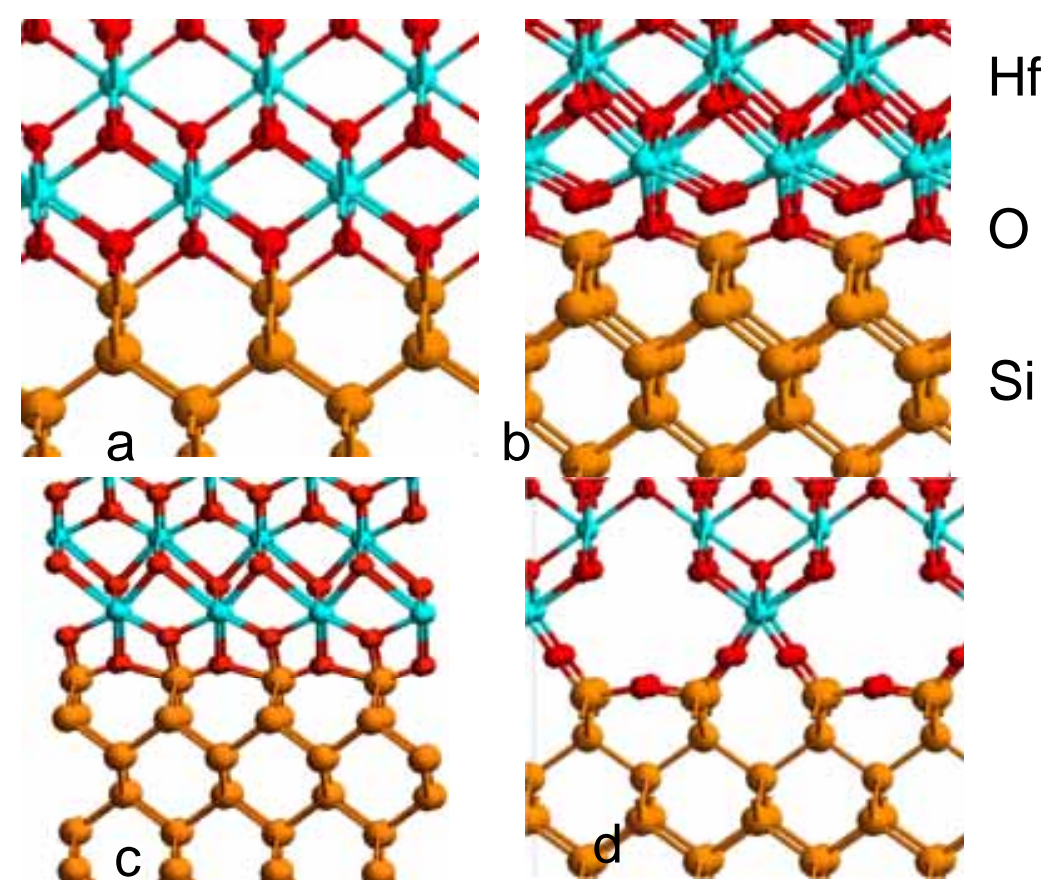

Fig. 30 


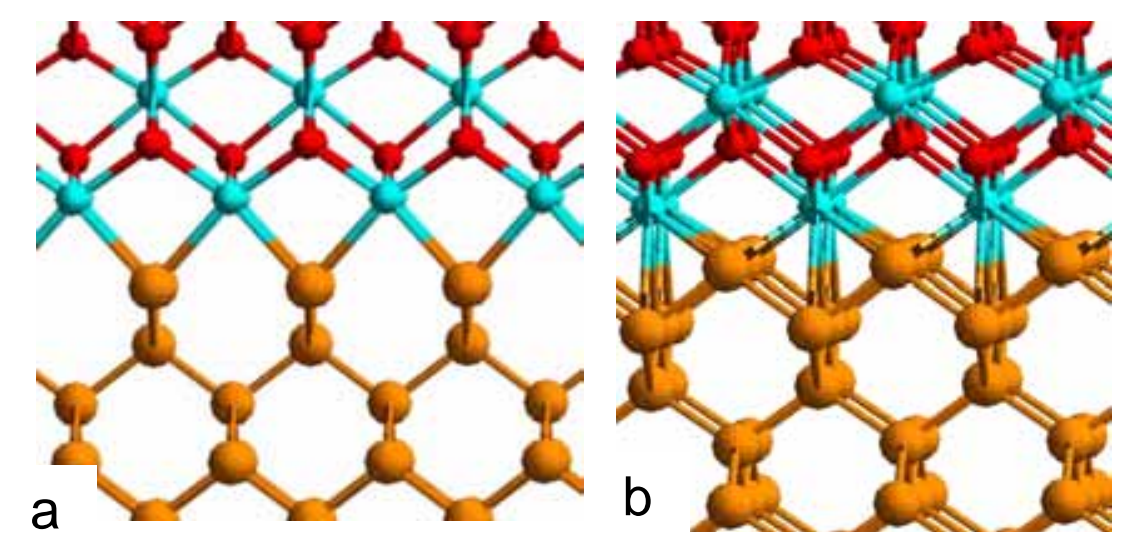

Fig. 31.
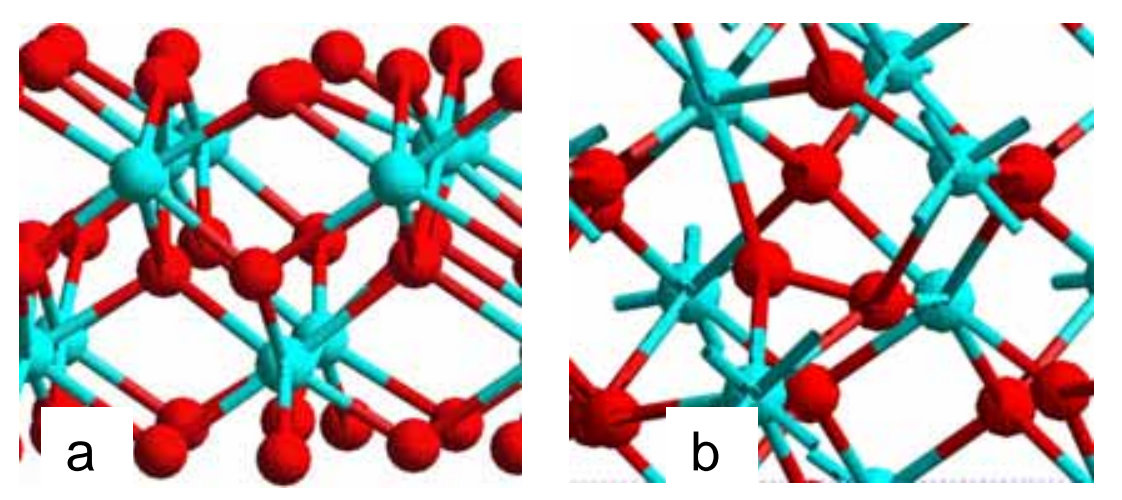

Fig 32

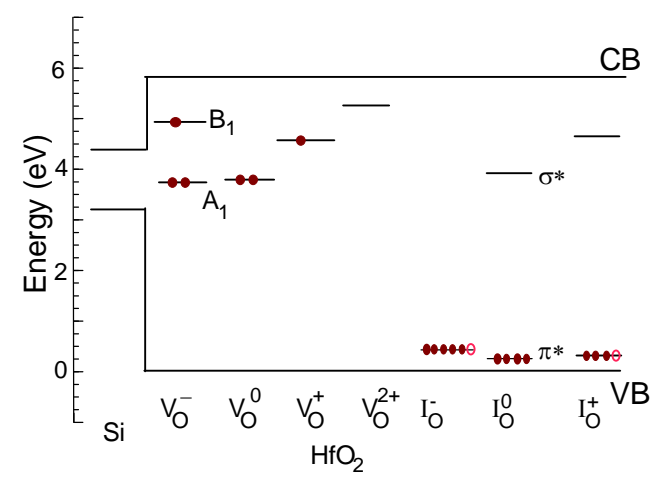

fig 33 

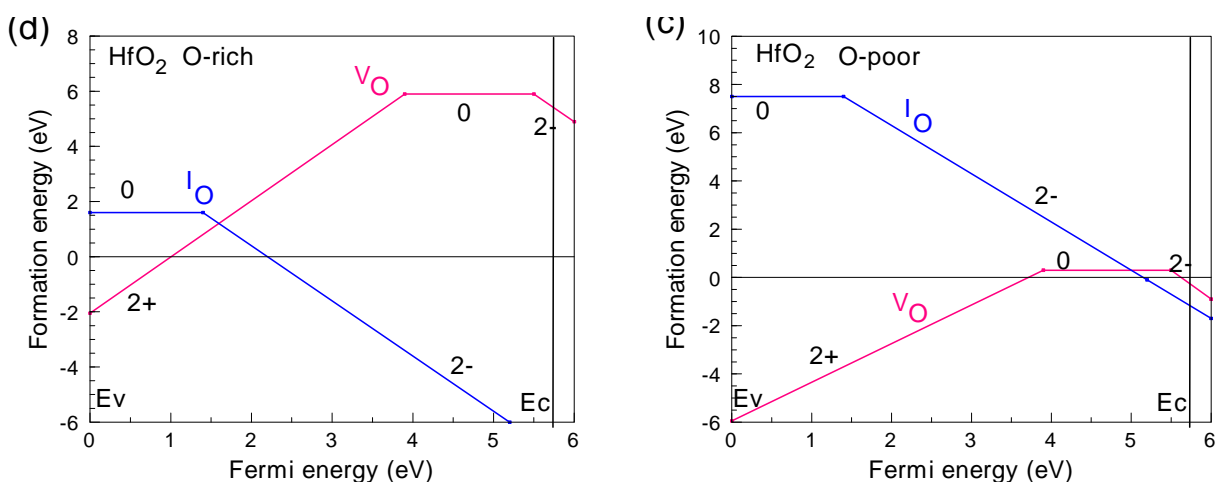

Fig 34 .

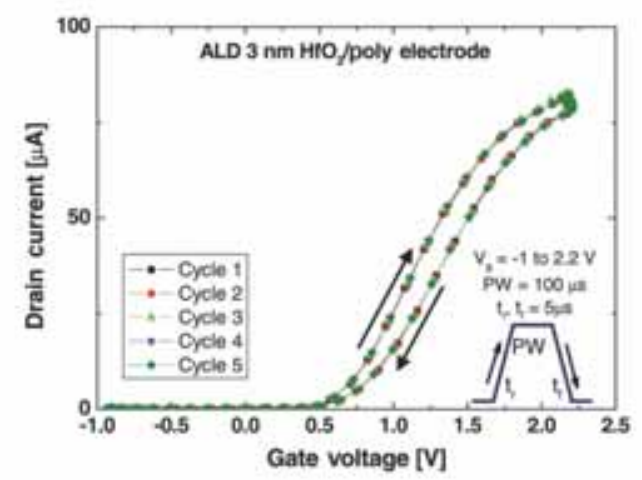

Fig. 35
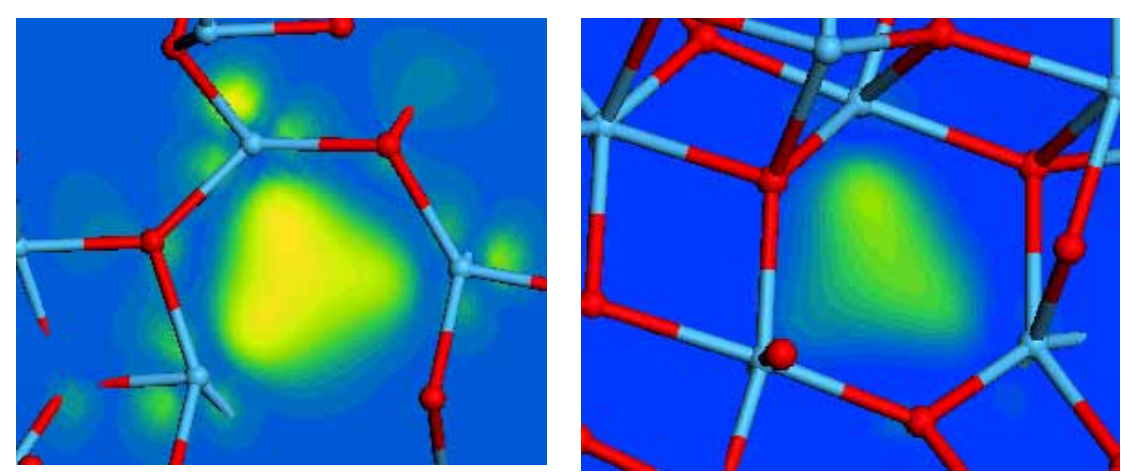

Fig 36 

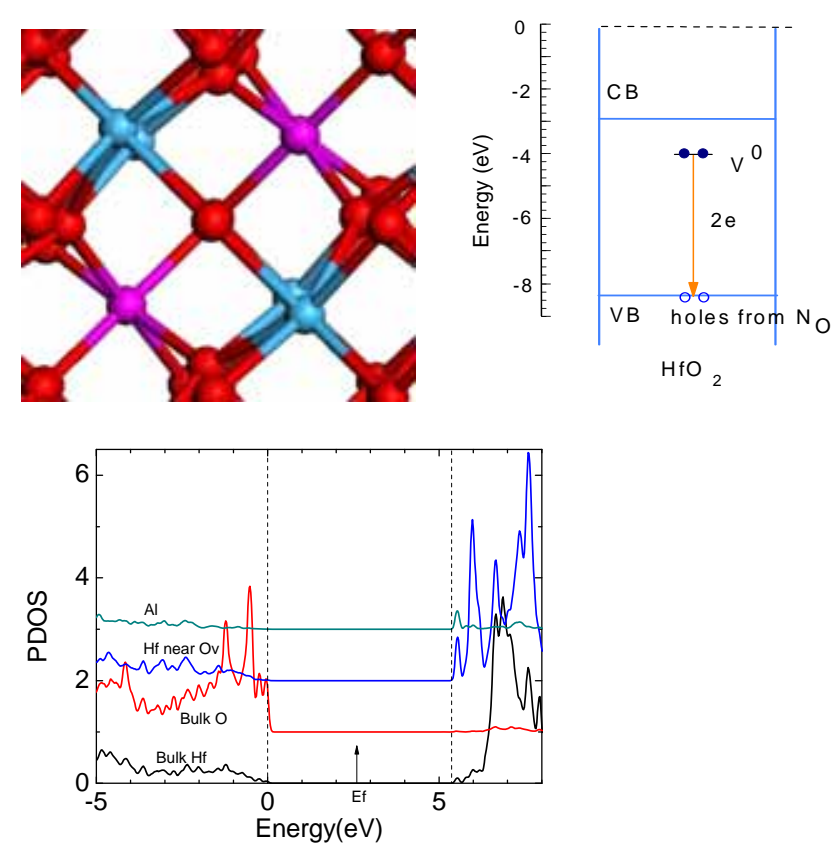

Fig. 37.

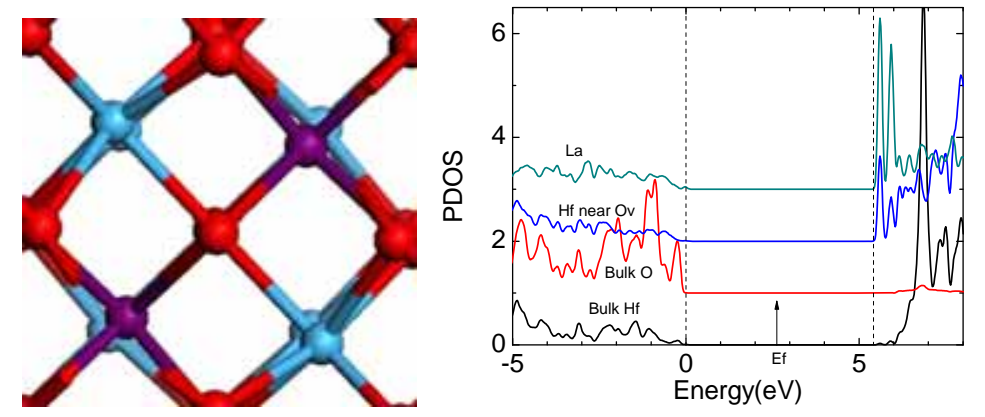

fig 38

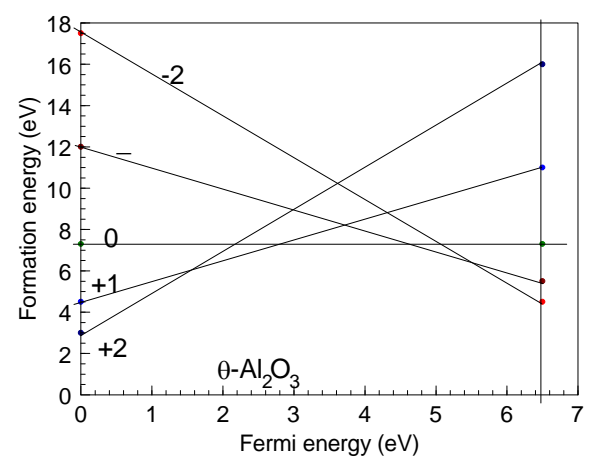

Fig. 39. 

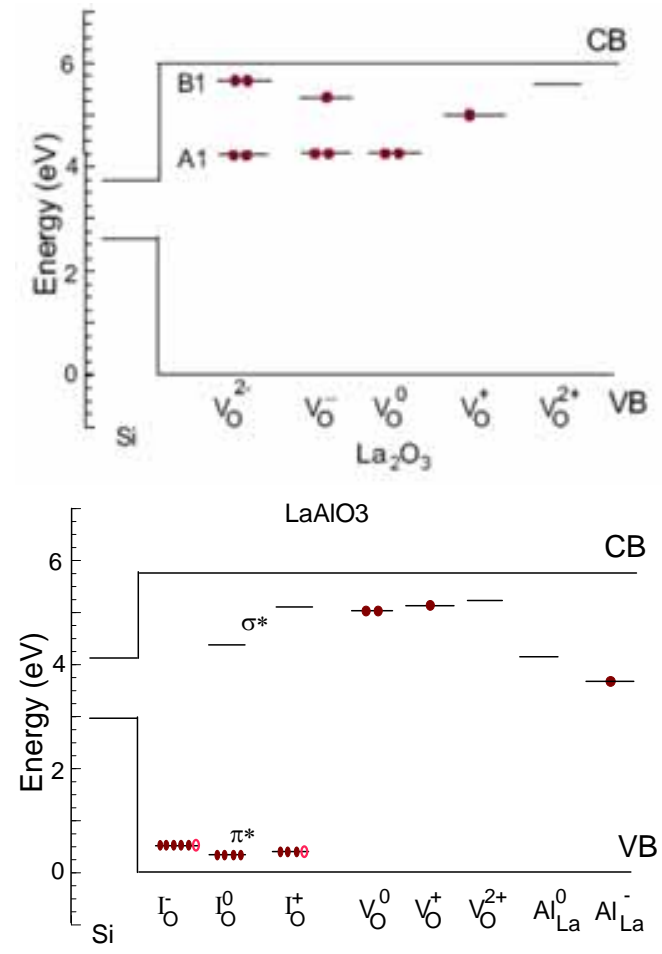

Fig 40

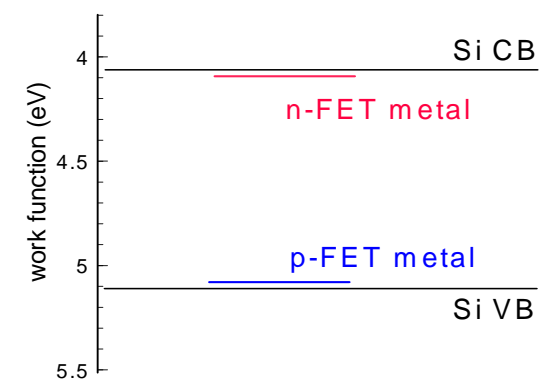

Fig 41

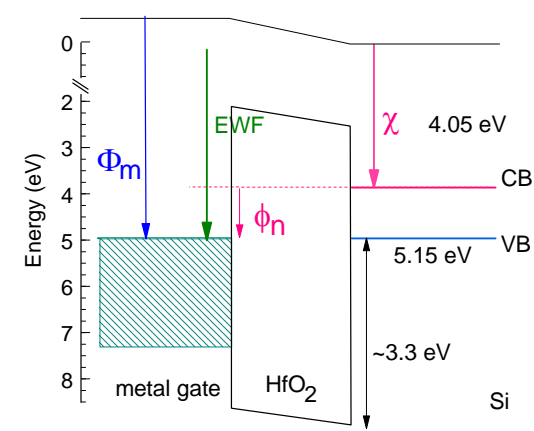

Fig 42 

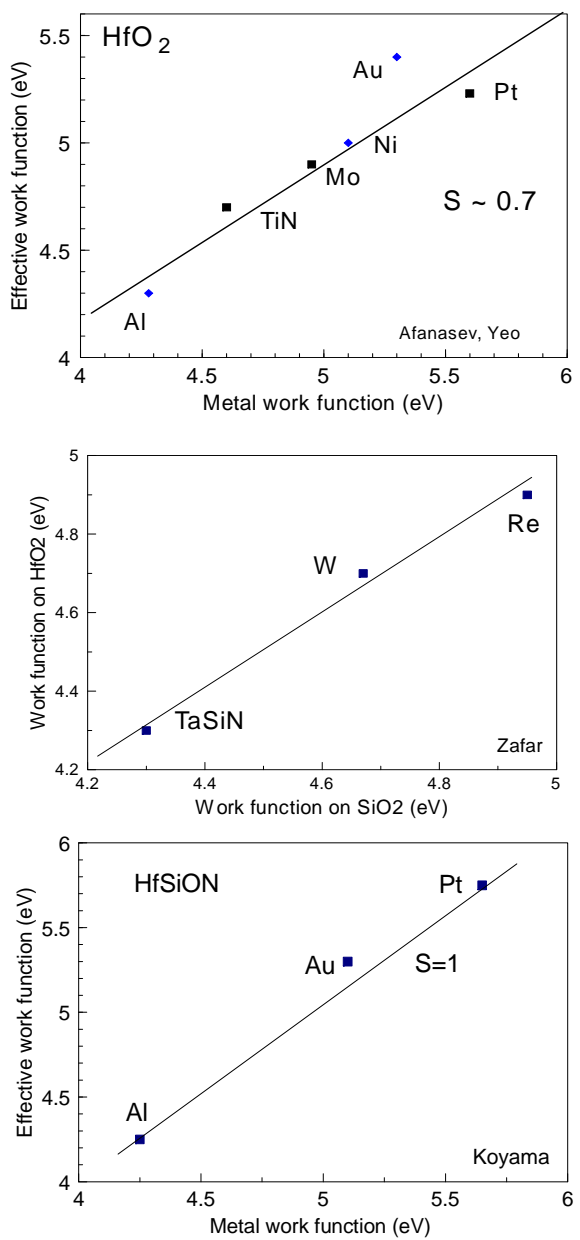

Fig 43.

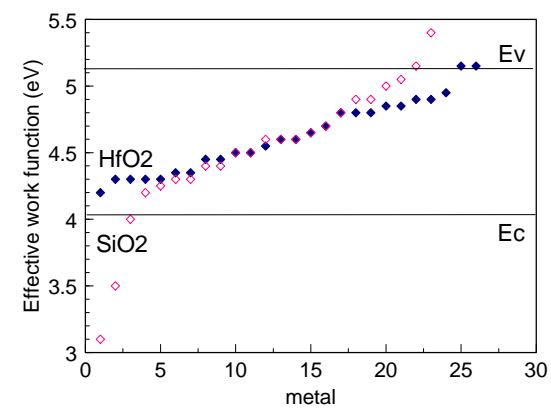

Fig. 44. 


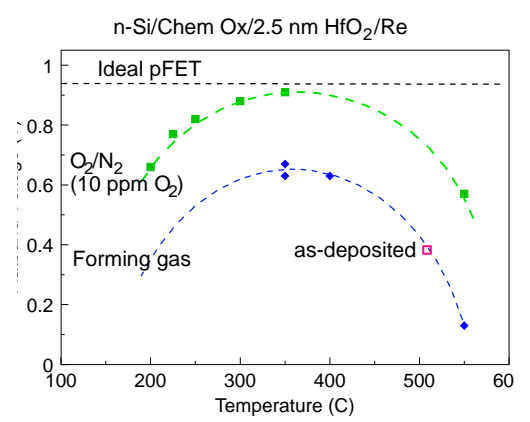

Fig. 45 .
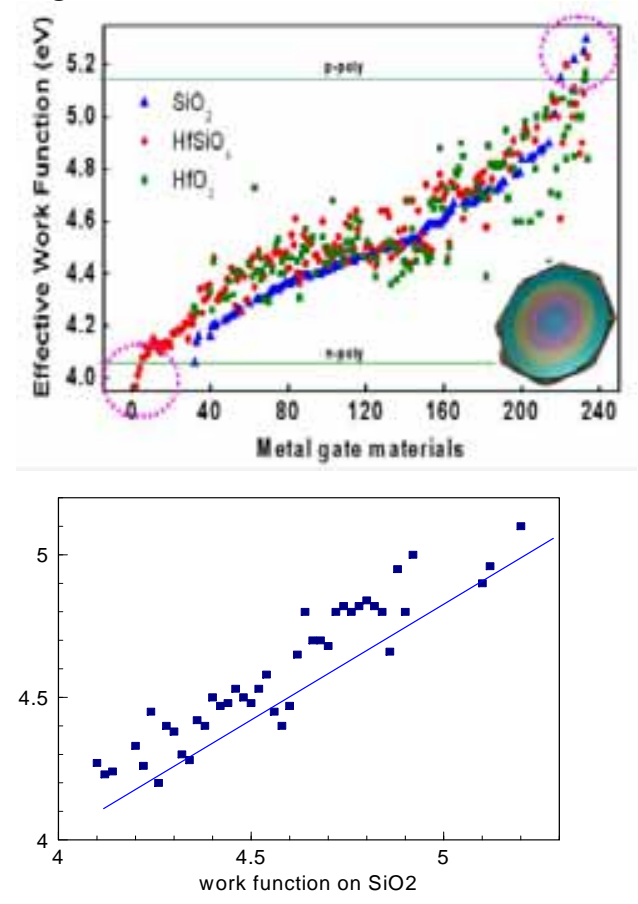

Fig. 46.

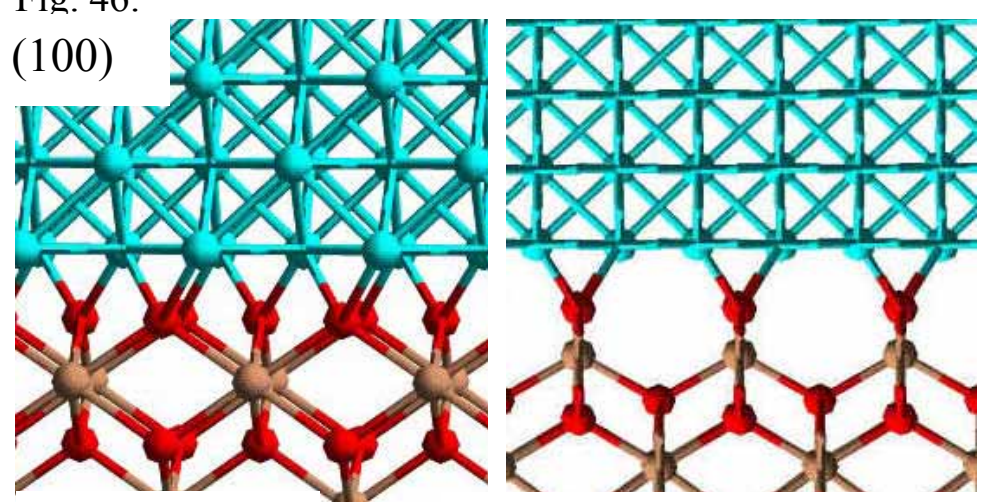

(a) O-rich (b)non-polar O-terminated

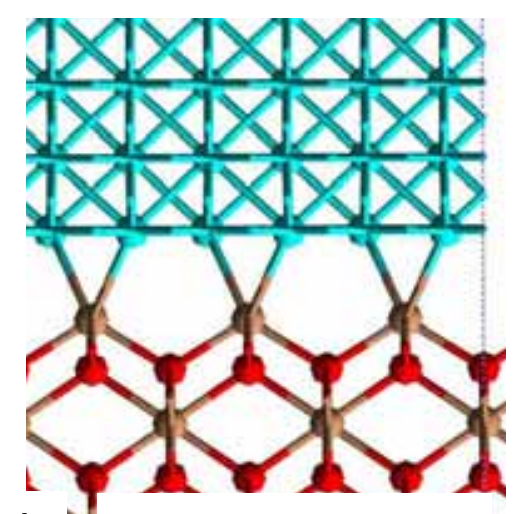

(c) Hf-rich

fig 47 


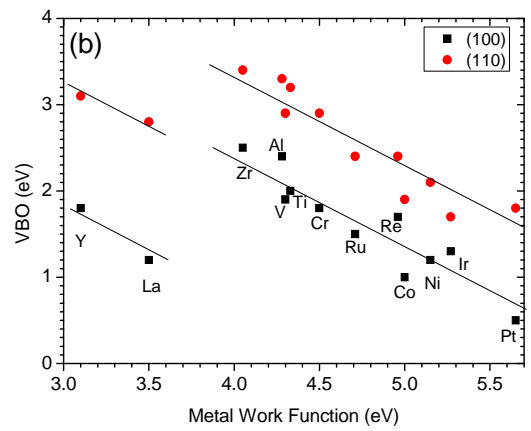

fig 48
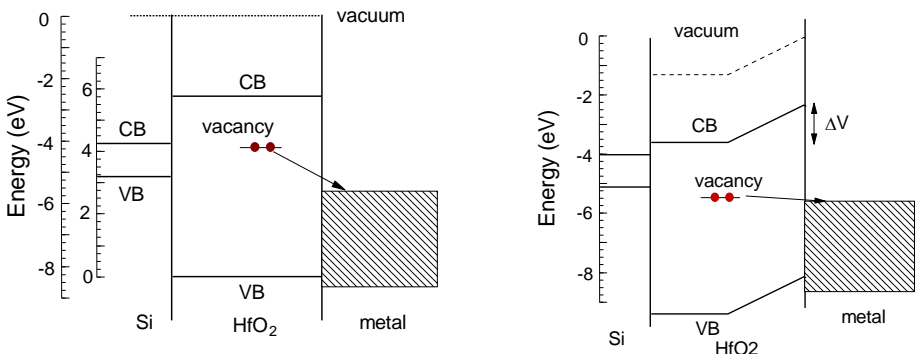

Fig. 49.

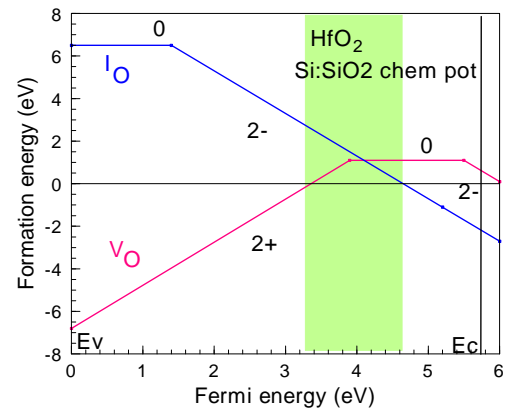

Fig 50

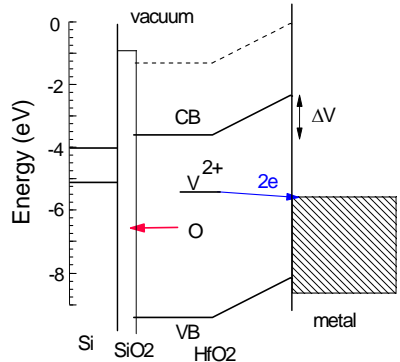

fig 51 


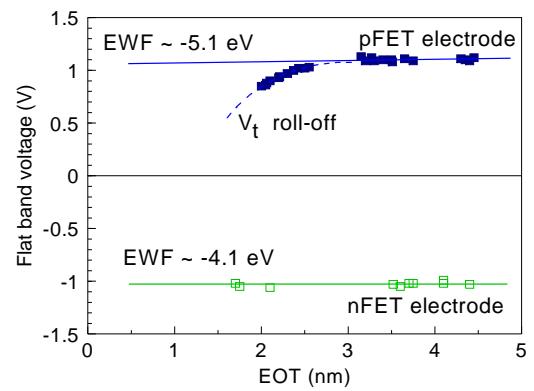

Fig 52

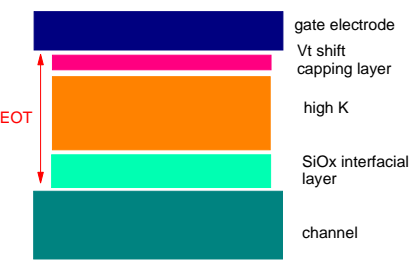

Fig 53

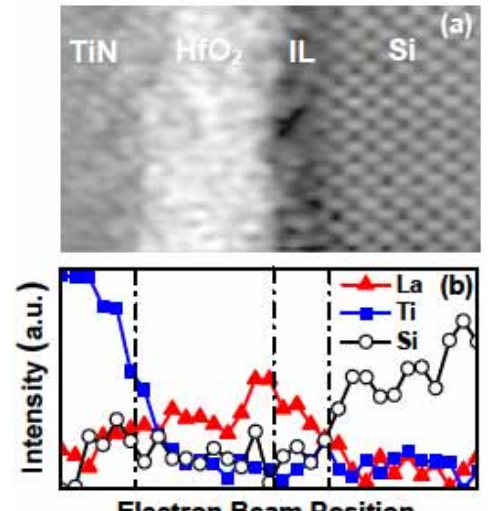

Fig 54

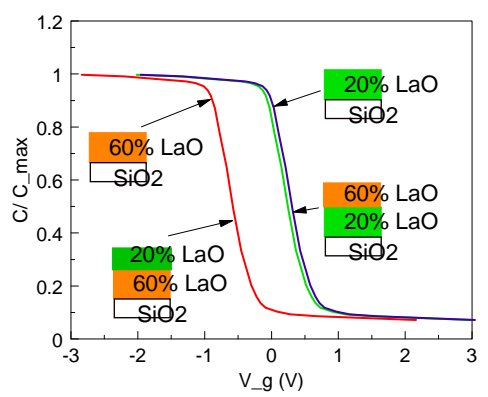

Fig 55 


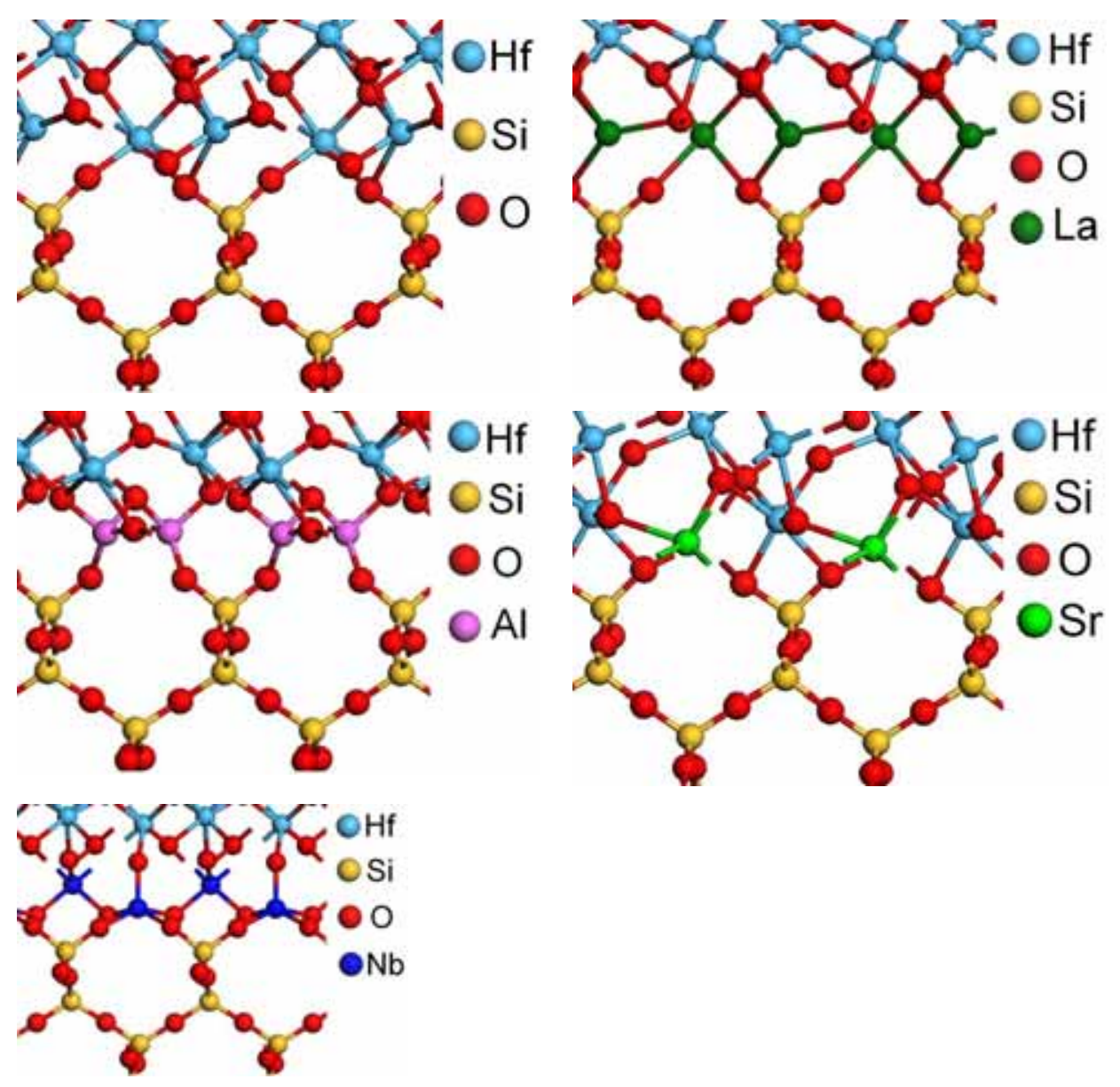

Fig 56 

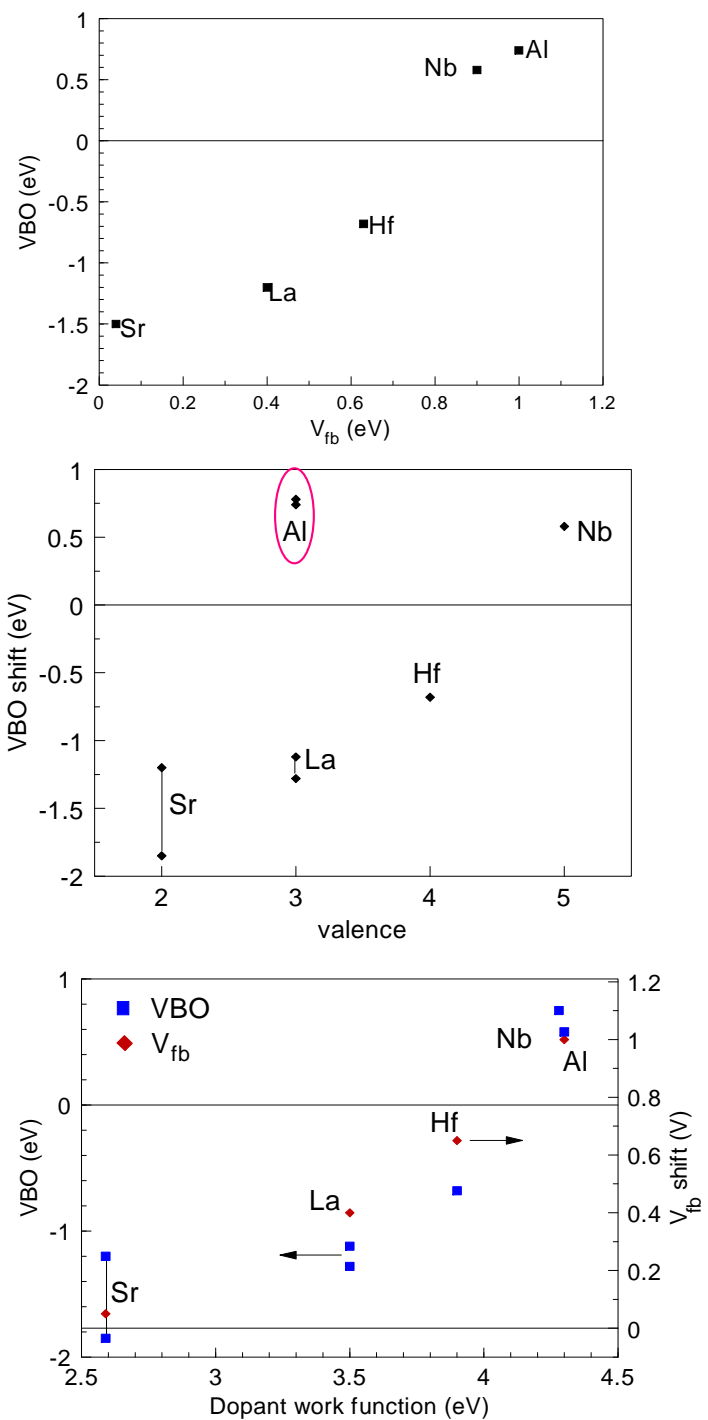

Fig 57

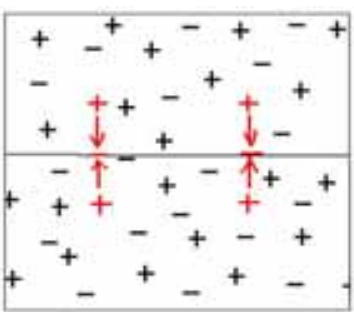

(a) no net dipole in amorphous phase

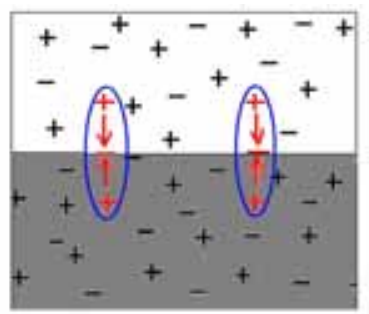

(b) net dipole builds up due to screening difference

Fig 58 


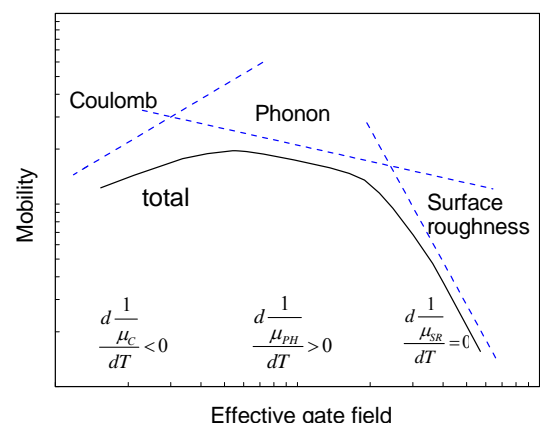

Fig 59
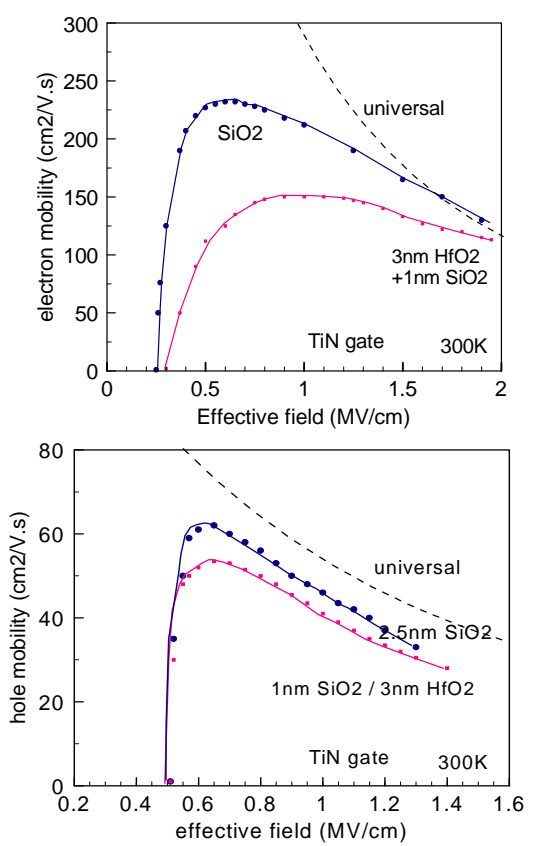

Fig 60.

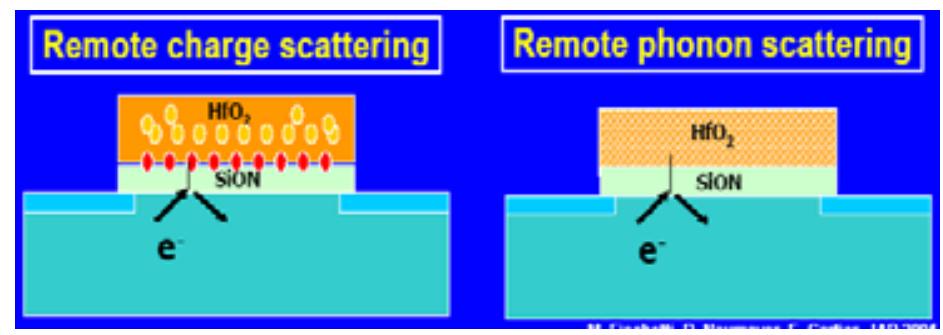

Fig 61 


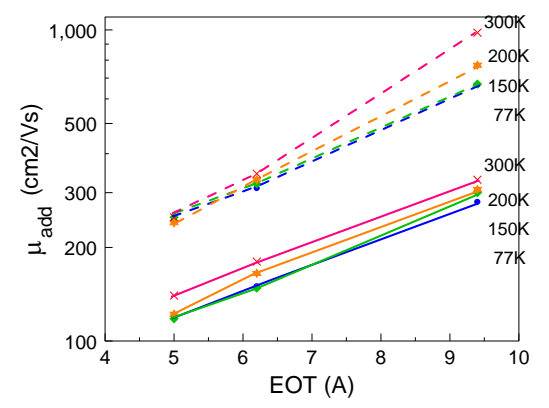

Fig 62.

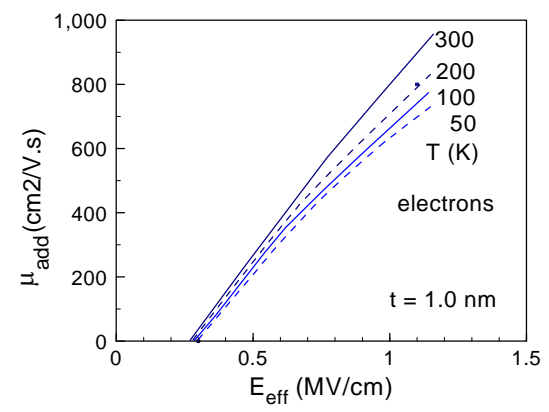

Fig 63

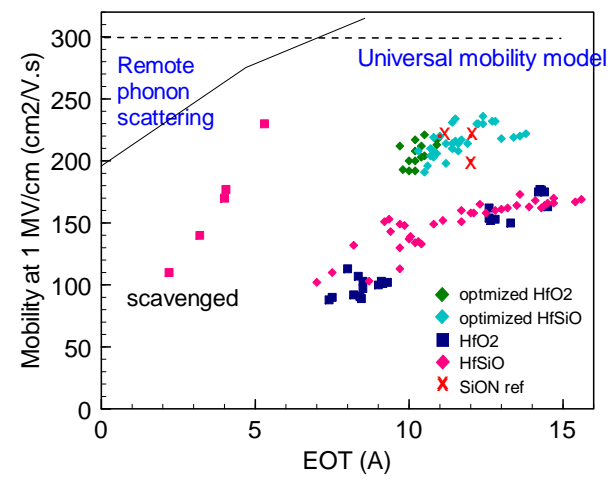

Fig 64

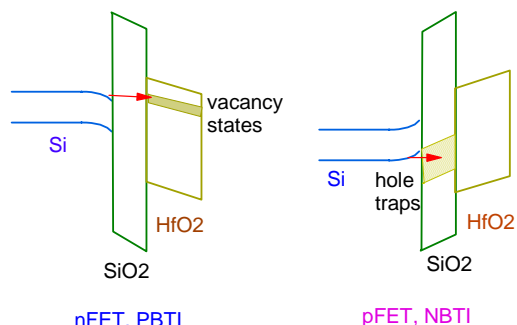

Fig 65. 


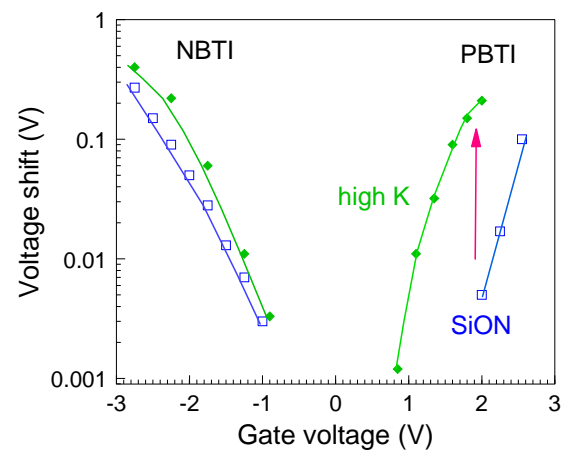

Fig. 66

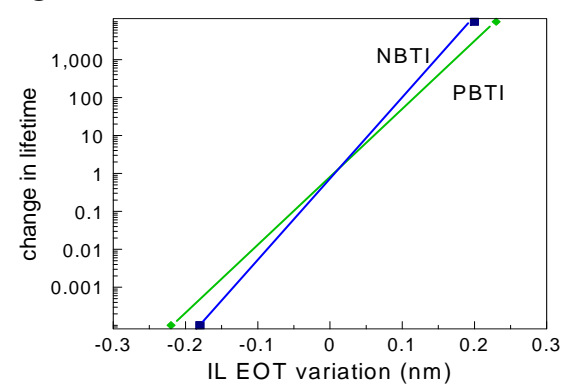

Fig. 67

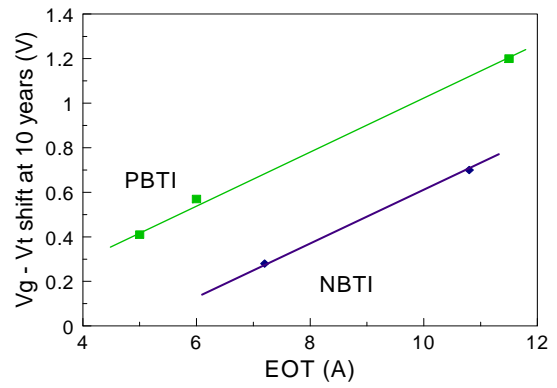

Fig 68.

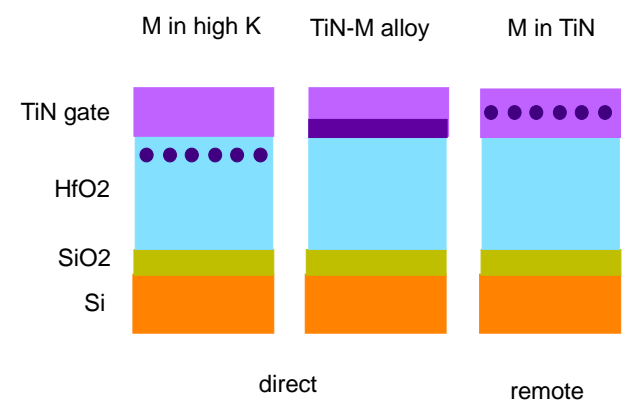

Fig 69. 


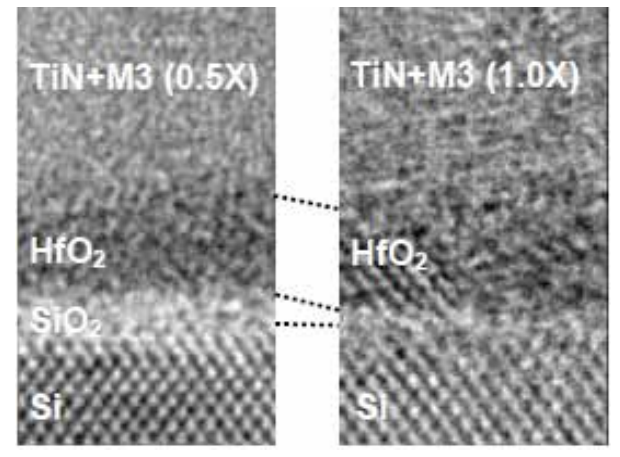

Fig 70

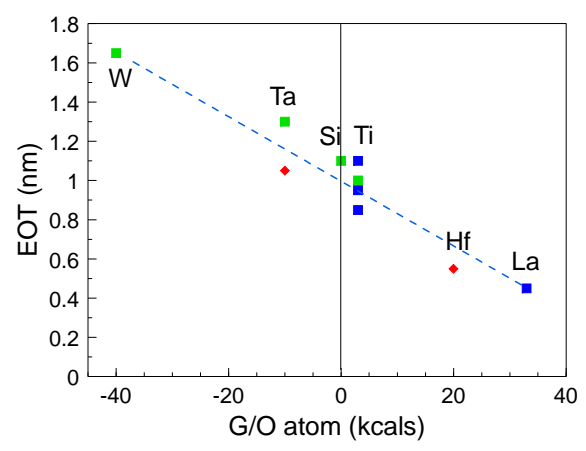

Fig 71.

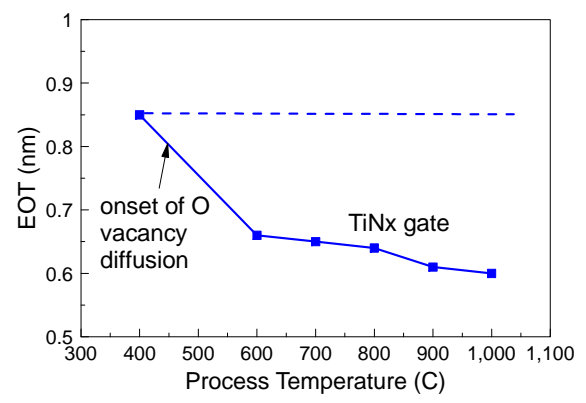

Fig. 72 .

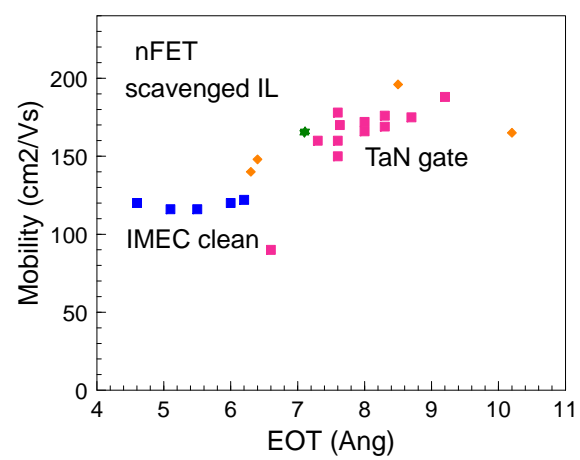

fig 73 


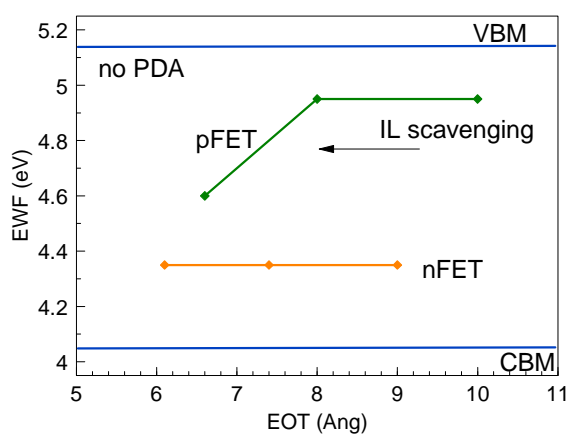

Fig 74

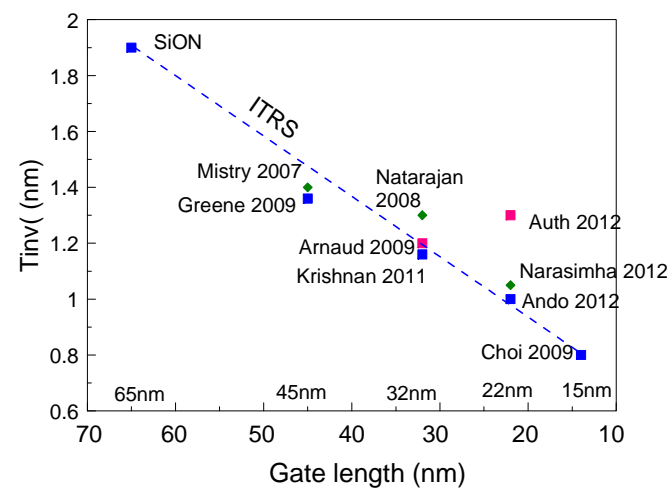

Fig 75

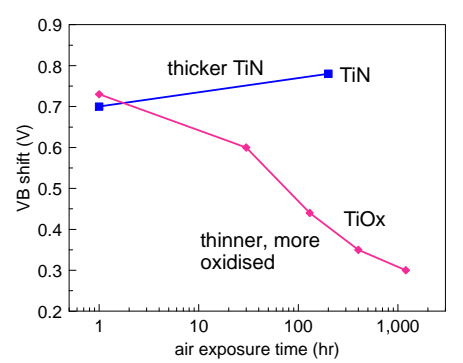

Fig 76.
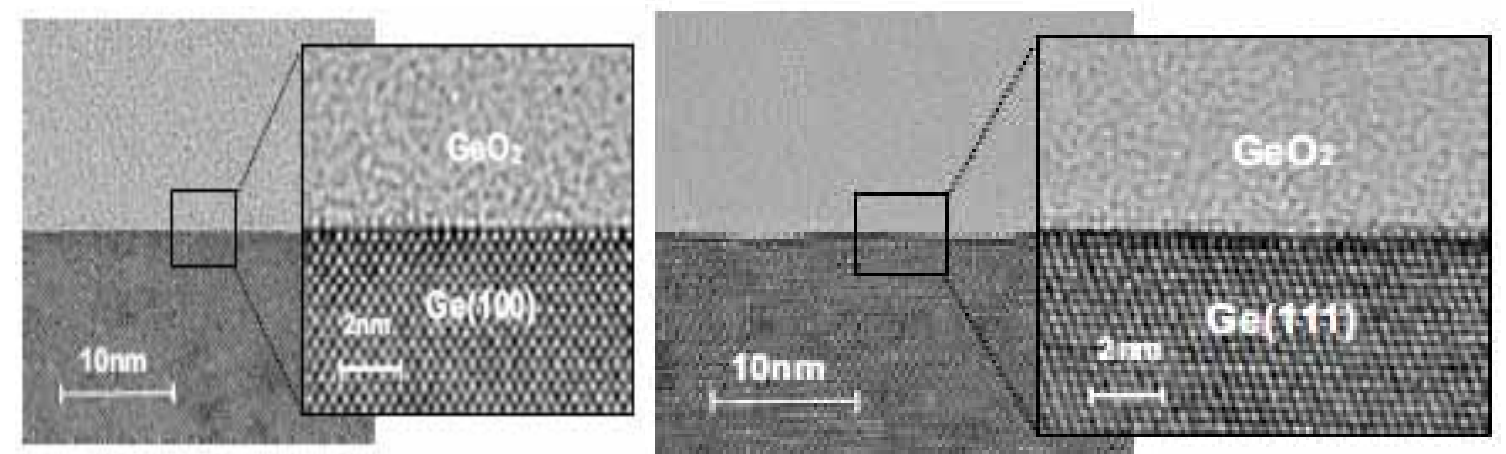

Fig 77. 


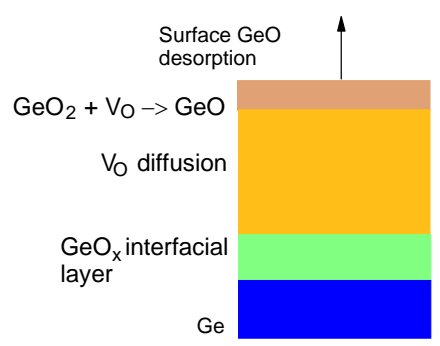

Fig 78.
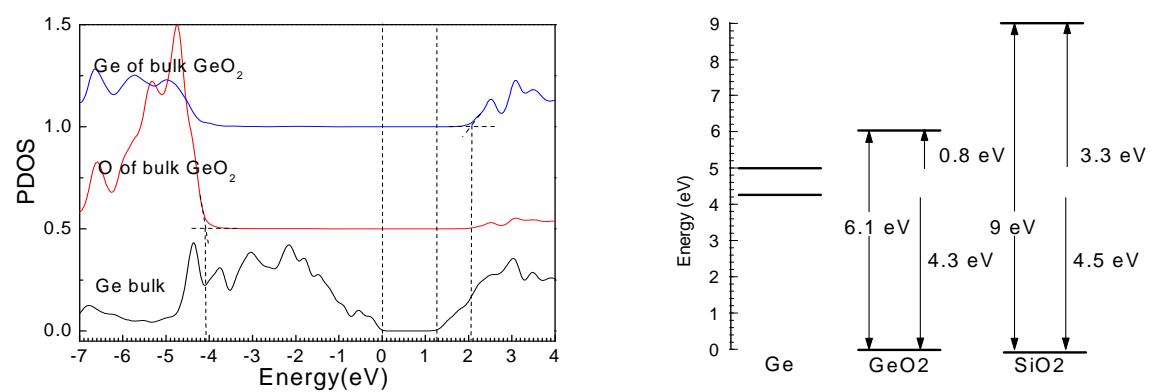

Fig 79

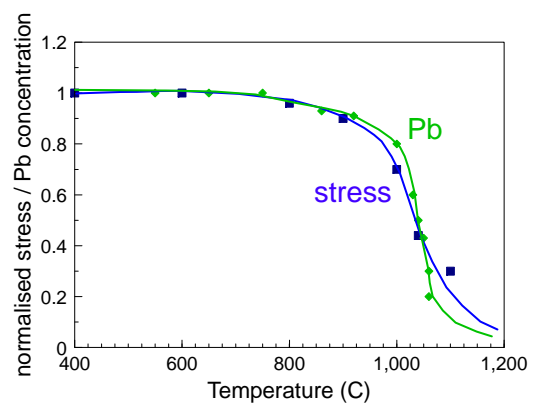

Fig 80

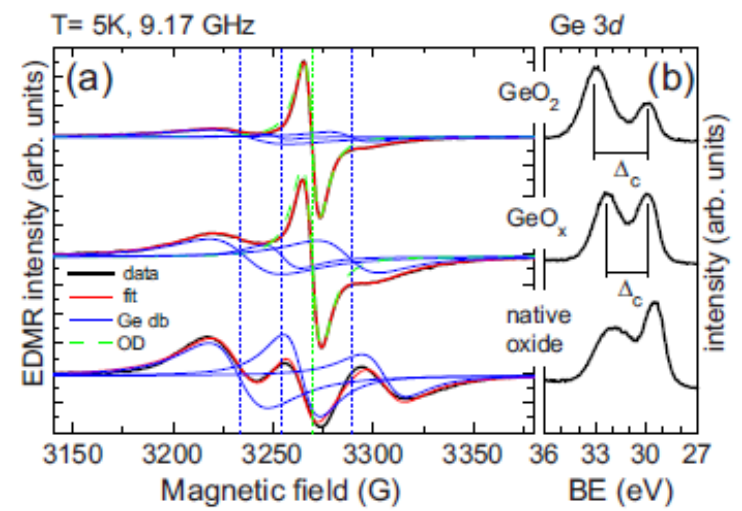

Fig 81 

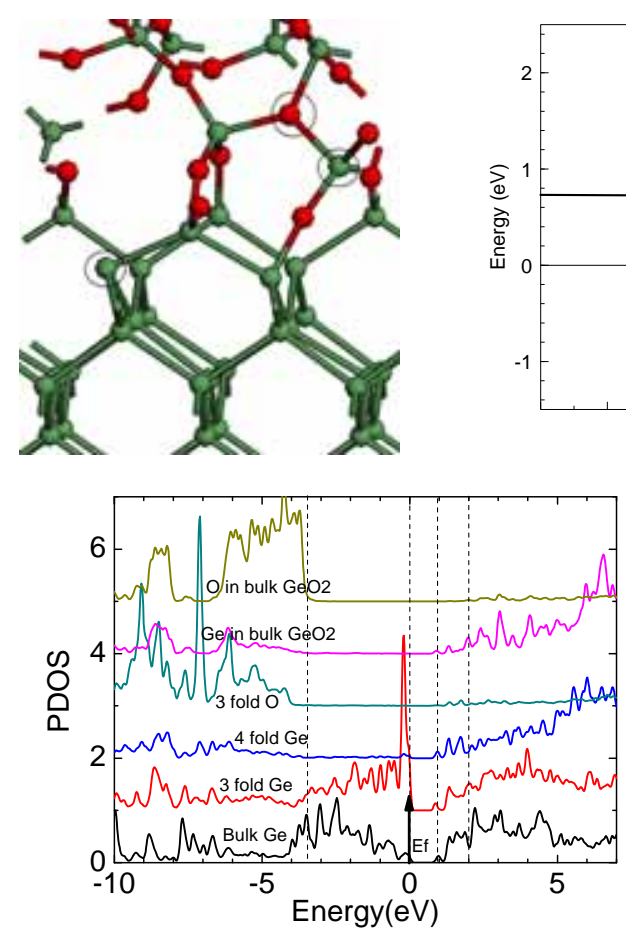

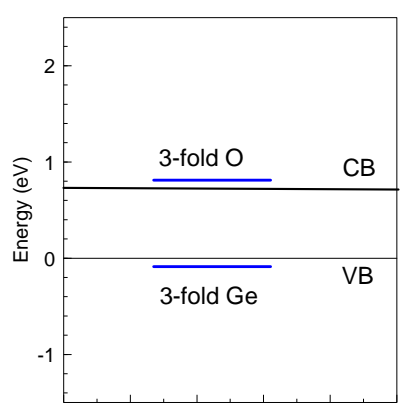

Fig 82.
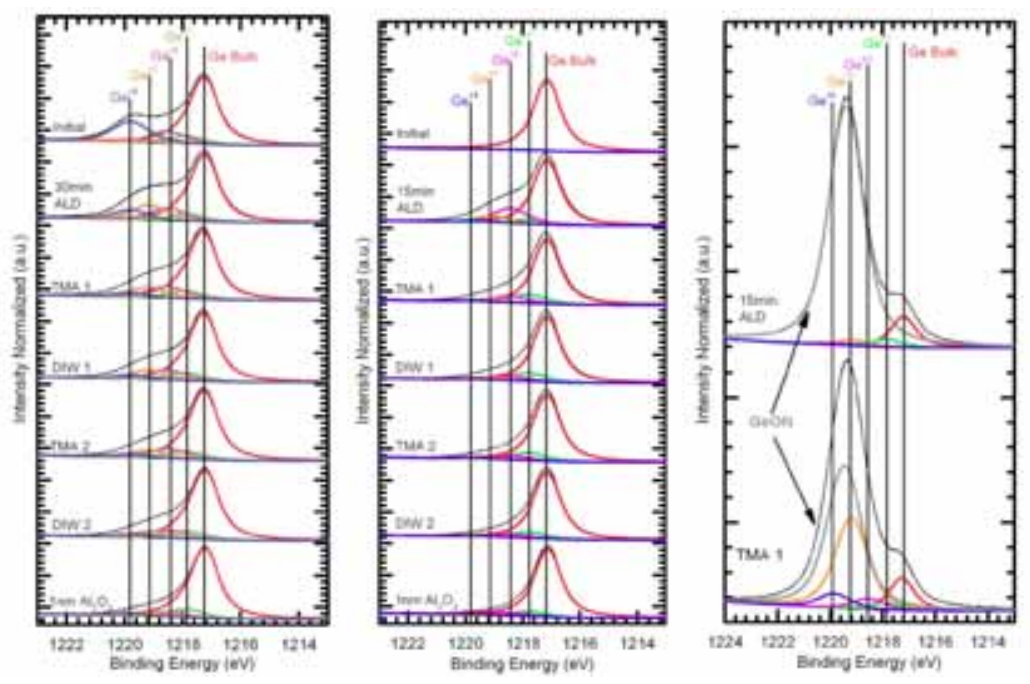

Fig 83.

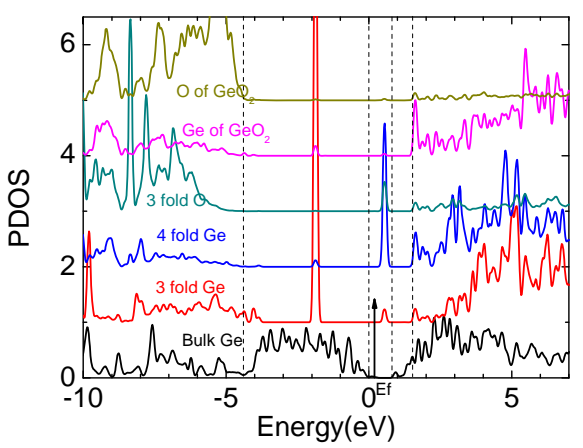




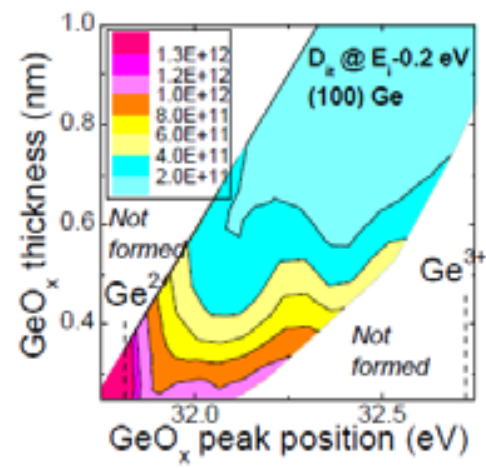

Fig 84

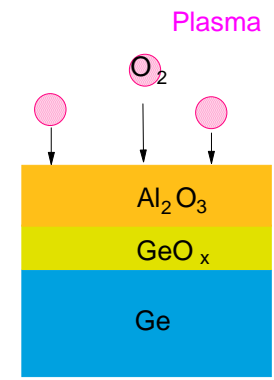

Fig 85

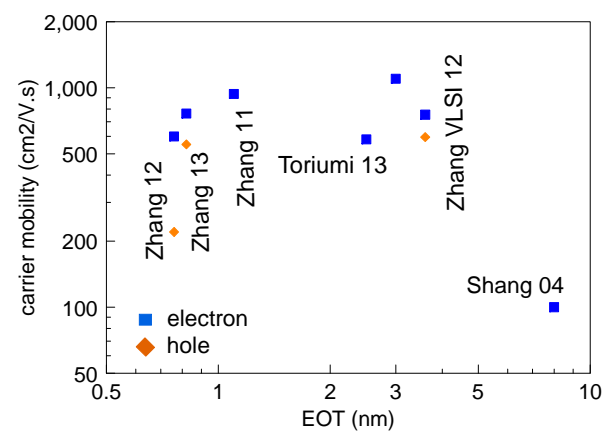

Fig 86 